Environmental Restoration Division Portsmouth Environmental Restoration Program

\title{
Applicable or Relevant and Appropriate Requirements (ARARs) for Remedial Actions at the Paducah Gaseous Diffusicn Plant A Compendium of Environmental Laws and Guidance
}

E. L. Etnier

L. A. Eaton

Date Issued-March 1992

Prepared by

Health and Safety Research Division

Oak Ridge National Laboratory

Prepared for

U.S. Department of Energy

Office of Environmental Restoration and Waste Management under budget and reporting code EW 20

PADUCAH GASEOUS DIFFUSION PLANT

Paducah, Kentucky 42001 managed by MARTIN MARIETTA ENERGY SYSTEMS, INC.

for the

U.S. DEPAR'TMENT OF ENERGY

under contract DE-AC05-84OR21400

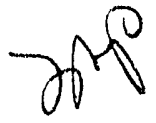

DISTAIBUTION OF THIS DOCUMENT IS UNLIMITEN 


\section{CONTENTS}

TABLES $\ldots \ldots \ldots \ldots \ldots \ldots \ldots \ldots \ldots \ldots \ldots \ldots \ldots \ldots \ldots \ldots$

ACRONYMS $\ldots \ldots \ldots \ldots \ldots \ldots \ldots \ldots \ldots \ldots \ldots \ldots \ldots \ldots \ldots \ldots$

ACKNOWLEDGMENTS $\ldots \ldots \ldots \ldots \ldots \ldots \ldots \ldots \ldots \ldots \ldots \ldots$ vii

EXECUTTVE SUMMARY $\ldots \ldots \ldots \ldots \ldots \ldots \ldots \ldots \ldots \ldots \ldots \ldots$ ix

1. INTRODUCTION $\ldots \ldots \ldots \ldots \ldots \ldots \ldots \ldots \ldots \ldots \ldots \ldots \ldots \ldots \ldots$

2. CHEMICAL-SPECIFIC ARARs ................... 4

2.1 GROUNDWATER AND SURFACE WATER $\ldots \ldots \ldots \ldots \ldots \ldots \ldots \ldots$

2.1 .1 Resource Conservation and Recovery Act ............... 4

2.1.2 Safe Drinking Water Act $\ldots \ldots \ldots \ldots \ldots \ldots \ldots \ldots \ldots \ldots \ldots \ldots$

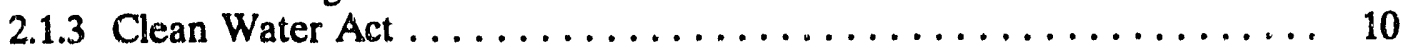

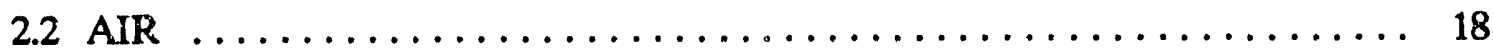

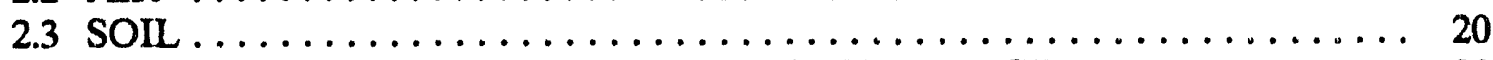

2.4 OTHER "TO-BE-CONSIDERED" (TBC) GUIDANCE . . . . . . . . . 20

3. RADIATION PROTECTION STANDARDS $\ldots \ldots \ldots \ldots \ldots \ldots \ldots \ldots \ldots \ldots$

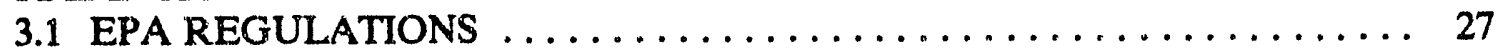

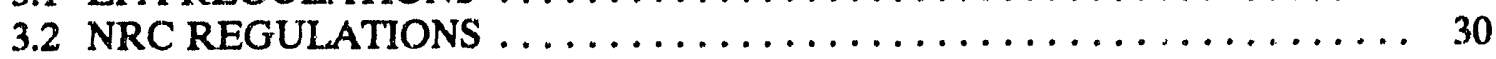

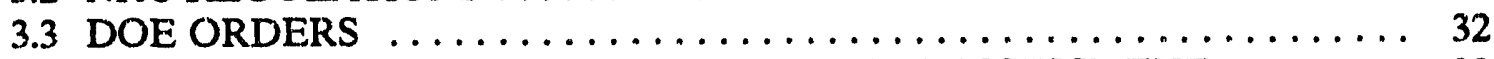

3.4 TBC GUIDANCE FOR RADIOLOGICAL RISK ASSESSMENT . . . . . . 32

4. LOCATION-SPECIFIC ARARS . . . . . . . . . . . . . . . 37

4.1 CAVES, SALT-DOME FORMATIONS, SALT-BED FORMATIONS,

AND UNDERGROUND MINES $\ldots \ldots \ldots \ldots \ldots \ldots \ldots \ldots \ldots \ldots \ldots \ldots$

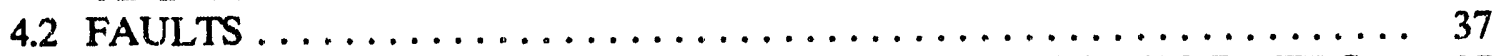

4.3 WILDERNESS AREAS, WILDLIFE REFUGES, AND SCENIC RIVERS .. 37

4.4 WETLANDS AND FLOODPLAINS .................. 44

4.5 HISTORIC SITES AND ARCHEOLOGICAL FINDINGS $\ldots \ldots \ldots \ldots \ldots \ldots 44$

4.6 RARE, THREATENED, OR ENDANGERED SPECIES . . . . . . . . 45

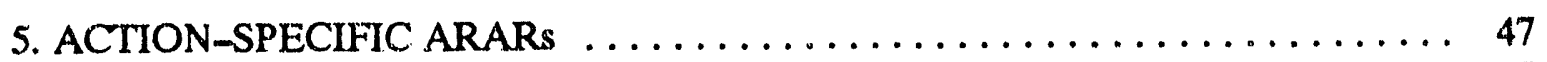

5.1 RESOURCE CONSERVATION AND RECOVERY ACT . . . . . . . . 47

5.1.1 RCRA Subtitle C Regulations ................... 47

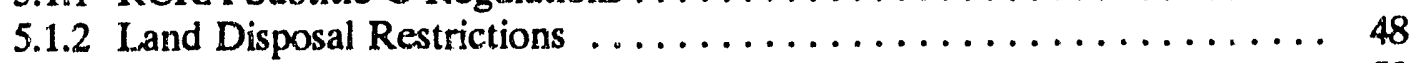

5.1 .3 Corrective Action Requirements ................. 50

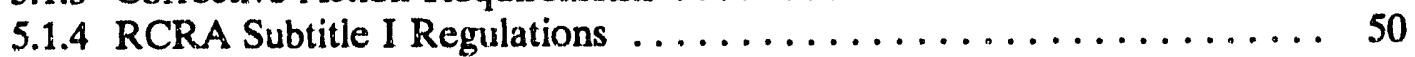

5.1 .5 RCRA Subtitle D Regulations .................... 50

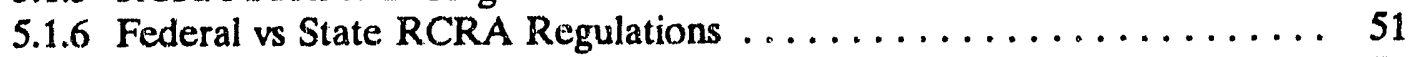

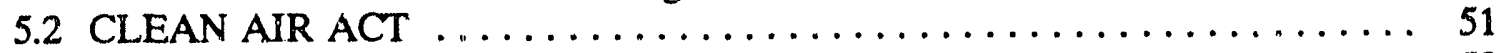

5.3 CLEAN WATER ACT $\ldots \ldots \ldots \ldots \ldots \ldots \ldots \ldots \ldots \ldots \ldots \ldots \ldots \ldots$ 


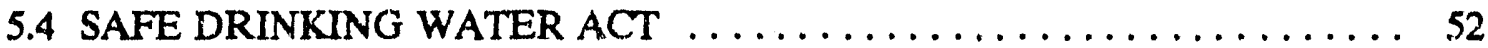

5.5 TOXIC SUBSTANCES CONTROL ACT . . . . . . . . . . . . . 53

5.6 FEDERAL INSECTICIDE, FUNGICIDE, AND RODENTICIDE ACT . . . 53

5.7 OCCUPATIONAL SAFETY AND HEALTH ADMINISTRA'TION $\ldots \ldots \ldots 53$

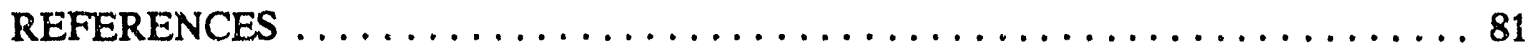




\section{TABLES}

1. Chemical-specific federal ARARs for protection of human health $\ldots \ldots \ldots \ldots$

2. Proposed Sate Drinking Water Act maximum contaminant level goals (MCLGs) and maximum contaminant levels (MCLs) $\ldots \ldots \ldots \ldots \ldots \ldots \ldots \ldots$

3. Federal and Kentucky secondary drinking water regulations $\ldots \ldots \ldots \ldots \ldots \ldots 11$

4. Federal ambient water quality criteria (WQC) for the protection

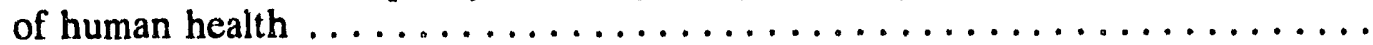

5. Kentucky ambient water quality criteria (WQC) for the protection

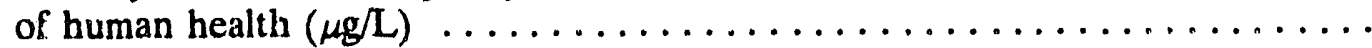

6. Federal ambient water quality criteria for the protection of freshwater organisms $\ldots \ldots \ldots \ldots \ldots \ldots \ldots \ldots \ldots \ldots \ldots \ldots \ldots$

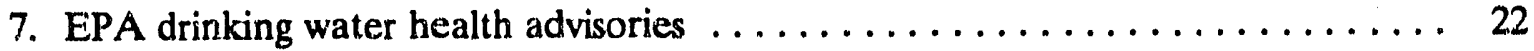

8. Radionuclide-specific ARARs for groundwater and surface water contamination at the Paducah Gaseous Diffusion Plant ................... 28

9. Radiation protection standards that may be ARAR for the Paducah

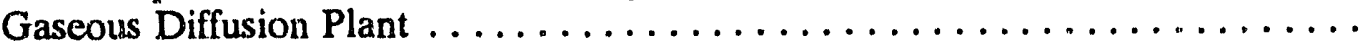

10. TBC guidance from 10 CFR Part 61 - licensing requirements for land disposal of radioactive waste

11. DOE Order 5400.5 "Radiation Protection of the Public and the Environment" . 33

12. DOE Order $5820.2 \mathrm{~A}$ "Radioactive Waste Management" $\ldots \ldots \ldots \ldots \ldots \ldots$

13. Location-specific applicable or relevant and appropriate requirements (ARARs) for the Paducah Gaseous Diffusion Plant . . . . . . . . . . . . . . . .

14. Federally listed and state-listed threatened and endangered species found at West Kentucky Wildlife Management Area and Metropolis Lake . . . . . . . .

15. RCRA Subtitle C - standards for the treatment, storage, and disposal

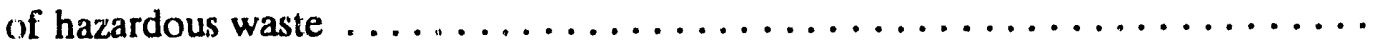

16. Federal RCRA land disposal regulations $\ldots \ldots \ldots \ldots \ldots \ldots \ldots \ldots \ldots$

17. Selected probable action-specific applicable or relevant and appropriate requirements (ARARs) for the Paducah Gaseous Diffusion Plant 


\section{ACRONYMS}

ACL

$\mathrm{ACO}$

AEA

ALARA

ANPRM

ARAR

BDAT

BDT

CAA

CERCLA

CSF

CWA

DCG

DOE

DOI

EO

EPA

FFCA

FIFRA

$F R$

HA

HSWA

ICRP

IRIS

KAR

KAS

KDFW

KNPC

LDR

LLW

$\mathrm{MCL}$

MCLG

NA

NAAQS

NCP

NEPA

NESHAPS

NPDES

NPDWS

NPL

NPRM

NRC

NSDWS
Alternate concentration limit

Administrative Consent Order

Atomic Energy Act

As low as reasonably achievable

Advance Notice of Proposed Rulemaking

Applicable or relevant and appropriate requirement

Best demonstrated available technology

Best demonstrated technology

Clean Air Act

Comprehensive Environmental Response, Compensation, and

Liability Act of 1980

Carcinogen slope factor

Clean Water Act

Derived concentration guide

U. S. Department of Energy

U. S. Department of Interior

Executive Order

U. S. Environmental Protection Agency

Federal Facilities Compliance Agreement

Federal Insecticide, Fungicide, and Rodenticide Act

Federal Register

Health Advisory

Hazardous and Solid Waste Amendments of 1984

International Cornmission on Radiological Protection

Integrated Risk Information System

Kentucky Administrative Regulation

Kentucky Academy of Science

Kentucky Department of Fish and Wildlife

Kentucky Nature Preserves Commission

La id disposal restrictions

Low-level waste

Maximum contaminant level

Maximum contaminant levei goal

Not available

National Ambient Air Quality Standards

National Contingency Plan

National Environmental Policy Act

National Emission Standards for Hazardous Air Pollutants

National Pollutant Discharge Elimination System

National Primary Drinking Water Standards

National Priorities List

Notice of Proposed Rulemaking

Nuclear Regulatory Commission

National Secondary Drinking Water Standards 


$\begin{array}{ll}\text { NSPS } & \text { New Source Performance Standards } \\ \text { ODW } & \text { Office of Drinking Water } \\ \text { OSHA } & \text { Occupational Safety and Health Administration } \\ \text { OSWER } & \text { Office of Solid Waste and Emergency Response } \\ \text { PCB } & \text { Polychlorinated biphenyl } \\ \text { PGDP } & \text { Paducah Gaseous Diffusion Plant } \\ \text { RCRA } & \text { Resource Conservation and Recovery Act } \\ \text { RfC } & \text { Reference Concentration } \\ \text { RfD } & \text { Reference dose } \\ \text { RI/FS } & \text { remedial investigation/feasibility study } \\ \text { SARA } & \text { Superfund Amendments and Reauthorization Act } \\ \text { SDWA } & \text { Safe Drinking Water Act } \\ \text { SEN } & \text { Secretary of Energy Notice } \\ \text { TBC } & \text { To be considered } \\ \text { TRU } & \text { Transuranic } \\ \text { TSCA } & \text { Toxic Substances Control Act } \\ \text { TSD } & \text { Treatment, storage, and disposal facilities } \\ \text { UIC } & \text { Underground injection control } \\ \text { UST } & \text { Underground storage tank } \\ \text { WKWMA } & \text { West Kentucky Wildlife Management Area } \\ \text { WQC } & \text { Water quality criteria }\end{array}$




\section{ACKNOWLEDGMENTS}

The contributions and suggestions of Linda Houlberg, and her knowledge of regulatory changes, have been invaluable in the preparation of this report. The author also wishes to thank Sharon McConathy and Pam Shamblin, Publications Division, for their assistance in technical editing. 


\section{EXECUTTVE SUMMARY}

Section 121 of the Comprehensive Environmental Response, Compensation, and Liability Act (CERCLA) of 1980 specifies that remedial actions for cleanup of hazardous substances found at sites placed on the National Priorities List (NPL) by the U.S. Environmental Protection Agency (EPA) must comply with applicable or relevant and appropriate requirements (ARARs) or standards under federal and state environmental laws. To date, the U.S. Department of Energy (DOE) Paducah Gaseous Diffusion Plant (PGDP) has not been on the NPL. Although DOE and EPA have entered into an Administrative Consent Order (ACO), the prime regulatory authority for cleanup at PGDP will be the Resource Conservation and Recovery Act (RCRA).

This report supplies a preliminary list of available federal and state ARARs that might be considered for remedial response at PGDP in the event that the plant becomes included on the NPL or the ACO is modified to include CERCLA cleanup. A description of the terms "applicable" and "relevant and appropriate" is provided, as well as definitions of chemical-, location-, and action-specific ARARs. ARARs promulgated by the federal government and by the state of Kentucky are listed in tables. In addition, the major provisions of RCRA, the Safe Drinking Water Act, the Clean Water Act, the Clean Air Act, and other acts, as they apply to hazardous and radioactive waste cleanup, are discussed.

In the absence of ARARs, CERCLA $\$ 121$ provides for the use of nonpromulgated federal criteria, guidelines, and advisories in evaluating the human risk associated with remedial action alternatives. Such nonpromulgated standards are classified as "to-beconsidered" guidance. A discussion of available guidance is given, and human-health-effects data are tabulated. Summary tables list the available federal standards and guidance information. 


\section{INTRODUCTION}

The Comprehensive Environmental Response, Compensation, and Liability Act (CERCLA) of 1980 was passed by Congress and signed into law on December 11, 1980 (Public Law 96-510). This act was intended to provide for "liability, compensation, cleanup, and emergency response for hazardous substances released into the environment and the cleanup of inactive waste disposal sites." The Superfund Amendments and Reauthorization Act (SARA), adopted on October 17, 1986 (Public Law 99-499), did not substantially alter the original structure of CERCLA but provided extensive amendments to it.

In particular, $\$ 121$ of CERCLA specifies that remedial actions for cleanup of hazardous substances must comply with requirements or standards under federal or more stringent state environmental laws which are applicable or relevant and appropriate to the hazardous substances or particular circumstances at a site. Inherent in the interpretation of applicable or relevant and appropriate requirements (ARARs) is the assumption that protection of human health and the environment is ensured. The preamble to Subpart E, "Hazardous Substance Response," of the final National Contingency Plan (NCP) (55 FR 8687-8775, March 8, 1990) discusses the identification and use of ARARs as the remedial investigation/feasibility study (RI/FS) progresses for a site. The U.S. Environmental Protection Agency (EPA) has provided two reports entitled CERCLA Compliance with Other Laws Manual, Vols. I and II (EPA 1988, 1989), which are intended as guidance documents for CERCLA compliance with environmental and public health statutes in implementing remedial actions. Much of the information found in this report was developed from those documents. Subpart $\mathbf{K}$ of the NCP, "Federal Facilities," is intended to provide guidance to federal agencies conducting response actions at federal facilities and, when proposed, will be considered for response actions at the Paducah Gaseous Diffusion Plant (PGDP) [Notice of Proposed Rulemaking (NPRM) February 1992, Final Rule expected November 1992].

The U.S. Department of Energy (DOE) owns PGDP, which is managed by Martin Marietta Energy Systems, Inc. PGDP is an operating uranium enrichment facility and has functioned as such since 1952. PGDP is in the Ohio River Basin, situated -3.7 miles south of the Ohio River; Big Bayou Creek and Little Bayou Creek are located west and east of the plant, respectively, and receive effluents and runoff from the plant. These creeks join downgradient from the plant before flowing into the Ohio River. The plant is located in a predominantly rural area, surrounded by scattered homes and farms. Three groundwater systems have been identified in the area. Although the regional gravel aquifer is the primary source of drinking water for area wells, the shallow groundwater system supplies drinking water to several residences east of the plant. The deep groundwater system is not typically utilized for drinking water in this area. PGDP operates a "nontransient, noncommunity" public water treatment facility.

In August, 1988, off-site sampling of private drinking water wells in the vicinity of PGDP indicated the presence of various contaminants associated with the uranium enrichment process, including trichloroethylene (TCE), TCE degradation products, and ${ }^{99} \mathrm{Tc}$. On November 23, 1988, EPA and DOE entered into an Administrative Consent Order (ACO) under $\$ 104$ and 106 of CERCLA. The objectives of the ACO were to determine the extent 
of the threat to human health and the environment from off-site contamination of groundwater from PGDP and to establisit workplans and schedules for developing and implementing response actions according to CERCLA. The objectives of the ACO only include the off-site groundwater contaminati on and the on-site sources of that contamination. This investigation is being conducted in two phases. The purpose of Phase I is to determine the nature and extent of off-site groundwater contamination, while Phase II will evaluate the on-site sources of the groundwater contamination.

A final Hazardous and Solid Waste Amendments (HSWA) permit for PGDP was issued by EPA on July 16, 1991 (effective August 19, 1991) (EPA Permit Number KY8 890008 982). Appendix A-1(a) of this permit contains a list of all of the known solid waste management units (SWMUs) and areas of concern requiring a Resource Conservation and Recovery Act (RCRA) Facility Investigation (RFI), and Appendix A-1(b) contains a list of all of the known SWMUs and areas of concern that are suspected of contributing to off-site releases. These latter sites are the focus of the Phase II study under the ACO. However, in the EPA Region IV response to the DOE/Energy Systems comments regarding the draft EPA HSWA permit for PGDP, it is stated that although the investigations and assessments stipulated in the ACO are to be implemented under CERCLA, the prime regulatory authority for cleanup will be RCRA and the PGDP HSWA permit (Response No. 1 and Figure 1 of the Response). According to EPA, this concept would prevail unless the ACO is modified to include site cleanup or unless PGDP is placed on the National Priorities List (NPL).

The purpose of this report is to supply a preliminary list of available federal and state chemical-, location-, and action-specific ARARs that might be considered for PGDP in the event that the ACO is modified or that the site is placed on the NPL and remediation proceeds under CERCLA. The process of ARAR identification is an iterative one that is continually changing as the RI/FS progresses; actual ARARs are identified only on a sitespecific basis. Therefore, this list of ARARs represents a compilation of potential ARARs, of which subsets will be used or additional ARARs added as site-specific contamination at PGDP is characterized and remedial actions selected. No attempt will be made in this report to determine whether the regulations will be either applicable or relevant and appropriate. This will be done on a site-specific basis.

It is understood that DOE will comply with the requirements of the National Environmental Policy Act (NEPA) as specified in DOE Order 5440.1D (National Environmental Policy Act Compliance Program). Further, DOE Order 5400.4 (Comprehensive Environmental Response, Compensation, and Liability Act Requirements) calls for integration of NEPA and CERCLA requirements for DOE remedial actions at CERCLA sites. This issue has been reaffirmed in the Secretary of Energy Notice of February 5, 199J (SEN-15-90), which was issued to ensure that DOE's NEPA activities are carried out in a centralized and uniform manner. Therefore, the regulations found in NEPA will not be addressed in this report as ARARs.

Similarly, DOE addresses occupational safety in DOE Orders 5480.11 (Radiation Protection for Occupational Workers), 5480.4 (Environmental Protection, Safery, and Health Protection Standards), 5483.1A (Occupational Safety and Health Program for Contractors at GOCO Facilities), and 5480.9 (Constriction Safety and Health Program). However, ARARs apply to those federal and state regulations that are designed to protect the environment, and do not generally apply to occupational safety regulations. Therefore, the DOE orders related to occupational safety and the regulations promulgated by the Occupational Safety and Health 
Administration (OSHA) are not addressed as ARARs unless they specifically apply to remedial action goals.

The following is a listing of the definitions of terms used throughout this report:

Applicable requirements are "those cleanup standards, standards of control, and other substantive environmental protection requirements, criteria, or limitations promulgated under federal or state law that specifically address a hazardous substance, pollutant, contaminant, remedial action, location, or other circumstance at a CERCLA site" (52 FR 32496, August 27, 1987).

Relevant and appropriate requirements are "those cleanup standards, standards of control, and other substantive environmental protection requirements, criteria, or limitations promulgated under federal or state law that, while not applicable to a hazardous substance, pollutant, contaminant, remedial action, location, or other circumstance at a CERCLA site, address problems or situations sufficiently similar to those encountered at the CERCLA site that their use is well suited to the particular site" (52 FR 32496).

Requirements under federal or state law may be either applicable or relevant and appropriate to CERCLA cleanup actions, but not both. However, requirements must be both relevant and appropriate for compliance to be necessary. In the case where a federal and a state ARAR are available, or where there are two potential ARARs addressing the same issue, the more stringent regulation must be selected. However, CERCLA \$121(d)(4) provides several ARAR waiver options that may be invoked, providing that the basic premise of protection of human health and the environment is not ignored. A waiver is available for state standards that have not been applied uniformly in similar circumstances across the state. In addition, CERCLA $\$ 121(\mathrm{~d})(2)(\mathrm{C})$ forbids state standards that effectively prohibit land disposal of hazardous substances.

CERCLA on-site remedial response actions must only comply with the substantive requirements of a regulation and not the administrative requirements to obtain federal, state, or local permits [CERCLA \$121(e)]. To ensure that CERCLA response actions procsed as rapidly as possible, EPA has reaffirmed this position in the final NCP (55 FR 8756). Substantive requirements pertain directly to the actions or conditions at a site, while administrative requirements facilitate their implementation. EPA recognizes that certain of the administrative requirements such as consultation with state agencies, reporting, etc., are accomplished through the state involvement and public participation requirements of the NCP. These administrative requirements should be observed if they are useful in determining cleanup standards at the site (55 FR 8757).

In the absence of federal- or state-promulgated regulations, there are many criteria, advisories, guidance values, and proposed standards that are not legally binding but may serve as useful guidance for setting protective cleanup levels. These are not potential ARARs but are "to-be-considered" (TBC) guidance. 


\section{CHEMICAI-SPECIFIC ARARs}

"Chemical-specific requirements set health or risk-based concentration limits or discharge limitations in various environmental media for specific hazardous substances, pollutants, or contaminants" ( 52 FR 32496). These requirements generally set protective cleanup levels for the chemicals of concern in the designated media or else indicate a safe level of discharge that may be incorporated when considering a specific remedial activity. A variety of hazardous substances, including radioactive, nonradioactive, and mixed hazardous wastes have been disposed of for many years at PGDP. Metals, organic chemicals, and radionuclides have been deterted in all environmental media: air, surface water, groundwater, soil, and sediment.

Although limited in number, chemical-specific standards have been established under several statutes, including RCRA, the Safe Drinking Water Act (SDWA), the Clean Water Act (CWA), and the Clean Air Act (CAA).

\section{GROUNDWATER AND SUR FACE WATER}

Table 1 lists available chemical-specific ARARs that have been promulgated under federal law for contaminated groundwater or surface water that may be used for drinking water at PGDP. The regulations for air are described in Sects. 2.2 and 5.2.

As stated in the NCP (55 FR 8666), the goal of EPA's approach to cleanup of contaminated groundwater is to return usable groundwater to its beneficial use within a given time frame that is reasonable for the particular circumstances at a CERCLA site. Classes I and IIA represent current sources of drinking water of varying value; Class IIB represents potential sources of drinking water. Groundwater at PGDP has been used for private drinking water and therefore may be classified as Class I or Class II groundwater.

In the NCP, EPA states the preference for SDWA maximum contaminant levels (MCLs) and nonzero maximum contaminant level goals (MCLGs) or other health-based standards, criteria, or guidance for cleanup of Class I and Class II groundwater at CERCLA sites (55 FR 8732). Alternate concentration limits (ACLs) may also be used when active restoration of the groundwater to MCLs or nonzero MCLGs is not practicable (55 FR 8754).

\subsubsection{Resource Conservation and Recovery Act}

Subtitle C of RCRA lists maximum concentration levels for 14 chemicals; the concentration of these chemicals in groundwater at the plant boundary of a RCRA-permitted treatment, storage, or disposal (TSD) facility may not exceed the stated maximum. concentration level [Title 40, Code of Federal Regulations, Part 264, 694 (40 CFR 264.94)]. In addition, background concentrations or ACLs are established in 40 CFR 264.94 as groundwater protection standards. Table 1 lists RCRA MCLs; however, as mentioned above, EPA has specified SDWA MCLs for cleanup of Class I and Class II groundwater. This approach is consistent with the substantive requirements of RCRA MCLs, ACLs, or background limits (53 FR 51433). The newly promulgated regulations for municipal landfills (56 FR 50978, October 9, 1991) establish all SDWA MCLs by reference, ACLs, or background concentrations as groundwater protection standards (40 CFR 258.55). Although not legally applicable to the remedial action sites found at PGDP, these may prove relevant 


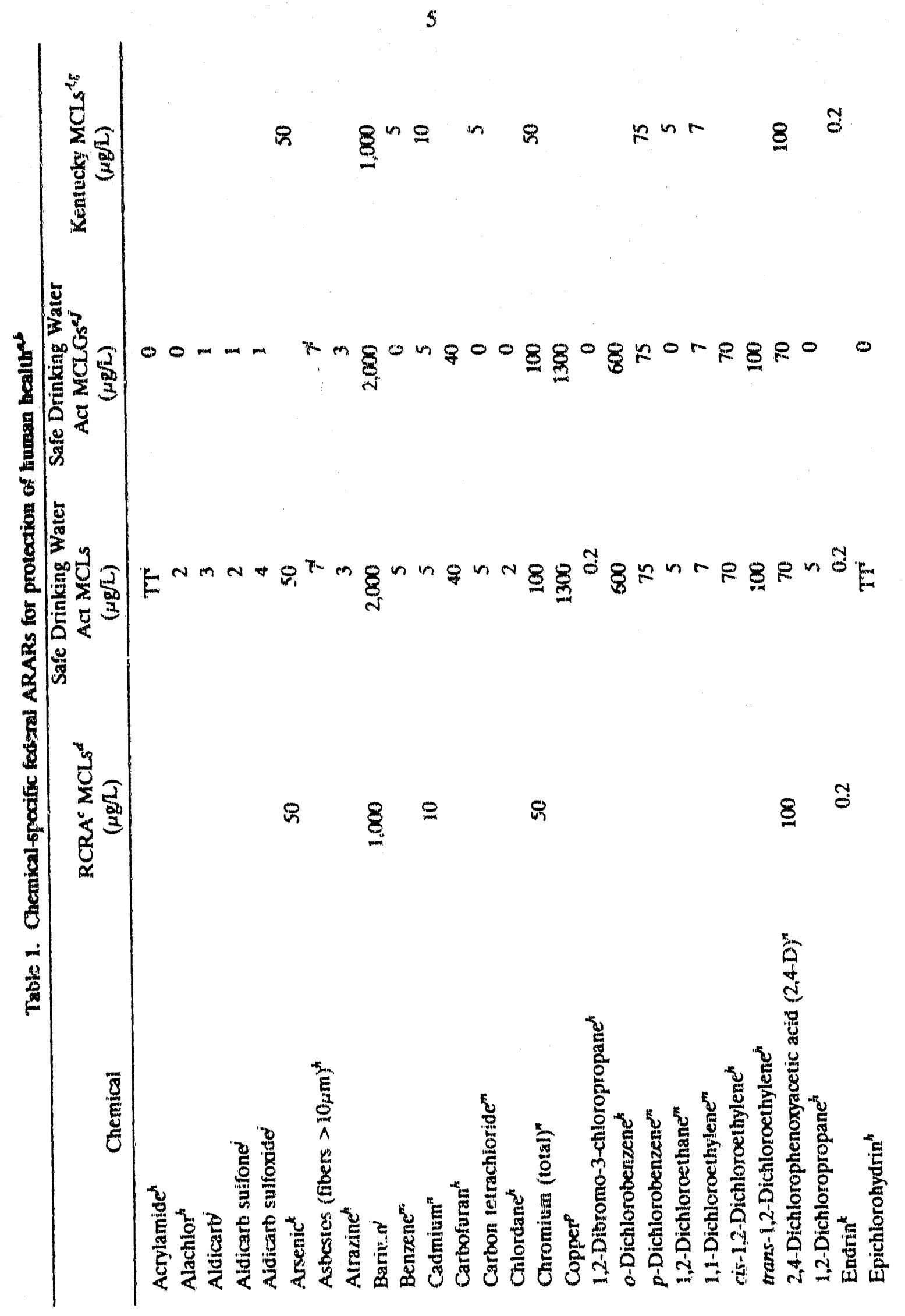




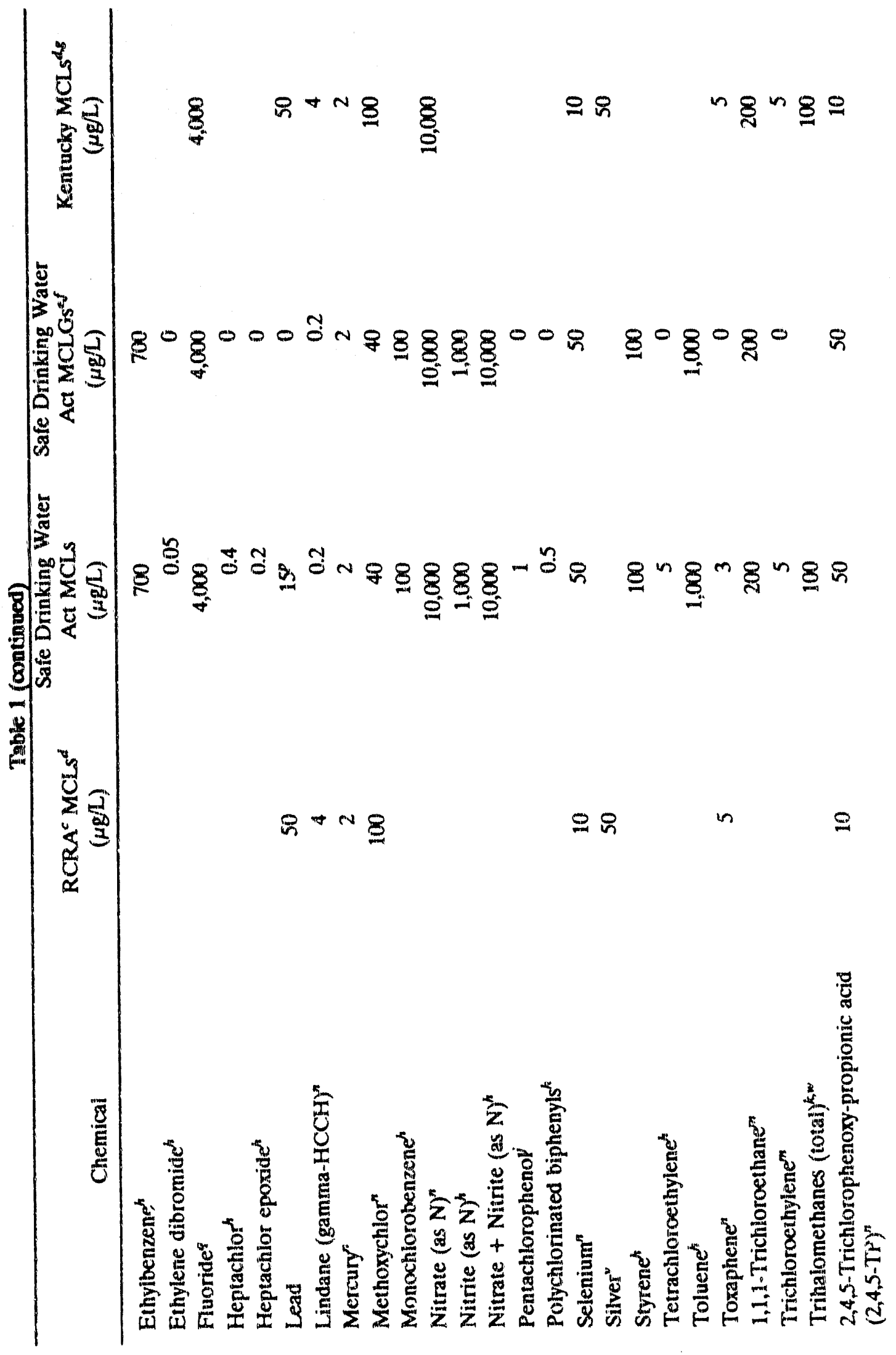




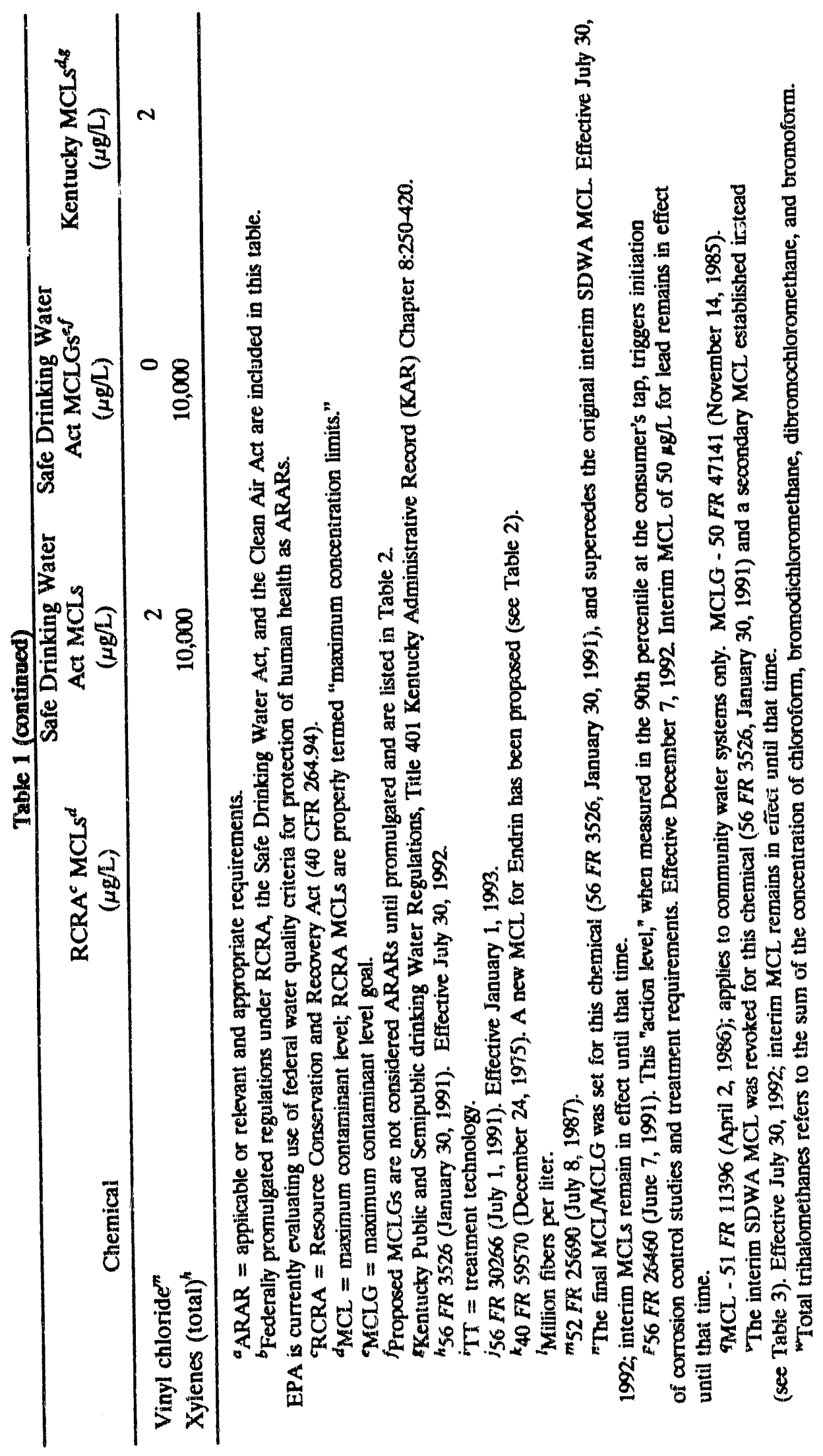


and appropriate for cleanup of groundwater at some of the sites. ACLs may be calculated for chemicals without an MCL using a risk-based approach (56 FR 51026).

\subsection{Safe Drinking Water Act}

EPA has promulgated primary and secondary drinking water regulations applicable to public water systems that have at least 15 service connections or serve an average of at least 25 people daily at least 60 days of the year. National Primary Drinking Water Standards (NPDWS) are established in 40 CFR 141 and include MCLs and MCLGs. New drinking water standards promulgated for eight synthetic organic chemicals (52 FR 25690, July 8, 1987) added a new category of suppliers referred to as noncommunity, nontransient systems that regularly serve at least 25 people for 6 months of the year. Table 1 lists SDWA MCLs and MCLGs.

MCLs are enforceable standards that take into consideration human-health effects, available treatment technologies, and costs of treatment. MCLGs are strictly health-based standards that disregard cost or treatment feasibility and are not legally enforceable. MCLs are legally applicable to water "at the tap" but ase not applicable to cleanup of groundwater or surface water. However, they may be considered as relevant and appropriate in situations where groundwater or surface water may be used for drinking water. CERCLA \$121(d)(2)(A) specifically mentions that remedial actions must require a level or standard of control that at least attains MCLGs and federal ambient water quality criteria (WQC) where such goals or criteria are relevant and appropriate under the circumstances of the release. Although MCLGs and WQC are nonenforceable guidelines, Congress elevated them to a higher status by specifically mentioning them in CERCLA. Therefore, promulgated MCLGs are listed in Table 1. At present, EPA is planning to use the SDWA MCLs for remedial action compliance for carcinogens which have an MCLG of zero and any nonzero MCLG for systemic toxicants (55 FR 8752).

The SDWA amendments of 1986 mandated that EPA propose MCLs and MCLGs for contaminants in drinking water which may cause any adverse effect on human health. Proposed MCLs/MCLGs have appeared in 55 FR 30370 (July 25, 1990). Table 2 lists the proposed MCLs/MCLGs. When the proposed MCLs/MCLGs are promulgated and become effective, they will be considered relevant and appropriate for cleanup at PGDP (a final rule is expected February 1992).

EPA has revised its drinking water standards for lead and copper, eliminating the MCL and replacing it with an "action level" of $15 \mu \mathrm{g} / \mathrm{L}$ and $1300 \mu \mathrm{g} / \mathrm{L}$, respectively (56 FR 26460, June 7, 1991), applicable to community and nontransient, noncommunity water systems. Included in the national primary drinking water regulation is a treatment technique requirement. If the "action level" for lead or copper is exceeded at the tap, a state is required to analyze source-water samples and to decide what treatment levels are necessary to minimize lead or copper levels delivered to users from the affected distribution system. Because PGDP operates a nontransient, noncornmunity public water system, these "action levels" will be legally applicable at PGDP. In the event that the amount of lead or copper at the tap exceeds the "action levels" (see the final rule for a description of the monitoring techniques), the source-water treatment requirements may become ARARs for cleanup of lead-or copper-contaminated groundwater at PGDP. 
Table 2. Proposed Safe Drinking Water Act maximum contaminant level goals (MCLGs) and maximum contaminant levels (MCLs)"

\begin{tabular}{|c|c|c|}
\hline Chemical & $\begin{array}{c}\text { Proposed MCLG } \\
(\mu \mathrm{g} / \mathrm{L})\end{array}$ & $\begin{array}{c}\text { Proposed MCL } \\
(\mu \mathrm{g} / \mathrm{L})\end{array}$ \\
\hline Adipates [di(ethylhexyl)adipate] & 500 & 500 \\
\hline Antimony & 3 & $10 / 5^{b}$ \\
\hline Beryllium & 0 & 1 \\
\hline Cyanide & 200 & 200 \\
\hline Dalapon & 200 & 200 \\
\hline Dichloromethane (methylene chloride) & 0 & 5 \\
\hline Dinoseb & 7 & 7 \\
\hline Diquat & 20 & 20 \\
\hline Endothall & 100 & 100 \\
\hline Endrin & 2 & 2 \\
\hline Glycophosate & 700 & 700 \\
\hline Hexachlorobenzene & 0 & 1 \\
\hline Hexachlorocyclopentadiene & 50 & 50 \\
\hline Nickel & 100 & 100 \\
\hline Oxamyl (Vydate) & 200 & 200 \\
\hline \multicolumn{3}{|l|}{ Polyaromatic hydrocarbons (PAHs) } \\
\hline Benzo(a)pyrene & 0 & 0.2 \\
\hline Benz(a)anthracene $e^{c}$ & 0 & 0.1 \\
\hline Benzo(b)fluoranthene ${ }^{c}$ & 0 & 0.2 \\
\hline Benzo(k)fluoranthene ${ }^{c}$ & 0 & 0.2 \\
\hline Chrysene ${ }^{c}$ & 0 & 0.2 \\
\hline Dibenz(a,h)anthracene $e^{c}$ & 0 & 0.3 \\
\hline Indenopyrene $e^{c}$ & 0 & 0.4 \\
\hline Phthalates [di(ethylhexyl) phthalate] & 0 & 4 \\
\hline Picloram & 500 & 500 \\
\hline Simazine & 1 & 1 \\
\hline Sulfate $^{d}$ & $400,000 / 500,000$ & $400,000 / 500,000$ \\
\hline Thallium & 0.5 & $2 / 1^{d}$ \\
\hline $2,3,7,8$-Tetrachlorodibenzo- $p$-dioxin (dioxin) & 0 & 0.00005 \\
\hline 1,2,4-Trichlorobenzene & 9 & 9 \\
\hline 1,1,2-Trichloroethane & 3 & 5 \\
\hline
\end{tabular}

a55 FR 30370 (July 25, 1990). A final rule is expected March 1992.

${ }^{b}$ EP. A proposes two MCLs for public comment based on two practical quantitation limits.

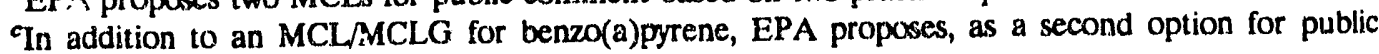
comment, MCIS/MCLGs for these PAHs.

${ }^{d}$ Two options are presented for public comment.

The Kentucky Administrative Regulation (KAR) lists MCLs for public water systems [Kentucky Public and Semipublic Drinking Water Regulations, Title 401 KAR 8:250 (inorganic chemicals); 8:400 (organic chemicals); 8:420 (volatile organics); 8:500 (trihalomethanes); and 8:550 (radionuclides)]. In most cases, the Kentucky MCLs are identical to the federal MCLs; however, several of the recently adopted federal MCLs are less stringent than the state MCLs, and therefore the more stringent Kentucky MCLs would be 
relevant and appropriate for cleanup of groundwater and surface water that may be used for drinking water purposes at PGDP. However, the Kentucky Office of Drinking Water plans to revise the state drinking water regulations to adopt the federal MCLs when they become effective (July 30,1992). The revised state MCLs would be expected to become effective mid1993 (Schiefferle 1991).

National Secondary Drinking Water Standards (NSDWS) regulate contaminants that affect the aesthetic qualities related to public acceptance of drinking water and are implemented in 40 CFR 143.3 as secondary maximum contaminant levels (SMCLs). These regulations are not federally enforceable, but rather are intended to serve as guidelines for use by states in regulating water supplies. Kentucky has promulgated secondary drinking water regulations in Title 401 KAR 8:600 (see Table 3). The regulations stipulate that suppliers of public and semipublic drinking water systems treat existing sources of water so that the water meets the secondary standards listed in Table 3 . In that context, the secondary standards would not be legally applicable to cleanup of groundwater or surface water, but may be considered as relevant and appropriate in instances where these media may provide private drinking water sources. EPA has proposed NSDWS for ten additional contaminants (54 FR 22062, May 22, 1989; 55 FR 30370 July 25, 1990). When these are promulgated they will be incorporated into the Kentucky secondary drinking water regulations (Schiefferle 1991); therefore, they are included in Table 3 for completeness. A final rule for two of the proposed NSDWS has appeared (56 FR 3526, January 30, 1991).

\subsubsection{Clean Water Act}

CERCLA \$121(d)(2)(A) specifically states that remedial actions shall at least attain federal ambient WQC established under the CWA if they are relevant and appropriate. In determining whether any WQC are relevant and appropriate, one must consider the "designated or potential use of the surface or groundwater, the environmental media affected, the purposes for which the criteria were developed, and the latest information available" [CERCLA $\$ 121(\mathrm{~d})(2)(B)]$. Federal WQC are derived for the protection of freshwater aquatic organisms and for the protection of human health from the consumption of contaminated drinking water and/or aquatic organisms.

Table 4 lists ambient WQC for the protection of human health. EPA has derived WQC for ingestion of drinking water and aquatic organisms and for the ingestion of aquatic organisms alone. Since neither of these categories is relevant and appropriate for consideration of contaminated groundwater, WQC derived for the ingestion of drinking water alone are also included in Table 4. The EPA Region IV Water Quality Standards Unit has adjusted WQC for human health based on recent human toxicity information [Reference Doses (RfDs) and carcinogen slope factors (CSFs)] obtained from the EPA Integrated Risk information System (IRIS). The values presented in Table 4 reflect the EPA Region IV revised WQC and are current as of October 1991 (EPA 1991a). They are intended to supercede all previous WQC.

As part of the federal requirement for a triennial review of state water quality standards, Kentucky will consider adopting the EPA Region IV WQC (late 1993); however, to date, Kentucky has not adopted the revised EPA Region IV WQC into their promulgated regulations (Anderson 1991). Therefore, the current Kentucky WQC are listed in Table 5. Because the state WQC are promulgated regulations, they will be legally applicable to cleanup of surface water that may be used for domestic water supplies. 
Table 3. Federal and Kentucky secondary drinking water regulations

\begin{tabular}{|c|c|c|}
\hline Chemical & $\begin{array}{c}\text { Federal SMCLa } \\
(\mathrm{mg} / \mathrm{L})\end{array}$ & $\begin{array}{c}\text { Kentucky SMCL } \\
(\mathrm{mg} / \mathrm{L})\end{array}$ \\
\hline Aluminum & $0.05-0.02^{\text {csd }}$ & \\
\hline Chloride & 250 & 250 \\
\hline Color & 15 (color units) & 15 (color units) \\
\hline Copper & 1 & 1 \\
\hline o-Dichlorobenzene & $0.01^{e}$ & \\
\hline$p$-Dichlorobenzene & $0.005^{e}$ & \\
\hline Ethylbenzene & $0.03^{e}$ & \\
\hline Fluoride & 2.0 & 2.0 \\
\hline Hexachlorocyclopentadiene & $0.008 f$ & \\
\hline Fydrogen sulfide & & 0.05 \\
\hline Iron & 0.3 & 0.3 \\
\hline Manganese & 0.05 & 0.05 \\
\hline Odor & $\begin{array}{l}3 \text { (threshold odor } \\
\text { number) }\end{array}$ & $\begin{array}{l}3 \text { (threshold odor } \\
\text { number) }\end{array}$ \\
\hline Pentachlorophenol & $0.03^{e}$ & \\
\hline Phenols & & 0.001 \\
\hline $\mathrm{pH}$ & $6.5-8.5$ & $6.5-8.5$ \\
\hline Silver & $0.1^{c}$ & \\
\hline Styrene & $0.01^{e}$ & \\
\hline Sulfate & 250 & 250 \\
\hline Toluene & $0.04^{e}$ & \\
\hline TDS (total dissolved solids) & 500 & 500 \\
\hline Xylene & $0.02^{e}$ & \\
\hline Zinc & 5 & 5 \\
\hline
\end{tabular}

${ }^{a} \mathrm{SMCL}=$ secondary maximum contaminant level. Final rule [44 FR 42198 (Juky 19, 1979)].

bKentucky Public and Semipublic Drinking Water Regulations, Title 401, KAR 8:600

${ }^{c}$ NSDWS final rule [56 FR 3526 (January 30, 1991)]. Effective date July 30, 1992.

${ }^{d}$ Level recommended to prevent posttreatment precipitation in the distribution system.

'Proposed NSDWS (54 FR 22062, May 22, 1989). EPA has deferred setting final standards for these chemicals pending further study (56 FR 3572, January 30, 1991).

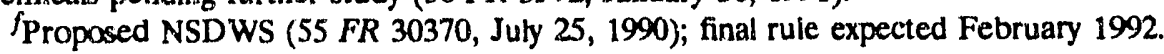


Table 4. Federal ambient water quality criteria (WQC) for the protection of human health $(\mu \mathrm{g} / \mathrm{L})$

\begin{tabular}{|c|c|c|c|}
\hline Chemical & $\begin{array}{l}\text { WQC for aquatic } \\
\text { organisms and } \\
\text { drinking water }\end{array}$ & $\begin{array}{l}\text { WQC for aquatic } \\
\text { organisms alone }^{a}\end{array}$ & $\begin{array}{c}\text { WQC for } \\
\text { drinking water } \\
\text { alone }\end{array}$ \\
\hline Acenaphthene & $20(\text { org) })^{c}$ & NA & 2,100 \\
\hline Acrolein & 320 & 780 & \\
\hline Acrylonitrile & $0(0.059)$ & $0(0.67)$ & $0(0.065)$ \\
\hline Aldrin & $0(1.3 \mathrm{E}-04)^{d}$ & $0(1.4 \mathrm{E}-04)$ & $0(2.1 \mathrm{E}-03)$ \\
\hline Anthracene & $9,567.2$ & 107,692 & 10,500 \\
\hline Antimony & 14 & 4,308 & 14 \\
\hline Arsenic & $0(0.018)$ & $0(0.14)$ & $0(0.02)$ \\
\hline Asbestos & $\begin{array}{c}0(30,000 \\
\text { fibers } / \mathrm{L})\end{array}$ & NA & \\
\hline Benzene & $0(1.18)$ & $0(71.28)$ & $0(1.2)$ \\
\hline Benzidine & $0(1.2 \mathrm{E}-04)$ & $0(5.4 E-04)$ & $0(1.5 \mathrm{E}-04)$ \\
\hline Benzo(a)anthracene & $0(2.8 \mathrm{E}-03)$ & $0(0.031)$ & \\
\hline Benzo(a)pyrene & $0(2.8 \mathrm{E}-03)$ & $0(0.031)$ & \\
\hline Benzo(k)fluoranthene & $0(2.8 \mathrm{E}-03)$ & $0(0.031)$ & \\
\hline 3,4-Benzofluoranthene & $0(2.8 E-03)$ & $0(0.031)$ & \\
\hline Beryllium & $0(7.7 \mathrm{E}-03)$ & $0(0.13)$ & $0(8.1 E-03)$ \\
\hline Bromoform & $0(4.38)$ & $0(363.5)$ & $0(4.4)$ \\
\hline Carbon tetrachloride & $0(0.254)$ & $0(4.42)$ & $0(0.27)$ \\
\hline Chlordane & $0(5.8 \mathrm{E}-04)$ & $0(5.9 \mathrm{E}-04)$ & $0(0.03)$ \\
\hline \multicolumn{4}{|l|}{ Chlorinated benzenes } \\
\hline Monochlorobenzene & 20 (org) & NA & 700 \\
\hline 1,2-Dichlorobenzene & 2,667 & 17,432 & 3,150 \\
\hline 1,3-Dichlorobenzene & 400 & 2,600 & \\
\hline 1,4-Dichlorobenzene & 400 & 2,600 & \\
\hline 1,2,4,5-Tetrachlorobenzene & 38 & 48 & \\
\hline Pentachlorobenzere & 74 & 85 & \\
\hline Hexachlorobenzene & $0(7.5 \mathrm{E}-04)$ & $0(7.7 E-04)$ & \\
\hline \multicolumn{4}{|l|}{ Chlorinated ethanes } \\
\hline 1,2-Dichloroethane & $0(0.38)$ & $0(98.6)$ & $0(0.38)$ \\
\hline 1,1,1-Trichloroethane & 3,094 & 173,077 & 3,150 \\
\hline 1,1,2-Trichloroethane & $0(0.605)$ & $0(41.99)$ & $0(0.61)$ \\
\hline 1,1,2,2-Tetrachloroethane & $0(0.172)$ & $0(10.8)$ & $0(0.175)$ \\
\hline Hexachloroethane & $0(1.95)$ & $0(8.85)$ & $0(2.5)$ \\
\hline \multicolumn{4}{|l|}{ Chlorinated phenols } \\
\hline 2-Chlorophenol & 0.1 (org) ${ }^{c}$ & NA & 175 \\
\hline 2,4-Dichlorophenol & 0.3 (org) $^{c}$ & NA. & 105 \\
\hline 2,4,5-Trichlorophenol & $1.0(\mathrm{org})^{c}$ & NA & \\
\hline 2,4,6-Trichlorophenol & $0(2.1)$ & $0(6.5)$ & $0(3.2)$ \\
\hline Pentachlorophenol & $0(0.28)$ & $0(8.2)$ & $0(0.29)$ \\
\hline 3-Methyl-4-chlorophenol & 3,000 (org) ${ }^{c}$ & NA & \\
\hline \multicolumn{4}{|l|}{$\begin{array}{l}\text { 3-Mernyl-4-cnioropnenot } \\
\text { Chloroalkyl ethers }\end{array}$} \\
\hline bis-(Chloromethyl) ether & $0(1.6 \mathrm{E}-04)$ & $0(0.077)$ & $0(1.6 \mathrm{E}-04)$ \\
\hline bis-(2-Chloroethyl) ether & $0(0.031)$ & $0(1.42)$ & $0(0.032)$ \\
\hline bis-(2-Chloroisopropyl) ether & 1,389 & 174,400 & 1,400 \\
\hline Chlorodibromomethane & $0(0.412)$ & $0(34.2)$ & $0(0.417)$ \\
\hline Chloroform & $0(5.67)$ & $0(470.8)$ & $0(5.74)$ \\
\hline
\end{tabular}


13

Table 4. (continued)

\begin{tabular}{|c|c|c|c|}
\hline Chemical & $\begin{array}{l}\text { WQC for aquatic } \\
\text { organisms and } \\
\text { drinking water }\end{array}$ & $\begin{array}{l}\text { WQC for aquatic } \\
\text { organisms alone }^{a}\end{array}$ & $\begin{array}{c}\text { WQC for } \\
\text { drinking water } \\
\text { alone }^{b}\end{array}$ \\
\hline Chromium (III) & 33,300 & 673,077 & 35,000 \\
\hline Chrysene & $0(2.8 \mathrm{E}-03)$ & $0(0.031)$ & \\
\hline Copper & 1,000 (org) & NÀ & \\
\hline DDD & $0(8.3 \mathrm{E}-04)$ & $0(8.3 \mathrm{E}-04)$ & $0(0.15)$ \\
\hline DDE & $0(5.9 \mathrm{E}-04)$ & $0(5.9 \mathrm{E}-04)$ & $0(0.103)$ \\
\hline DDT & $0(5.9 \mathrm{E}-04)$ & $0(5.9 \mathrm{E}-04)$ & $0(0.103)$ \\
\hline $\operatorname{Dibenz}(\mathrm{a}, \mathrm{h})$ anthracene & $0(2.8 \mathrm{E}-03)$ & $0(0.031)$ & \\
\hline 3,3'-Dichlorobenzidine & $0(0.04)$ & $0(0.77)$ & $0(0.078)$ \\
\hline Dichlorobromomethane & $0(0.27)$ & $0(22.1)$ & $0(0.27)$ \\
\hline Dichlorodifluoromethane & $0(5.67)$ & $0(470.8)$ & \\
\hline 1,1-Dichloroethylene & $0(0.06)$ & $0(3.2)$ & $0(0.058)$ \\
\hline \multicolumn{4}{|l|}{$\begin{array}{l}\text { Dichloropropanes/Dichloro- } \\
\text { propenes }\end{array}$} \\
\hline cis-1,3-Dichloropropene & 10.4 & 1,691 & 10.5 \\
\hline trans-1,3-Dichloropropene & 10.4 & 1,691 & 10.5 \\
\hline Dieldrin & $0(1.4 \mathrm{E}-04)$ & $0(1.4 \mathrm{E}-04)$ & $0(2.2 \mathrm{E}-03)$ \\
\hline 2,4-Dimethylphenol & 400 (org) ${ }^{c}$ & $\mathrm{NA}$ & 200 \\
\hline 2,4-Dinitrotoluene & $0(0.11)$ & $0(9.1)$ & \\
\hline 1,2-Diphenylhydrazine & $0(0.041)$ & $0(0.54)$ & $0(0.044)$ \\
\hline Endosulfan $(a-, b-)$ & 0.932 & 1.99 & 1.8 \\
\hline Ethylbenzene & 3,120 & 28,718 & 3,500 \\
\hline Fluoranthene & 295.5 & 374.6 & 1,400 \\
\hline Fluorene & $1,275.6$ & $14,358.5$ & 1,400 \\
\hline Halomethanes & $0(5.67)$ & $0(470.8)$ & \\
\hline Heptachlor & $0(2.1 E-04)$ & $0(2.1 E-04)$ & $0(8.1 \mathrm{E}-03)$ \\
\hline Heptachlor epoxide & $0(1.0 \mathrm{E}-04)$ & $0(1.0 \mathrm{E}-04)$ & $0(3.8 \mathrm{E}-03)$ \\
\hline Hexachlorobutadiene & $0(0.45)$ & $0(49.7)$ & $0(0.45)$ \\
\hline \multicolumn{4}{|l|}{$\begin{array}{l}\text { Hexacniorooutadene } \\
\text { Hexachlorocyclohexanes (HCCH) }\end{array}$} \\
\hline Alpha-HCCH & $0(0.004)$ & $0(0.013)$ & $0(5.5 E-03)$ \\
\hline Beta-HCCH & $0(0.014)$ & $0(0.046)$ & $0(0.02)$ \\
\hline Gamma-HCCH & $0(0.019)$ & $0(0.063)$ & \\
\hline Hexachlorocyclopentadiene & 1 (org) ${ }^{c}$ & $\mathbf{N A}$ & 245 \\
\hline Indeno $(1,2,3-c d)$ pyrene & $0(2.8 \mathrm{E}-03)$ & $0(0.031)$ & \\
\hline Isophorone & $0(8.42)$ & $0(599.7)$ & $0(8.5)$ \\
\hline Mercury & 0.151 & 0.153 & \\
\hline Methyl bromide & 48 & 4,020 & 49 \\
\hline Methyl chloride & $0(5.67)$ & $0(470.8)$ & \\
\hline Methylene chloride & $0(4.65)$ & $0(1,578)$ & $0(4.7)$ \\
\hline Nickel & 607 & 4,584 & 700 \\
\hline Nitrobenzene & 17.3 & 1,863 & 17.5 \\
\hline \multicolumn{4}{|l|}{ Nitrophenols } \\
\hline 2,4-Dinitrophenol & 69.7 & 14,264 & 70 \\
\hline 2,Methyl-4,6-dinitrophenol & 13.4 & 765 & \\
\hline \multicolumn{4}{|l|}{ Nitrosamines } \\
\hline n-Nitrosodimethylamine & $0(6.9 E-04)$ & $0(8.12)$ & $0(6.8 \mathrm{E}-04)$ \\
\hline n-Nitrosodiphenylamine & $0(4.95)$ & $0(16.2)$ & $0(7.1)$ \\
\hline n-Nitrosopyrrrolidine & $0(0.016)$ & 0 (91.9) & \\
\hline Pentachlorobenzene & 74 & 85 & \\
\hline
\end{tabular}


14

Table 4. (continued)

\begin{tabular}{|c|c|c|c|}
\hline Chemical & $\begin{array}{l}\text { WQC for aquatic } \\
\text { organisms and } \\
\text { drinking water }\end{array}$ & $\begin{array}{l}\text { WQC for aquatic } \\
\text { organisms alone } e^{a}\end{array}$ & $\begin{array}{c}\text { WQC for } \\
\text { drinking water } \\
\text { alone }^{b}\end{array}$ \\
\hline Phenol & 300 (org) $^{c}$ & $4,615,385$ & 21,000 \\
\hline \multicolumn{4}{|l|}{ Phthalate esters } \\
\hline Butylbenzyl phthalate & 2,984 & 5,202 & 7,000 \\
\hline Diethylphthalate & 22,631 & 118,019 & 10,500 \\
\hline Dimethylphthalate & 313,000 & $2,900,000$ & \\
\hline Di-n-butylphthalate & 2,715 & 12,100 & 3,500 \\
\hline Bis-2-ethylhexylphthalate & $0(1.8)$ & $0(5.9)$ & $0(2.5)$ \\
\hline $\begin{array}{l}\text { Polychlorinated biphenyls (PCBs) } \\
(1016,1221,1242,1254,1260)\end{array}$ & $0(4.4 \mathrm{E}-05)$ & $0(4.5 \mathrm{E}-05)$ & $0(4.5 \mathrm{E}-03)$ \\
\hline $\begin{array}{l}\text { Polynuclear aromatic } \\
\text { hydrocarbons (PAHs) }\end{array}$ & $0(2.8 \mathrm{E}-03)$ & $0(3.11 \mathrm{E}-02)$ & \\
\hline Pyrene & 956.7 & $10,769.2$ & 1,500 \\
\hline $\begin{array}{l}\text { 2,3,7,8-Tetrachlorodibenzo- } \\
p \text {-dioxin }\end{array}$ & $0(1.3 \mathrm{E}-08)$ & $0(1.4 \mathrm{E}-08)$ & $0(2.2 \mathrm{E}-07)$ \\
\hline 1,2,4,5-Tetrachlorobenzene & 38 & 48 & \\
\hline Tetrachloroethylene & $0(0.8)$ & $0(8.9)$ & \\
\hline Thallium & 13 & 48 & \\
\hline Toluene & $6,764.8$ & 201,294 & 70,000 \\
\hline Toxaphene & $0(7.3 E-04)$ & $0(7.5 \mathrm{E}-04)$ & $0(0.032)$ \\
\hline Trichlorcethylene & $0(2.7)$ & $0(80.7)$ & \\
\hline Trichlorofluoromethane & $0(5.67)$ & $0(470.8)$ & \\
\hline Vinyl chloride & $0(2)$ & $0(525)$ & \\
\hline Zinc & 5,000 (org) $^{c}$ & NA & \\
\hline
\end{tabular}

The criterion value of zero for all potential carcinogens is listed in the table. Concentrations in parentheses for potential carcinogens correspond to a risk of $10^{-6}$.

These adjusted criteria, for drinking water ingestion only, are estimated using a reference dase or carcinogen potency factor and a body weight of $70 \mathrm{~kg}$ and water ingestion rate of $2 \mathrm{~L} / \mathrm{d}$.

"Criteria designated as organoleptic are based on taste and odor effects rather than on human-health effects. Health-based WOC are not available for these chemicals.

${ }^{d}$ Read as $1.3 \times 10^{-4}$.

$\mathbf{N A}=$ not available.

Source: EPA 1991a. EPA Region IV Criteria Charts (October). 
Table 5. Kentucky ambient water quality criteria (WQC) for the protection of human health $(\mu \mathrm{g} / \mathrm{L})^{a}$

\begin{tabular}{|c|c|c|}
\hline Chemical & $\begin{array}{l}\text { WQC for aquatic } \\
\text { organisms and } \\
\text { drinking water }\end{array}$ & $\begin{array}{l}\text { WQC for aquatic } \\
\text { organisms alone }\end{array}$ \\
\hline Acrolein & 320 & 780 \\
\hline Acrylonitrile (C) & $0(5.8 \mathrm{E}-02)^{c}$ & $0(6.5 \mathrm{E}-01)$ \\
\hline Aldrin (C) & $0(7.4 E-05)$ & $0(7.9 \mathrm{E}-05)$ \\
\hline Antimony & 146 & 45,000 \\
\hline Asbestos (C) & $0(30,000$ fibers $/ L)$ & \\
\hline Barium & 1,000 & \\
\hline Benzene (C) & $0(6.6 \mathrm{E}-0 \mathrm{x})$ & $0(40)$ \\
\hline Benzidine (C) & $0(1.2 \mathrm{E}-04)$ & $0(5.3 \mathrm{E}-04)$ \\
\hline Beryllium (C) & $0(6.8 \mathrm{E}-03)$ & $0(1.2 \mathrm{E}-01)$ \\
\hline Cadınium & 10 & 10 \\
\hline Carbon tetrachloride (C) & $0(4.0 \mathrm{E}-01)$ & $0(6.9)$ \\
\hline Chlordane (C) & $0(0.46 \mathrm{E}-03)$ & $0(4.8 \mathrm{E}-04)$ \\
\hline Chloride & 250,000 & NA \\
\hline \multicolumn{3}{|l|}{ Chlorinated benzenes } \\
\hline Monochlorobenzene & 488 & NA \\
\hline Dichlorobenzenes (all isomers) & 400 & 2,600 \\
\hline 1,2,4,5-Tetrachlorobenzene & 38 & 48 \\
\hline Pentachlorobenzene & 74 & 85 \\
\hline Hexachlorobenzene (C) & $0(0.72 \mathrm{E}-03)$ & $0(7.4 \mathrm{E}-04)$ \\
\hline \multicolumn{3}{|l|}{ Chlorinated ethanes } \\
\hline 1,2-Dichloroethane (C) & $0(9.4 \mathrm{E}-01)$ & $0(243)$ \\
\hline 1,1,1-Trichloroethane & 18,400 & $1,030,000$ \\
\hline 1,1,2-Trichloroethane (C) & $0(6.0 \mathrm{E}-01)$ & $0(41.8)$ \\
\hline $1,1,2,2-$ 'retrachloroethane (C) & $0(1.7 \mathrm{E}-01)$ & $0(10.7)$ \\
\hline Hexachloroethane $(C)$ & $0(1.9)$ & $0(8.74)$ \\
\hline \multicolumn{3}{|l|}{ Chlorinated phenols } \\
\hline 2,4-Dichlorophenol & 3,090 & NA \\
\hline 2,4,5-Trichiorophenol & 2,600 & NA \\
\hline 2,4,6-Trichlorophenol (C) & $0(1.2)$ & $0(3.6)$ \\
\hline Pentachlorophenol & 1,000 & NA \\
\hline \multicolumn{3}{|l|}{ Chloroalkyl ethers } \\
\hline bis-(2-Chloroethyl) ether (C) & $0(3.0 \mathrm{E}-02)$ & $0(1.36)$ \\
\hline bis-(2-Chluroisopropyl)ether & 34.7 & 4,360 \\
\hline Chloroform (C) & $0(1.9 \mathrm{E}-01)$ & $0(15.7)$ \\
\hline Chromium (IV) & 50 & NA \\
\hline Chromium (III) & 170,000 & $3,433,000$ \\
\hline Copper & 1,000 & NA \\
\hline
\end{tabular}


16

Table 5 (continued)

\begin{tabular}{|c|c|c|}
\hline Chemical & $\begin{array}{l}\text { WQC for aquatic } \\
\text { organisms and } \\
\text { drinking water }\end{array}$ & $\begin{array}{l}\text { WQC for aquatic } \\
\text { organisms alone }\end{array}$ \\
\hline Cyanide & 200 & NA \\
\hline DDT (C) & $0(2.4 \mathrm{E}-05)$ & $0(2.4 \mathrm{E}-05)$ \\
\hline Dichlorobenzidine (C) & $0(1.0 \mathrm{E}-02)$ & $0(2.0 \mathrm{E}-02)$ \\
\hline \multicolumn{3}{|l|}{ Dichloroethylenes } \\
\hline 1,1-Dichloroethylene (C) & $0(3.3 \mathrm{E}-02)$ & $0(1.85)$ \\
\hline \multicolumn{3}{|c|}{ Dichloropropanes/dichloro-propenes } \\
\hline Dichloropropenes & 87 & 14,100 \\
\hline Dieldrin $(\mathrm{C})$ & $0(7.1 \mathrm{E}-05)$ & $0(7.6 \mathrm{E}-05)$ \\
\hline 2,4-Dinitrotoluene (C) & $0(1.1 \mathrm{E}-01)$ & $0(9.1)$ \\
\hline 1,2-Diphenylhydrazine (C) & $0(4.2 \mathrm{E}-02)$ & $0(5.6 \mathrm{E}-01)$ \\
\hline Endosulfan & 74 & 159 \\
\hline Endrin & 1.0 & NA \\
\hline Ethylbenzene & 1,400 & 3,280 \\
\hline Fluoride & 1,000 & NA \\
\hline Fluoranthene & 42 & 54 \\
\hline Halomethanes (C) & $0(1.9 \mathrm{E}-01)$ & $0(15.7)$ \\
\hline Heptachlor (C) & $0(0.28 \mathrm{E}-03)$ & $0(2.9 \mathrm{E}-04)$ \\
\hline Hexachlorobutadiene (C) & $0(4.5 \mathrm{E}-01)$ & $0(50)$ \\
\hline \multicolumn{3}{|l|}{ Hexachlorocyclohexanes $(\mathrm{HCCH})$} \\
\hline Alpha-HCCH (C) & $0(9.0 \mathrm{E}-03)$ & $0(3.1 \mathrm{E}-102)$ \\
\hline Beta-HCCH (C) & $0(1.6 \mathrm{E}-02)$ & $0(5.5 \mathrm{E}-02)$ \\
\hline Gamma-HCCH (C) & $0(1.9 \mathrm{E}-02)$ & $0(62.5 \mathrm{E}-03)$ \\
\hline Technical-HCCH (C) & $0(1.2 \mathrm{E}-02)$ & $0(41.4 \mathrm{E}-03)$ \\
\hline Hexachlorocyclopentadiene & 206 & NA \\
\hline Isophorone & 5,200 & 520,000 \\
\hline Lead & 50 & NA \\
\hline Manganese & 50 & NA \\
\hline Mercury & 144 & $14.6 \mathrm{E}-02$ \\
\hline Nickel & 13.4 & 100 \\
\hline Nitrates & 10,000 & NA \\
\hline Nitrobenzene & 19,800 & NA \\
\hline \multicolumn{3}{|l|}{ Nitrophenols } \\
\hline 2,4-Dinitro-o-cresols & 13.4 & 765 \\
\hline Dinitrophenol & 70 & 14,300 \\
\hline \multicolumn{3}{|l|}{ Nitrosamines } \\
\hline n-Nitrosodimethylamine $(C)$ & $0(1.4 \mathrm{E}-03)$ & $0(16)$ \\
\hline$n$-Nitrosodiethylamine (C) & $0(0.8 \mathrm{E}-03)$ & $0(1.24)$ \\
\hline n-Nitrosodi-n-butylamine (C) & $0(6.4 \mathrm{E}-03)$ & $0(5.9 \mathrm{E}-01)$ \\
\hline
\end{tabular}


17

Table 5 (continued)

\begin{tabular}{lll}
\hline \multicolumn{1}{c}{ Chemical } & $\begin{array}{l}\text { WQC for aquatic } \\
\text { organisms and } \\
\text { drinking water }\end{array}$ & \multicolumn{1}{c}{$\begin{array}{l}\text { WQC for aquatic } \\
\text { organisms alone }\end{array}$} \\
\hline$n$-Nitrosodiphenylamine (C) & $0(4.9)$ & $0(16)$ \\
$n$-Nitrosopyrrolidine (C) & $0(1.6 \mathrm{E}-02)$ & $0(92)$ \\
Pentachlorophenol & 1,000 & NA \\
Phenol & 3,500 & NA \\
Phthalate esters & & \\
Dimethylphthalate & 313,000 & $2,900,000$ \\
Diethylphthalate & 350,000 & $1,800,000$ \\
Dibutylphthalate & 34,0001 & 54,000 \\
Di-2-ethylhexylphthalate & 15,000 & 50,000 \\
Polychlorinated biphenyls (C) & $0(7.9 \mathrm{E}-05)$ & $0(7.9 \mathrm{E}-05)$ \\
Polynuclear aromatic hydrocarbons (C) & $0(2.8 \mathrm{E}-03)$ & $0(31.1 \mathrm{E}-03)$ \\
Selenium & 10 & $\mathrm{NA}$ \\
Silver & 50 & $\mathrm{NA}$ \\
Sulfates and chlorides & 250,000 & $\mathrm{NA}$ \\
2,3,7,8-Tetrachlorodibenzo-p-dioxin (C) & $0(1.3 \mathrm{E}-08)$ & $0(1.4 \mathrm{E}-08)$ \\
Tetrachloroethylene (C) & $0(8.0 \mathrm{E}-01)$ & $0(8.85)$ \\
Thallium & 13 & 48 \\
Toluene & 14,300 & 424,000 \\
Toxaphene (C) & $0(7.1 \mathrm{E}-04)$ & $0(0.73 \mathrm{E}-03)$ \\
Trichloroethylene (C) & $0(2.7)$ & $0(80.7)$ \\
Vinyl chloride (C) & $0(2.0)$ & $0(525)$ \\
\hline
\end{tabular}

aAll potential carcinogens are noted with a (C) in the list of chemicals. The EPA criterion value of zero for all potential carcinogens is listed in the table. Concentrations in parentheses for potential carcinogens correspond to a risk of $10^{-6}$. Kentucky also uses a risk of $10^{-6}$ for potential carcinogens.

${ }^{b}$ Kentucky Water Quality Regulations. Kentucky Administrative Record Title 401, Chapter 5:031 (Surface Water Standards).

'Read as $5.8 \times 10^{-2}$.

$\mathrm{NA}=$ not available.

However, because the EPA Region IV WQC have been calculated based on the most current scientific information, they may be considered relevant and appropriate to cleanup at PGDP. The NCP states that if two ARARs are available for a chemical, the most stringent applies [40 CFR 300.400 $(\mathrm{g})(4)]$. In addition, CERCLA stipulates that WQC must be based on the most current scientific information [CERCLA \$121(d)(2)(B)]. The use of the appropriate WQC for cleanup at PGDP will depend on the use classification of the stream in question and the scientific rationale for calculation of the WQC; these will be analyzed on a sitespecific basis. When the Kentucky Office of Water Quality revises its current WQC, this table will be revised accordingly.

The Kentucky regulations list six use-designation categories for Kentucky's surface waters and groundwaters (Title 401 KAR 5:026). Specific water quality standards are promulgated 
for each use category. Kentucky has classified the Ohio River in McCracken and Ballurd counties for domestic water supply, warm water aquatic habitat, and primary and secondary contact recreation (401 KAR 5:026). In addition, ceriain portions of the Ohio River are designated as outstanding resource waters (see Sect. 4.6). The Kentucky standards also state that "all other surface waters which have not been specifically listed for use classifications shall be classified for domestic water supply, warm water aquatic habitat, and primary and secondary contact recreation" (401 KAR 5:026). Little and Big Bayou Creeks would therefore have these use classifications by reference.

Table 6 lists federal WQC for the protection of freshwater aquatic life. When the designated use classification requires protection of aquatic life or when environmental factors are being considered at a remedial action site, WQC for the protection of aquatic life that are more stringent than the SDWA MCL may be relevant and appropriate (55 FR 8754) for CERCLA cleanup. The Kentucky WQC for a warm water habitat are found in Title 401 KAR 5:031 (Table 2 of Kentucky surtace water standards); these criteria are identical to the federal WQC.

\section{AIR}

Primary National Ambient Air Quality Standards (NAAQS) for six chemicals appear in 40 CFR 50; these standards are based on the direct health effects of those chemicals to sensitive groups, with no economic factors considered. The NAAQS take into consideration all sources of exposure to a given chemical and establish ceilings that are not to be exceeded in the United States. Only major new sources, or major modifications to existing sources, must attain NAAQS. Although PGDP is in a Kentucky "attainment area," it is unlikely that any emissions from remediation at PGDP will be considered a "major" source.

NAAQS are established as the criteria state and local governments must pian to achieve and thus are not directly enforceable. Under $\$ 110$ of the CAA, states are required to implement regulations to achieve the NAAQS. The ambient air quality standards appear in Title 401, KAR Chapter 53:010, Appendix A. The Kentucky state air emission standards are considered "hybrid" ARARs and will be summarized as action-specific ARARs in Sect. 5.2.

National Emission Standards for Hazardous Air Pollutants (NESHA.Ps) for various industrial sources that emit one of several pollutants are established in 40 CFR 61. Most of the NESHAPs are generally neither applicable nor relevant and appropriate to cleanup at CERCLA sites because they regulate particular types of sources that would not be expected to be found at a CERCLA site. However, the NESHAPs will be considered as potential "hybrid" ARARs on a site-specific basis during the selection of remedial alternatives; in particular, the NESHAPs for radionuclides will be reviewed (see Sect. 3.1).

The Clean Air Act Amendments of 1990 establish a list of 189 additional hazardous air pollutants for which technology-based standards must be promulgated. These standards will be applicable to categories of emissions rather than to the substances emitted and will be analyzed when proposed. 
Table a. Federal ambient water quality criteria for the protection of ff sliwater organisms

\begin{tabular}{|c|c|c|}
\hline Chemical & $\begin{array}{c}\text { Maximum } \\
(\mu g / L)^{a}\end{array}$ & $\begin{array}{c}24-h \\
(\mu g /)^{b}\end{array}$ \\
\hline Aldrin & 3 & NA \\
\hline Aluminum & $750^{\circ}$ & $87^{\circ}$ \\
\hline Ammonia & \multicolumn{2}{|c|}{ Concentrations are temperature and $\mathrm{pH}$ dependent } \\
\hline Arsenic (III) & 360 & 190 \\
\hline Cadmium & $1.8^{i}$ & $0.66^{d}$ \\
\hline Chlordane & 2.4 & $4.3 \mathrm{E}-03^{\mathrm{es}}$ \\
\hline Chloride & $860,0 \times \times$ & 230,000 \\
\hline Chlorine & 19 & 11 \\
\hline Chlorpyrifos & 0.083 & 0.041 \\
\hline Chromium (VI) & 16 & 11 \\
\hline (III) & $984^{d}$ & $117^{d}$ \\
\hline Copper & $9.22^{d}$ & $6.54^{d}$ \\
\hline Cyanide & 22 & 5.2 \\
\hline Dichlorodiphenyl-trichloroethane (DDT) & 1.1 & $1.0 \mathrm{E}-038$ \\
\hline Demeton & NA & 0.1 \\
\hline Dieldrin & 2.5 & $1.9 \mathrm{E}-03$ \\
\hline Endosulfan & 0.22 & 0.056 \\
\hline Endrin & 0.18 & $2.3 E-0.3 f$ \\
\hline Guthion & N.A & 0.01 \\
\hline Heptachlor & 0.52 & $3.8 \mathrm{E} \backsim 03^{f}$ \\
\hline Heptachlor epoxide & 0.52 & $3.8 \mathrm{E}-0.3 f$ \\
\hline $\begin{array}{l}\text { Hexachlorocyclohexanes (HCCH) } \\
\text { gamma-HCCH (Lindane) }\end{array}$ & 2 & 0.08 \\
\hline Hydrogen sultide & NA & 2 \\
\hline Iron & NA & 1,000 \\
\hline Lead & $33.8^{d}$ & $1.32^{d}$ \\
\hline Malathion & NA & 0.1 \\
\hline Mercury & 2.4 & $0.012^{\prime}$ \\
\hline Methoxychlor & NA & 0.03 \\
\hline Mirex & NA & $1.0 \mathrm{E}-103$ \\
\hline Nickel & $789^{d}$ & $88^{d}$ \\
\hline Parathion & 0.065 & 0.013 \\
\hline Pencachlorophenol & $3.32^{h}$ & $2.1^{h}$ \\
\hline $\mathrm{pH}$ & NA & $6.5-9$ \\
\hline Polychlorinatel biphenyls & NA & 0.014 \\
\hline Selenium (inorganic selenite) & 20 & 5 \\
\hline Silver & $1.23^{d}$ & NA \\
\hline Sulfides & NA & 2 \\
\hline Zinc & $65^{d}$ & $59^{d}$ \\
\hline
\end{tabular}

anc-bour average concentration not to be exceeded more than once every 3 years.

"Four-day average concentration not to be excecded more than once every 3 years.

cpH 6.5-9.0.

Water hardiness depentent criteria ( $50 \mathrm{mg} / \mathrm{L}$ as $\mathrm{CaCO}_{3}$ ).

"Read pas $4.3 \times 10^{-3}$.

flased on marketability of fish.

trinal residue value based on wildlife feeding stwdy.

"pHidepardent criteria.

Sourc: EPA 1991 1. EPA Region IV Criteria Chart (October). 


\section{$23 \mathrm{SOIL}$}

Very little legislation or guidance is available governing cleanup criteria for contaminated soils at CERCLA sites. Land disposal of treated hazardous wastes is addressed in RCRA in the form of land disposal restrictions (LDR) (40 CFR 268), and these are addressed as "hybrid" ARARs in Chapter 5. If a site is identified as a RCRA Sect. 3004(u) SWMU, or if RCRA characteristic or hazardous waste as defined in 40 CFR 261 is present at the site, it is subject to RCRA corrective action regulations. The proposed RCRA corrective action regulations address risk-based cleanup standards for soils (55 FR 30798); these are briefly discussed in Sect. 5.1.3. Treatment standards for contaminated soil and debris at CERCLA sites are discussed in Sect. 5.1.1.

The regulations found in the Toxic Substances Control Act (TSCA) contain storage, disposal, and cleanup requirements for materials contaminated with polychlorinated biphenyls (PCBs). These regulations limit concentrations of PCBs disposed of in soil to $50 \mathrm{ppm}$ and require that any soils containing concentrations of $\mathrm{PCBs}$ greater than $50 \mathrm{ppm}$ (40 CFR 761.60) be incinerated (40 CFR 761.70) or stored in a chemical waste landfill ( 10 CFR 761.75). Requirements that must be achieved for PCBs disposed of by incineration (40 CFR 761.70), in a chernical waste landfill (40 CFR 761.75), or by other disposal methods [40 CFR 761.60(a)(5)(iii)] are also specified in TSCA.

\section{OTHER "TO-BE-CONSIDERED" (TBC) GUIDANCE}

EPA has suggested cleanup values for lead in soils based on studies of lead levels in blood of exposed children. The EPA Office of Solid Waste and Emergency Response (OSWER) has issued a directive (Directive 9355.4-02, dated September 7, 1989) which recommends a cleanup level for soils of 500 to $1000 \mathrm{ppm}$ lead.

Although not an ARAR, EPA has published a TSCA PCB spill cleanup policy (52 FR 10688) which recommends cleanup standards for PCBs of $25 \mathrm{ppm}$ for sites with restricted access; a 10-ppm cleanup level is recommended for residential and unrestricted access rural areas. In this latter case, a 10-in. cap of clean soil must cover the site. In the EPA guidance report for remedial actions at Superfund sites containing PCBs, preliminary remediation goals are set at $1 \mathrm{ppm}$ for residential land use (a risk of $10^{-5}$ ) and between $10 \mathrm{ppm}$ and $25 \mathrm{ppm}$ for industrial and/or remote areas (a risk of 10 $0^{-4}$ ) (EPA 1990). Remedial alternatives should reduce concentrations to these levels or limit exposures. EPA also presents an approach to deriving cleanup levels of PCBs in sediments (EPA 1990). This approach results in "sediment quality criteria" as a function of organic carbon concentrations and is meant to protect wildlife that consumes freshwater benthic species. These values are considered TBC guidance, not ARARs.

In addition, the Kentucky Division of Waste Management uses an internal guidance value of $1 \mathrm{ppm}, 0.1 \mathrm{ppm}$, and 65 parts per trillion for cleanup of PCBs in soils, sediments, and water, respectively (Taylor 1991). This value has no regulatory or statutory basis, but may be considered as TBC guidance for cleanup of PCBs at PGDP.

In the absence of federally or state-promulgated ARARs or in the case where ARARs are not adequately protective, EPA states a preference for Office of Drinking Water (ODW) 
Health Advisories (HAs), RfDs or Reference Concentrations (RfCs) for systemic toxicants, and CSFs for carcinogens (EPA 1988).

The EPA ODW has developed nonregulatory HAs for concentrations of noncarcinogenic contaminants in drinking water at which no adverse health effects would be expected to occur. Table 7 lists 1-d, $: 0-d$, and longer-term (several months to several years) HAs for a child weighing $10 \mathrm{~kg}$. Thesi advisories have been developed as guidance values for short-term exposure situations such as spills or accidents and are not intended for use in estimating acceptable lifetime intakes (50 FR 46936). Longer-term and lifetime advisory levels for a $70-\mathrm{kg}$ adult are also listed in Table 7.

EPA uses the lifetime HA (for noncarcinogens only) to develop MCLs and MCLGs; HAs will most likely represent future $\mathrm{MCL}$ proposals. However, these values assume that $20 \%$ of a person's exposure to a compound is via the drinking water pathway. Therefore, if site-specific information indicates that there are no other sources of exposure to a particular compound, the lifetime HA may be increased by a factor of 5 . This will be considered as sitespecific exposure pathways are developed. The EPA ODW has also determined the concentration of specific carcinogens in drinking water that will result in one excess cancer in one million people (a risk of $10^{-6}$ ) following a lifetime exposure. Although not HAs, which are only developed for noncarcinogens, the carcinogenic values are listed in Table 7 as they were developed by the ODW.

EPA has also developed other TBC guidance values in the form of RfDs, RfCs, and CSFs which are available through IRIS (EPA 1991b) and the EPA Health Effects Assessment Summary Tables (EPA 1991c). The information found in IRIS is frequently in flux, as it is constantly undergoing EPA review and verification. Therefore, chemical-specific values are not listed here. 


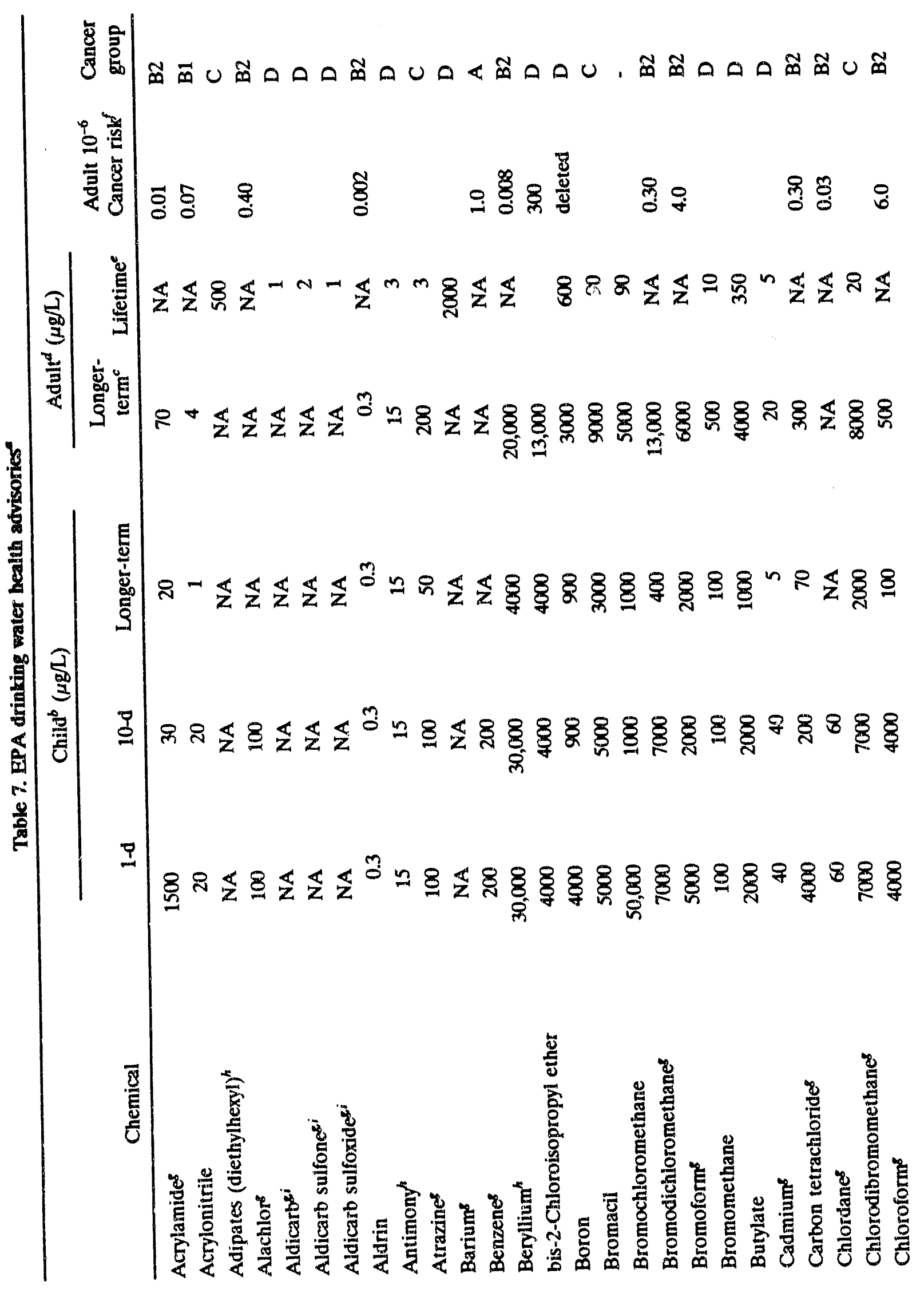




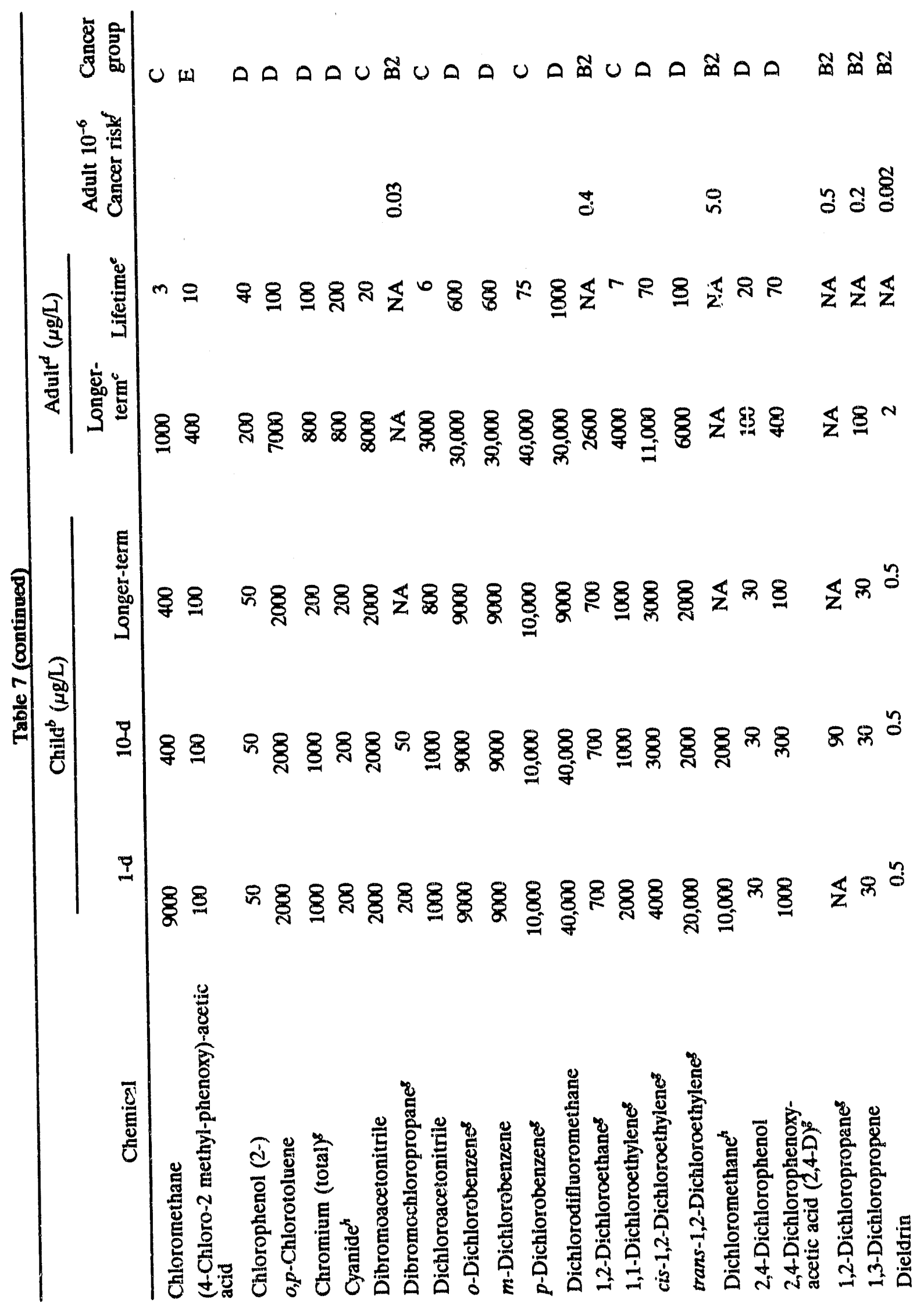




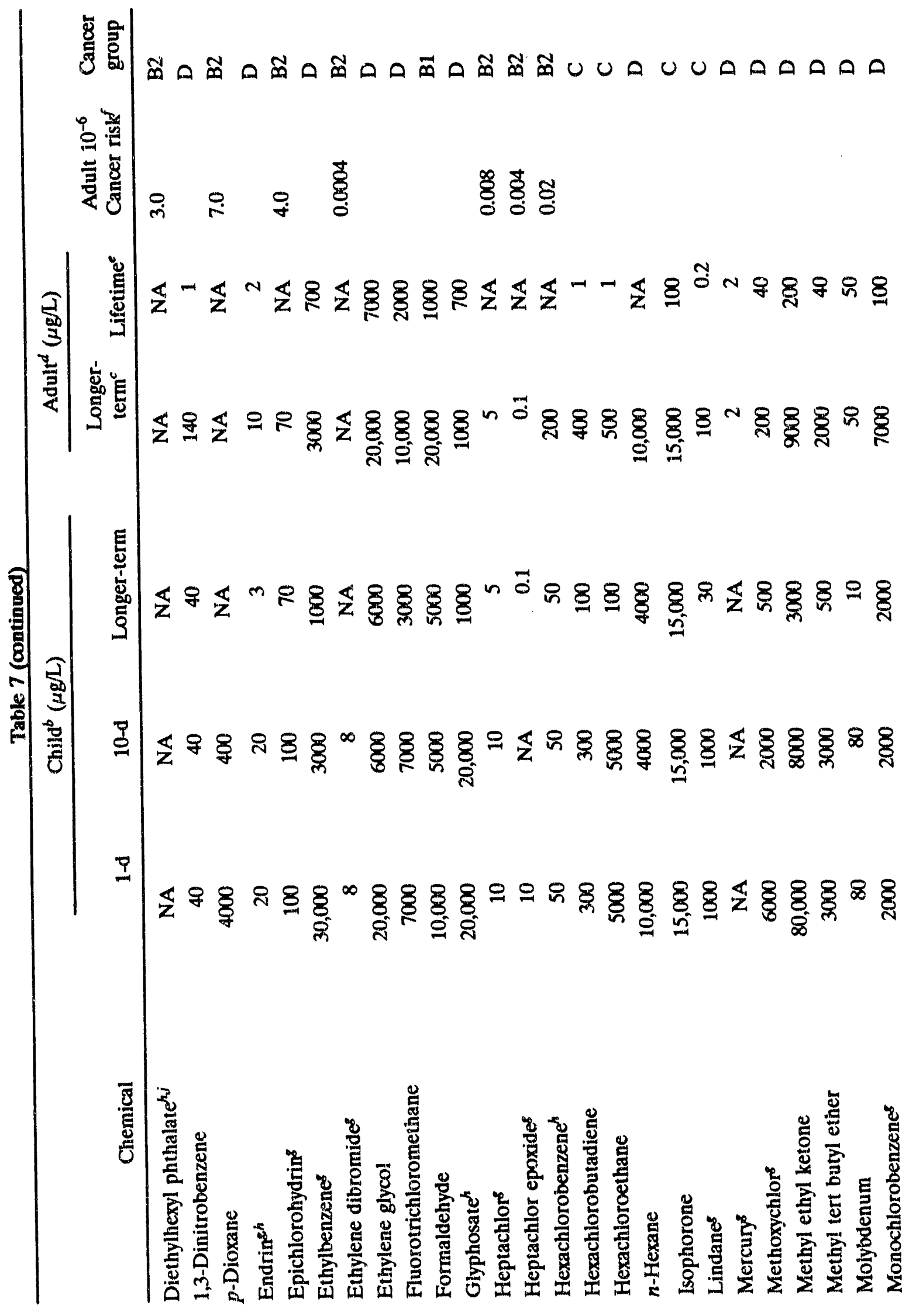




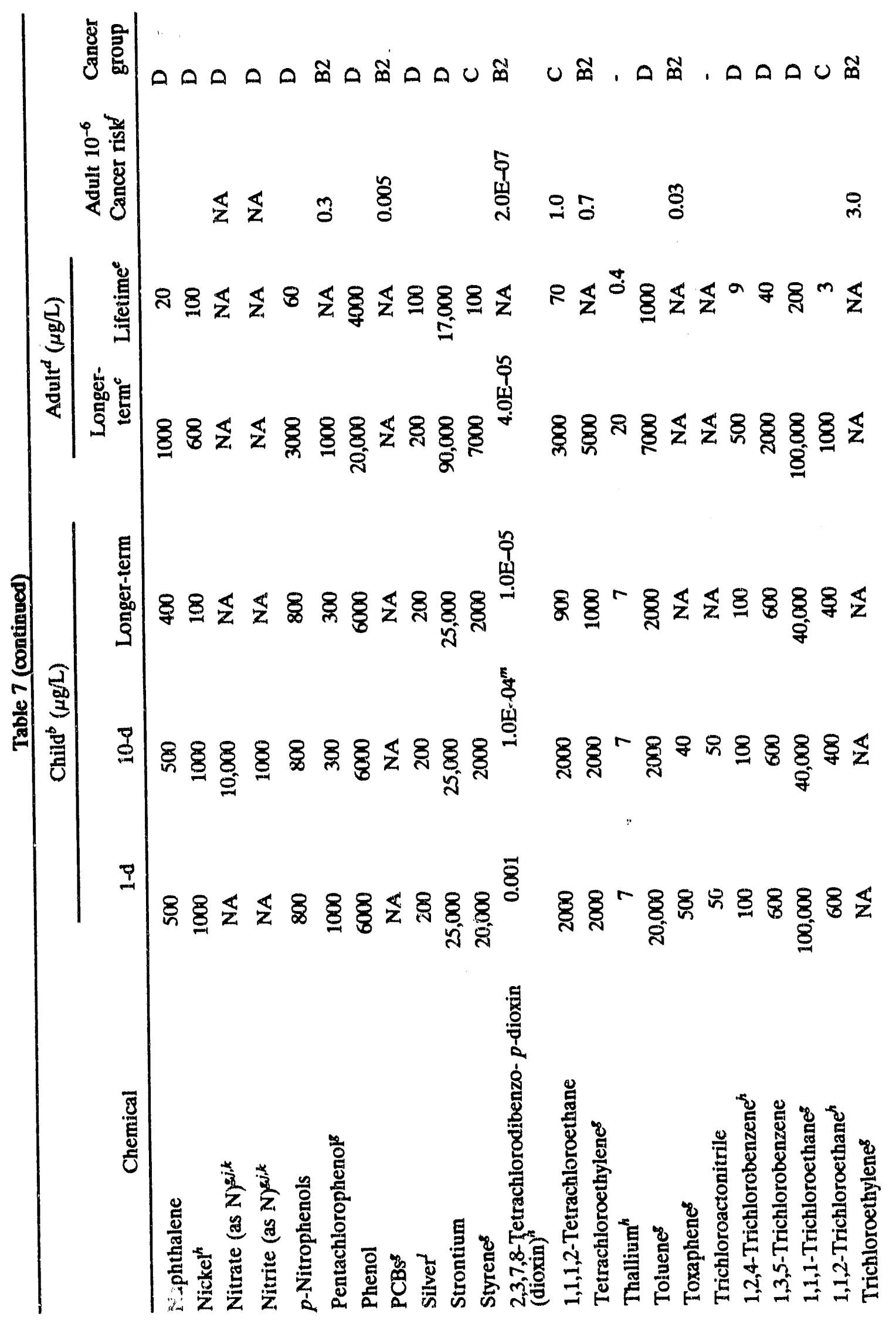




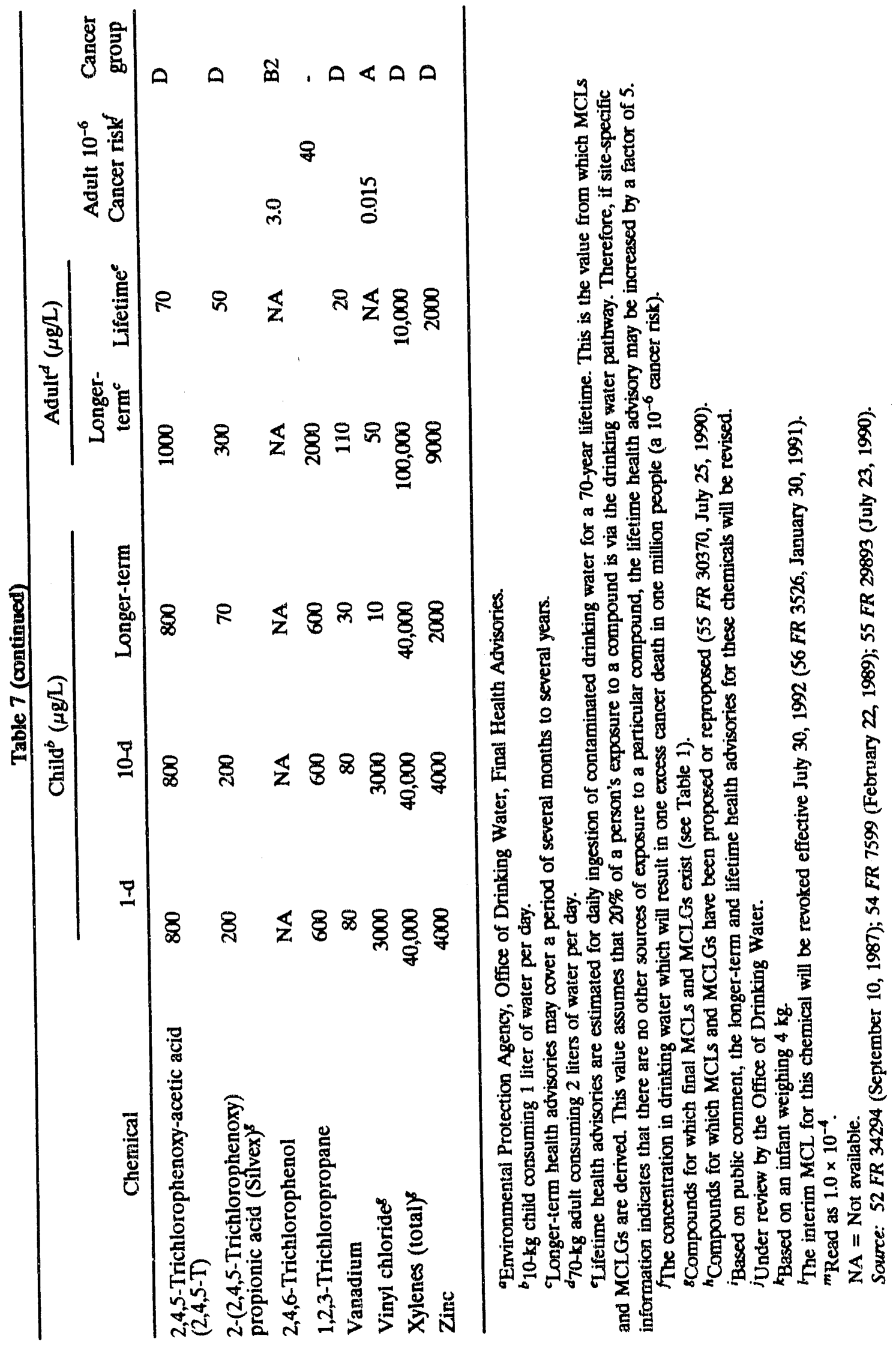




\section{RADIATION PROTECTION STANDARDS}

Very few applicable standards are available for the cleanup of radioactively contaminated CERCLA sites. The Atomic Energy Act (AEA) of 1954 and its amendments delegated authority for control of nuclear energy to DOE, the U.S. Nuclear Regulatory Commission (NRC), and EPA. In addition, certain states have regulatory authority and programs for radioactive waste. EPA's regulations are derived from several other statutes as well and cover many types of activities and all types of radioactive materials. The NRC licenses the possession and use of various types of radioactive materials at certain types of facilities. Kentucky is an NRC-agreement state and, as such, has its own authority and licensing regulations.

DOE is authorized to control all types of nuclear materials at sites under its jurisdiction and is exempt from the NRC licensing and regulatory requirements. Therefore, NRC regulations are not considered to be ARARs for CERCLA cleanup at DOE facilities; however, a summary of the general content of NRC regulations will be presented here. DOE regulations for handling and cleanup of radioactive materials are outlined in a series of internal DOE orders that are legally binding to DOE contractors but are not considered by EPA to be ARARs. Therefore, for the purposes of development of ARARs, DOE orders will be treated, along with the NRC regulations, as TBC guidance.

Radionuclide contamination has been detected in groundwater, surface water, soils, and sediments; low-level radioactive waste as well as transuranic (TRU) waste may be present at PGDP. The proper definition of "mixed low-level radioactive and hazardous waste" has caused considerable debate with regard to dual jurisdiction by EPA and NRC. However, EPA has published a clarification of the problem $(53 F R 37045$, September 23,1988$)$, as did DOE previously [52 FR 15937, May 1, 1987 and DOE Order 5400.3 (Hazardous and Radioactive Mixed Waste Program, dated February 22, 1989)]. In effect, mixed wastes are those containing a RCRA hazardous waste as defined in 40 CFR 261 and a radioactive waste subject to the AEA. RCRA regulations apply to the hazardous component of the waste, and AEA regulations apply to the radioactive component. When the application of both standards is conflicting or inconsistent, RCRA yields to the AEA. Kentucky received final authorization to regulate radioactive mixed waste on December 19, 1988 (53 FR 41164, October 20,1988); however, the state has not implemented any regulations governing the radioactive component of mixed waste (Jeffs 1991).

\subsection{EPA REGULATIONS}

EPA has promulgated MCLs for radionuclides in community water systems (see Table 8). These MCLs appear in two forms-concentration limits for certain alpha-emitting radionuclides (40 CFR 141.15) and an annual dose limit for the ingestion of certain beta- and gamma-emitting radionuclides (40 CFR 141.16). Kentucky lists identical MCLs in the Kentucky Public and Semipublic Drinking Water Regulations, Title 401 KAR Chapter 8:550, Sect. 4.

MCLs and MCLGs were proposed for radon and uranium and reproposed for ${ }^{226} \mathrm{Ra}$ and ${ }^{228} \mathrm{Ra}$ and beta emitters and photon emitters on July 18,1991 , and are included in Table 8. 
Table 8. Radionuclide-specific ARARs for groundwater and surface water contamination at the Paducah Gaseous Diffusion Plant

\begin{tabular}{lll}
\hline \multicolumn{1}{c}{ Radionuclide } & \multicolumn{1}{c}{ SDWA MCLs } & $\begin{array}{c}\text { Proposed SDWA } \\
\mathrm{MCLs}^{b}\end{array}$ \\
\hline Radium $^{c}$ & $5 \mathrm{pCi} / \mathrm{L}$ & $20 \mathrm{pCi} / \mathrm{L}$ \\
Gross alpha $^{d}$ & $15 \mathrm{pCi} / \mathrm{L}$ & $15 \mathrm{pCi} / \mathrm{L}$ \\
Gross beta $_{\text {Natural uranium }}$ & $4 \mathrm{mrem} / \mathrm{year}$ & $4 \mathrm{mrem} / \mathrm{year}$ \\
Radon-222 & & $20 \mu \mathrm{g} / \mathrm{L}^{e}$ \\
Strontium-90 & & $300 \mathrm{pCi} / \mathrm{L}$ \\
Tritium & $8 \mathrm{pCi} / \mathrm{L}$ & $8 \mathrm{pCi} / \mathrm{L}$ \\
All other manmade radionuclides & $20,000 \mathrm{mCi} / \mathrm{L}$ & $20,000 \mathrm{pCi} / \mathrm{L}$ \\
& $4 \mathrm{mrem} / \mathrm{year}^{f}$ & $4 \mathrm{mrem} / \mathrm{year}$ \\
& & \\
\hline
\end{tabular}

${ }^{a}$ SDWA MCL $=$ Safe Drinking Water Act maximum contaminant level; $40 \mathrm{CFR}$ 141.15 and 141.16 and 401 KAR 8:550, Sect. 4.

${ }^{b}$ Proposed rule, July 18, 1991 (56 FR 33050); final rule expected April 1993.

The present MCL applies to combined ${ }^{26} \mathrm{Ra}$ and ${ }^{288} \mathrm{Ra}$; the proposed MCL applies to each separately.

${ }^{d}$ The present MCL excludes radon and uranium but includes ${ }^{226} \mathrm{Ra}$; the proposed MCL excludes all three radionuclides.

eApproximately equal to $30 \mathrm{pCi} / \mathrm{L}$.

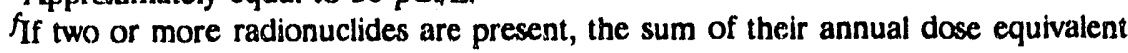
to the total body or to any organ shall not exceed $4 \mathrm{mrem} / \mathrm{year}$.

Final promulgation of the concentration limits is not expected until April 1993. As with the chemical-specific MCLs, these may be relevant and appropriate for cleanup of contaminated groundwater at PGDP. Table 9 lists EPA, NRC, and DOE radiation protection standards that are described below.

Subpart H of 40 CFR 61 addresses atmospheric radionuclide emissions from DOE facilities and may be applicable to airborne emissions during cleanup of PGDP. EPA has issued a final NESHAP rule $(54$ FR 51654 , December 15,1989$)$ that limits emissions of radionuclides to the ambient air from DOE facilities to amounts that would not cause any member of the public to receive an effective dose equivalent of 10 mrem/year (40 CFR 61.92).

Environmental protection standards for the management, storage, and disposal of spent nuclear fuel, high-level wastes, and TRU wastes are found in 40 CFR 191. Management and storage shall be conducted so as to provide a reasonable assurance that no member of the public in the general environment shall receive a combined annual dose equivalent of greater than $25 \mathrm{mrem}$ to the whole body and $75 \mathrm{mrem}$ to any organ (40 CFR 191.03). Disposal systems for the wastes defined above must be designed "to provide a reasionable expectation that, for 1000 years after disposal, undisturbed performance of the disposal system shall not cause the annual dose equivalent from the disposal system to any member of the public in the accessible environment to exceed $25 \mathrm{mrem}$ to the whole body and $75 \mathrm{mrem}$ to any critical organ." All potential pathways must be considered (40 CFR 191.15). These standards may be relevant and appropriate for the storage or disposal of radioactive materials at PGDP. In 40 CFR 191.04 alternative standards are established for DOE facilities when EPA determines that such standards prevent any member of the public from receiving a continuous exposure 
Table 9. Radiation protection standards that may be ARAR for the Paducah Gaseous Diffusion Plant

\begin{tabular}{|c|c|c|c|}
\hline Regulation & Applicability & Exposure conditions & Standard \\
\hline 40 CFR 61 & $\begin{array}{l}\text { NESHAPs }{ }^{b} \text { for DOE } \\
\text { facilities }\end{array}$ & $\begin{array}{l}\text { Public exposure, } \\
\text { airborn emissions }\end{array}$ & 10 mrem/year \\
\hline 40 CFR 141 & $\begin{array}{l}\text { Drinking water maximum } \\
\text { contaminant levels }\end{array}$ & $\begin{array}{l}\text { Community water } \\
\text { systems, gross beta }\end{array}$ & 4 mrem/year \\
\hline 40 CFR 191 & $\begin{array}{l}\text { Spent nuclear fuel, } \\
\text { high-level and transuranic } \\
\text { wastes }\end{array}$ & $\begin{array}{l}\text { Public exposure, } \\
\text { all sources }\end{array}$ & $\begin{array}{l}25 \text { mrem/year } \\
\text { (total body) } \\
75 \text { mrem/year } \\
\text { (thyroid) }\end{array}$ \\
\hline \multirow[t]{2}{*}{10 CFR 20} & $\begin{array}{l}\text { Radiation from facilities } \\
\text { licensed by the Nuclear } \\
\text { Regulatory Commission }\end{array}$ & $\begin{array}{l}\text { Worker exposure } \\
\text { dimits in unrestricted } \\
\text { areas }\end{array}$ & 5 rem/year \\
\hline & & $\begin{array}{l}\text { Public exposure, } \\
\text { unrestricted areas }\end{array}$ & 100 mrem/year \\
\hline 10 CFR 61 & $\begin{array}{l}\text { Licensing requirements } \\
\text { for land disposal of } \\
\text { radioactive waste }\end{array}$ & $\begin{array}{l}\text { Public exposure, all } \\
\text { sources }\end{array}$ & $\begin{array}{l}25 \text { mrem/year } \\
\text { (total body) } \\
75 \text { mrem/year } \\
\text { (thyroid) }\end{array}$ \\
\hline \multirow[t]{3}{*}{ DOE Order 5400.5} & $\begin{array}{l}\text { Radiation Protection of } \\
\text { the Public and the } \\
\text { Environment }\end{array}$ & $\begin{array}{l}\text { Public exposure, all } \\
\text { sources }\end{array}$ & 100 mrem/year \\
\hline & & Single acute exposure & $500 \mathrm{mrem} / \mathrm{year}$ \\
\hline & & $\begin{array}{l}\text { Aquatic organism } \\
\text { exposure, absorbed } \\
\text { dose }\end{array}$ & $1 \mathrm{rad} / \mathrm{d}$ \\
\hline \multirow[t]{2}{*}{ DOE Order $5820.2 \mathrm{~A}$} & $\begin{array}{l}\text { Radioactive Waste } \\
\text { Management }\end{array}$ & $\begin{array}{l}\text { Public exposure, all } \\
\text { sources, excluding air }\end{array}$ & 25 mrem/year \\
\hline & & $\begin{array}{l}\text { Public exposure, } \\
\text { atmospheric releases }\end{array}$ & $10 \mathrm{mrem} / \mathrm{year}$ \\
\hline \multirow[t]{3}{*}{ DOE Order 5480.11} & $\begin{array}{l}\text { Radiation Protection for } \\
\text { Occupational Workers }\end{array}$ & $\begin{array}{l}\text { Worker exposure } \\
\text { limits, continuous } \\
\text { exposure }\end{array}$ & $\begin{array}{l}5 \text { mrem/year, } \\
\text { cancer effects }\end{array}$ \\
\hline & & & $\begin{array}{l}50 \text { mrem/year, } \\
\text { noncancer } \\
\text { effects }^{d}\end{array}$ \\
\hline & & $\begin{array}{l}\text { Public exposures, } \\
\text { controlled areas }\end{array}$ & 100 mrem/year \\
\hline
\end{tabular}

${ }^{a} \mathrm{ARAR}=$ applicable or relevant and appropriate requirement

${ }^{b}$ NESHAPs = National Emission Standards for Hazardous Air Pollutanis

${ }^{c}$ Final rule of May 22, 1991 ( 56 FR 23360), effective June 21, 1991. Implementation of this regulation by NRC licensees may be deferred until January 1, 1993. This rule deleted the quarterly limits for occupational exposures and lowered the limit for public exposure from 500 mrem/year to 100 mrem/year.

${ }^{d}$ Proposed as 10 CFR 835 (56 FR 64334, December 9, 1991) 
of more than 100 mrem/year effective dose equivalent or an infrequent exposure of more than 500 mrem/year effective dose equivalent from all sources, excluding natural background and medical exposures. However, EPA plans to revise its standards and promulgate generally applicable environmental standards to establish limits for the release of radioactive materials to the environment (NPRM, May 1992; final rule, June 1993). Whether this proposed rule will contain the alternative standards clause is unknown. When promulgated, these standards will supersede those mentioned above as potential ARARs.

EPA intends to develop environmental radiation protection standards for the disposal of low-level waste (LLW) (possibly including naturally occurring and accelerator-produced radioactive material) under $40 \mathrm{CFR} 193$ and 764 . The intent of these standards will be to protect the public health and general environment from potential adverse effects from LLW disposal. These proposed regulations may provide TBC guidance for cleanup of PGDP and, when promulgated, will be considered as ARARs. This proposed rule is currently on hold, however, pending resolution of the issue with the NRC (Houlberg et al. 1991).

In addition, EPA is developing public health and environmental radiation protection criteria for cleanup of residual radioactive materials at decommissioned DOE, Department of Defense, and NRC-licensed sites, as well as other sites. These criteria may provide useful TBC guidance for remedial response at PGDP. However, this rulemaking initiative is also on hold. (Houlberg et al. 1991).

\subsection{NRC REGULATIONS}

As mentioned previously, DOE is not regulated by the NRC; however, NRC regulations might provide some TBC guidance for cleanup of radioactive waste at PGDP. Thus the regulations are summarized here. The standards for protection against radiation (10 CFR 20) were revised recently (56 FR 23360, May 21, 1991). They are designed to limit radiation exposures from NRC-licensed activities. They provide permissible worker exposure limits for restricted areas of 5 rem/year (10 CFR 20.1201) and lowered radiation exposure limits to the general public in unrestricted areas from 500 to 100 mrem/year (10 CFR 20.1301).

Appendix B of 10 CFR 20.1001-20.2401 sets annual average concentration limits for radionuclides in airborne and liquid effluents. These represent the radionuclide concentrations which, if inhaled or ingested, would produce a total annual effective dose equivalent of 50 mrern.

The Kentucky Radiation Control Branch has promulgated licensing regulations that are almost entirely the same as the 10 CFR 20 regulations (902 KAR 100 et seq), so they will not be repeated here. These regulations are currently under revision to incorporate the changes in the 10 CFR 20 regulations described above, with promulgation anticipated for late 1992 (Jeffs 1991).

The NRC has promulgated licensing requirements for land disposal of radioactive waste (10 CFR 61). Part 61 contains procedural requirements and performance standards applicable to any method of land disposal, with specific technical requirements for near-surface disposal of radioactive waste (see Table 10). Although not an ARAR, the substantive requirements found in this regulation might provide TBC guidance for disposal options selected at PGDP. 


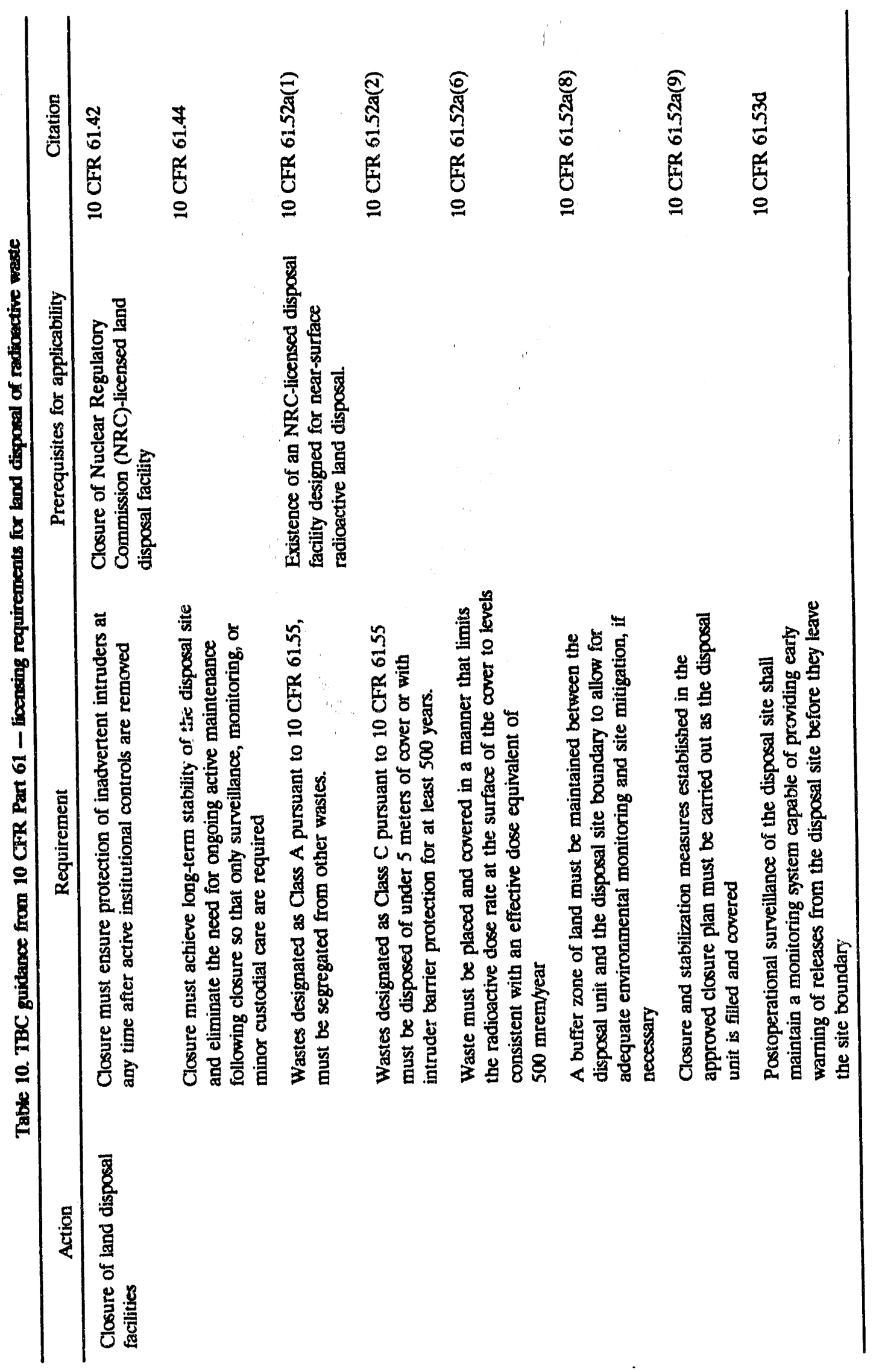


According to 10 CFR 61.41, concentrations of radioactive materials released to the environment in all media must not result in an annual dose exceeding $75 \mathrm{mrem}$ tc the thyroid and 25 mrem to total body or all other organs of any member of the public (Table 9). In addition, reasonable effort must be made to maintain releases of radioactive materials to "as low as reasonably achievable" (ALARA). It is stated in 10 CFR 61.42 that inadvertent intruders must be protected following cessation of active institutional controls, and 10 CFR 61.41 provides that operations at land disposal facilities must be carried out in compliance with 10 CFR 20.

\subsection{DOE ORDERS}

DOE orders are not promulgated regulations and thus are not considered to be ARARs by EPA. They are, however, legally binding between DOE and Energy Systems because of contractual agreements. The radiation exposure limits defined in DOE Order 5400.5 (Radiation Protection of the Public and the Environment) are: an effective dose equivalent of 100 mrem/year from all exposure pathways and all DOE sources of radiation and a dose of less than $5 \mathrm{rem} / \mathrm{year}$ for a single acute exposure (see Table 9). In addition, effluent releases to surface water must not result in exposures to aquatic organisms exceeding an absorbed dose of $1 \mathrm{rad} / \mathrm{d}$. The overriding principle of the DOE Order is that all releases of radioactive material shall be ALARA. Table 11 summarizes the contents of DOE Order 5400.5 with regard to remedial actions.

DOE Order 5820.2A (Radioactive Waste Management) states that the management of low-level radioactive waste must ensure that external exposure to the waste and concentrations of radioactive material that may be released into surface water and soil does not exceed $25 \mathrm{mrem} / \mathrm{year}$ to any member of the public. Releases to the atmosphere shall not exceed 10 mrem/year (Table 9). Reasonable effort should be made to maintain releases to the environment to ALARA levels. The order pertains to the management of radioactive waste that is not mixed with RCRA hazardous waste; Table 12 summarizes the substantive requirements of this order.

DOE Order 5480.11 (Radiation Protection for Occupational Workers) contains guidelines for worker protection that are similar to those of 10 CFR 20 (i.e., 5 rem/year annual effective dose equivalent from both internal and external sources for continuous exposures). Also included in the order are standards for the general public when entering a controlled area. Exposures to the public are limited to $100 \mathrm{mrem} / \mathrm{year}$. As with the other DOE orders, the ALARA principle prevails. Remediation of radionuclide-contaminated soils must ensure that exposures to on-site workers or public intruders will not exceed these standards. DOE has proposed for codification in 10 CFR 835 the primary standards for radiation protection of occupational workers from normal operation of DOE facilities (56 FR 64334, December 9, 1991). No date for a final rule has been established; however, when promulgated, these standards will then be legally applicable for CERCLA cleanup at DOE sites.

\subsection{TBC GUIDANCE FOR RADIOLOGICAL RISK ASSESSMENT}

The EPA Office of Radiation Programs has derived slope and unit risk factors for radionuclides of concern at remedial sites for each of three major exposure pathways (inhalation, ingestion, and external exposure to contuminated soil). These are available in the EPA Health Effects Assessment Summary Tables (EPA 1991c). 
Table 11. DOE Orier 5400.5 "Radiation Protection of the Public and the Baviranment"

Action
Planning and performance of all
DOE activities

Routine DOE activities including remedial action

Management and storage of spent nuclear fuel, high-level or TRU waste at a disposal facility that is not regulated by the NRC

Disscharge of liquid waste containing radionuclides to surface water
Requirement

Chapter

DOE will carry out all DOE activities to ensure that 1.4 radiation doses to individuals will be as low as reasonably achievable (ALARA).

DOE must comply with all legally applicable requirements of 40 CFR 61 (NESHAPS ${ }^{a}$ for DOE facilities) and 40 CFR 91 (Spent Nuciear Fuel, High-level, and TRU ${ }^{b}$ Wastes).

Exposures to members of the public from all radiation sources shall not cause an effective dose equivalent to be greater than 100 mrem ( $1 \mathrm{mSv}$ ) per year.

Exposures to members of the public from all II.1b radiation sources reledsed into the atmosphere shall not cause an effective sose equivaient to be greater than 10 mrem $(0.1 \mathrm{mSv})$ per year.

Members of the public shall not receive an effective dose equivalent greater than $25 \mathrm{mrem}(0.25 \mathrm{mSv}$ ) to the whole body or $75 \mathrm{mrem}(0.75 \mathrm{mSv})$ to a single organ per year.

Radioactive materials released into public drinking water supplies shall not cause an effective doce equivalent to be greater than $4 \mathrm{mrem}(0.4 \mathrm{mSv})$ io an individual per year. Gross alpha activity shall not exceed $1.5 \times 10^{-8} \mathrm{MCi} i m \mathrm{ml}$.

Liquid effluents shall not cause private or public drinking water systems downstream of the facility to exceed the MCL(s) of $40 \mathrm{CFR} 141$.

Impiementation of best availavie technology (BAT) treatment required if discharge is at an annual average concentration greater than the derived concentration gulde (DCC) level.

Discharge of liquid waste containing radionuclides to surface water at an annual average concentration less than the DCG level does not require the implementation of BAT treatment.

The absorbed doxe to native squatic animal organisms shall not exceed $1 \mathrm{rad} / \mathrm{d}$ from exposurs to liquid wastes discharged to natural waterways.

Liquid wasttes containing concentrations of radioactive materials greater than 5 times the DCG(s) may be discharged to a federal sanitary sewerage system which provides tiquid waste ireatment prior to discharge to surface water according to II.3a(1). ALARA process considerations are required.

If a waste stream contains multiple radionuclides, then the DCG shall be the sum of the fractional $11.3 a(3)$ DCG values. 
Table 11. (continued)

Action
Management of low-level radioactive
solid waste containing no RCRA
constituents
Demonstration of compliance with
the dose limits
Conduct of radiological
environmental protection programs

Cleanup of residual radioactive material and management of resulting wastes or residues
Design, operational, and monitoring requirements are found in DOE Order 5820.2A (Radioactive Waste Management).

DOE shall make appropriate documentation containing information about compliance with dose limits for members of the public in the immediate vicinity and within $80 \mathrm{~km}$ of the site.

DCG(s) for water ingestion, air inhalation, and ir umersion in a gaseous cloud are provided as reference values. These DCG(s) are based on a committed effective dose equivalent of $100 \mathrm{mrem}$ for the radionuclide taken into the body by ingestion or inhalation during one year.

The DCG values apply to only one mode of exposure (i.e., either ingestion or- intralation).

For known mixtures of radionuclides the sum of the ratios of the observed concentration of each radionuclides to its corresponding DCG must not exceed 1.0 .

DOE facilities shall develop plans and protocols for the implementation of this guidance.

These guidelines are for levels of residual radioactive material thai is acceptable for public use of property witbout restrictions because of residual padionctive material.

Generic guidelines are taken from existing radiation protection standards.

Authorized limits are levels of residual radioactive Chapter

II. 4

II.6

III.2

III.2c

IV.1 \& 2

IV.2c material that shall not be excceded if the remedial action is to be considered completed and the property is to be released without restrictions on use.

Monitoring, cleanup, and control of residual radioactive material are subject to the Al.ARA policy.

The basic doxe linits for exposure to residual radioactive material is 100 mrem (1 mSv) annually.

Under unusual circumstances, the dose limit for exposure to residual radioactive material is increased from $100(1 \mathrm{mSv})$ to $500(5 \mathrm{mSv})$ mrem annually.

Residual radioactive material in soil are those concentrations in excess of background concentrations averaged ower an area of $100 \mathrm{~m}^{2}$. These derivations arc obtained by means of snvironmental pathway analysis and basic dose limits. 
Table 11. (continued)

\begin{tabular}{|c|c|c|}
\hline Action & Requirement & Chapter \\
\hline & $\begin{array}{l}\text { Residual concentrations of radionuclides in the air } \\
\text { and water shall not exceed } 100 \text { mrem ( } 1 \mathrm{mSv} \text { ) per } \\
\text { year. }\end{array}$ & IV.4e \\
\hline & $\begin{array}{l}\text { Limits for hot spots shail be developed in cases } \\
\text { where the average concentration in any surface or } \\
\text { below surface area equal to or less than } 25 \mathrm{~m}^{2} \\
\text { exceeds the limit or guidelines by a factor of } \\
(100 / \mathrm{A})^{0.5} \text {. Reusonable effort must be made to } \\
\text { remove any radionuclide source that exceeds } 30 \\
\text { times the limit. }\end{array}$ & IV.4a(1) \\
\hline $\begin{array}{l}\text { Cleanup of residual radioactive } \\
\text { material and management of } \\
\text { resulting wastes or residues } \\
\text { (continued) }\end{array}$ & $\begin{array}{l}\text { The generic guidelines for }{ }^{266} \mathrm{Ra},{ }^{208} \mathrm{Ra},{ }^{230} \mathrm{Th} \text {, and } \\
{ }^{232} \mathrm{Th} \text { are } 5 \mathrm{pCi} / \mathrm{g} \text { averaged over the first } 15 \mathrm{~cm} \text { of } \\
\text { soil or } 15 \mathrm{pCi} / \mathrm{g} \text { averaged over } 15-\mathrm{cm} \text {-thick layers of } \\
\text { soil beiow the first } 15 \mathrm{~cm} \text {. }\end{array}$ & IV. $4 a(2)$ \\
\hline \multirow[t]{8}{*}{$\begin{array}{l}\text { Management of residual radioactivity } \\
\text { above the guidelines specified in } \\
\text { IV.1-4. }\end{array}$} & $\begin{array}{l}\text { Interim storage control and stabilization features } \\
\text { shall be planned to have a minimum life of } 25 \text { years } \\
\text { and if reasonably achievable an effective life of } \\
50 \text { years. }\end{array}$ & IV.6b(1) \\
\hline & $\begin{array}{l}\text { Control features must be designed so that } \\
\text { radionuclide concentration in the groundwater shall } \\
\text { not exceed applicable federal and state standards. }\end{array}$ & IV.6b(3) \\
\hline & $\begin{array}{l}\text { If residual radioactive material is in inaccessible } \\
\text { locations and would be unreasonably costly to } \\
\text { remove and when residual radioactive material } \\
\text { exceeds guideline values, an interim management } \\
\text { plan may be arranged to maintain the property. }\end{array}$ & IV. $6 c(1)$ \\
\hline & $\begin{array}{l}\text { Administrative controls shall include (but are not } \\
\text { limited to): } \\
\text { - periodic monitoring as appropriate; } \\
\text { - appropriate shieiding; } \\
\text { - physical barriers to prevent access; and } \\
\text { - appropriate radiological safety measures. }\end{array}$ & IV.6c(2) \\
\hline & $\begin{array}{l}\text { Control and stabilization for uranium, thorium, and } \\
\text { their decay products must be designed to last at a } \\
\text { minimum of } 200 \text { years and if reasonably achievable } \\
\text { have an effective life of } 1000 \text { years. }\end{array}$ & $\begin{array}{l}\text { IV.6d(1)(a } \\
\end{array}$ \\
\hline & $\begin{array}{l}\text { In accordance with legally applicable federal and } \\
\text { state standards, DOE facilities shall protect } \\
\text { groundwater. }\end{array}$ & $\begin{array}{l}\text { IV.6d(1)(d } \\
\text { ) }\end{array}$ \\
\hline & $\begin{array}{l}\text { Use of and access to residual radioactive material } \\
\text { shall be controlled through appropriate } \\
\text { administrative and physical controls. }\end{array}$ & $\begin{array}{l}\text { IV.6d(1)(e } \\
{ }^{2}\end{array}$ \\
\hline & $\begin{array}{l}\text { Long-term management of other radionuclides shall } \\
\text { be controlled by applicable requiremerits in } \\
\text { Chapters II, III, and IV of DOE } 5820.2 \mathrm{~A}\end{array}$ & IV. $6 \mathrm{~d}(2)$ \\
\hline
\end{tabular}

\footnotetext{
${ }^{a}$ NESHAPS $=$ National Emission Standards for Hazardous Air Pollutants

TTRU = transuranic
} 
Table 12. DOE Order 5820.2A "Radioactive Waste Management"

\begin{tabular}{|c|c|c|}
\hline Action & Requirement & Chapter \\
\hline \multirow[t]{6}{*}{$\begin{array}{l}\text { Management of low-level } \\
\text { radioactive waste }\end{array}$} & $\begin{array}{l}\text { Development of site-specific comprehensive } \\
\text { closure plans shall address: } \\
\text { - Disposal site's closure after the site has } \\
\text { been filled for } 5 \text { years and- } \\
\text { - National Environmental Policy Act (NEPA) } \\
\text { requirements }\end{array}$ & III.3j(1) \\
\hline & $\begin{array}{l}\text { Residual radioactivity levels for surface soil shall } \\
\text { comply with DOE decommissioning guidelines } \\
\text { (DOE Order 5400.5) }\end{array}$ & III.3j(2) \\
\hline & $\begin{array}{l}\text { The Paducah Gaseous Diffusion Plant must } \\
\text { correct problems that could jeopardize the } \\
\text { attainment of this order's performance objectives }\end{array}$ & III. $3 \mathrm{j}(3)$ \\
\hline & $\begin{array}{l}\text { All disposal sites and units shall be maintained } \\
\text { to conform with Resource Conservation and } \\
\text { Recovery Act; Comprehensive Environmental } \\
\text { Response, Compensation, and Liability Act } \\
\text { (CERCLA); and Superfund Amendments and } \\
\text { Reauthorization Act (SARA) standards }\end{array}$ & III. $3 \mathrm{j}(4)$ \\
\hline & $\begin{array}{l}\text { An appropriate field organization shall review } \\
\text { and approve any closure plans for new and } \\
\text { existing low-level waste disposal sites }\end{array}$ & III. $3 \mathrm{j}(5)$ \\
\hline & $\begin{array}{l}\text { An analysis of site performance will be the basis } \\
\text { for terminating the monitoring and maintenance } \\
\text { activity at closed facilities or sites at the end of } \\
\text { the institutional control period }\end{array}$ & III.3j(6) \\
\hline \multirow[t]{3}{*}{$\begin{array}{l}\text { Management of buried } \\
\text { transuranic waste }\end{array}$} & $\begin{array}{l}\text { All disposal sites and units shall be maintained } \\
\text { to conform with CERCLA and SARA standards }\end{array}$ & II. 1 \\
\hline & $\begin{array}{l}\text { Transuranic waste shall be managed to protect } \\
\text { the health and safety of the public and the } \\
\text { workers at the site }\end{array}$ & II. 2 \\
\hline & $\begin{array}{l}\text { Each DOE facility will develop a site closure } \\
\text { plan which will include: } \\
\text { - NEPA requirements } \\
\text { - Applicable federal, state, and local regulations } \\
\text { - Permits required } \\
\text { - Selected closure strategy and justifisation } \\
\text { - Postclosure monitoring and control }\end{array}$ & II. $3 i(4)$ \\
\hline
\end{tabular}




\section{LOCATION-SPECIFIC ARARs}

Location-specific requirements "set restrictions upon the concentration of hazardous substances or the conduct of activities solely because they are in special locations" (53 FR 51394). In determining the use of location-specific ARARs for selection of remedial actions at CERCLA sites, one must investigate the jurisdictional prerequisites of each of the regulations. Basic definitions, exemptions, etc., should be analyzed on a site-specific basis to confirm the correct application of the requirements. Table 13 lists the major federal and state location-specific ARARs that might be pertinent to remedial actions at PGDP. These will be considered further as site-specific remedial alternatives are selected for cleanup at PGDP.

\subsection{CAVES, SALT-DOME FORMATIONS, SALT-BED FORMATIONS, AND UNDERGROUND MINES}

PGDP is located in the Coastal Plain Physiographic Province of western Kentucky. A variety of geologic formations underlie PGDP; the most important formations are the Cretaceous, Paleocene McNairy, and Clayton groups. The bedrock consists of interlaminated and interlensing clay, silt, and sand. The formations are described as micaceous and lignitic, gray-to-dark-gray clay, interlaminated with silt and fine-grained sand. There are no indications of caves, salt-dome formations, salt-bed formations, or underground mines on or near PGDP.

\subsection{FAULTS}

Although reports indicate that there are no faults within the PGDP Reservation, faults lie to the east and west of PGDP (K. R. Davis 1991). These faults near PGDP are ancient (pre-Holocene) and stable. The possibility of fault movement is considered extremely unlikely. In 1811 and 1812, the largest reported earthquake for the area was recorded along the New Madrid fault, 15 miles west of PGDP. However, there has been no reported faulting in Holocene time (K. R. Davis 1991).

PGDP is exempted, however, from compliance with the RCRA seismic requirements of 40 CFR 264.18 because 40 CFR 264.18(a) stipulates that all facilities that are located within political jurisdictions other than those listed in Appendix VI of 40 CFR 264.18 are assumed to be in compliance for location of new TSD facilities. Kentucky is not listed in the Appendix. However, EPA intends to propose additional floodplain and seismic restrictions for location of TSD facilities (NPRM, March 1992; Final Rule expected March 1994), and these restrictions may be ARARs for remedial actions at PGDP.

\subsection{WILDERNESS AREAS, WILDLIFE REFUGES, AND SCENIC RIVERS}

There are no federal wilderness areas, wildlife refuges, or scenic rivers near PGDP. However, the land between the plant boundary and the Ohio River was deeded or leased to the Kentucky Department of Natural Resources and Environmental Protection as part of the West Kentucky Wildlife Management Area (WKWMA). The WKWMA and the surrounding rural farm land provide habitat for many mammalian and avian species. There are no federal 


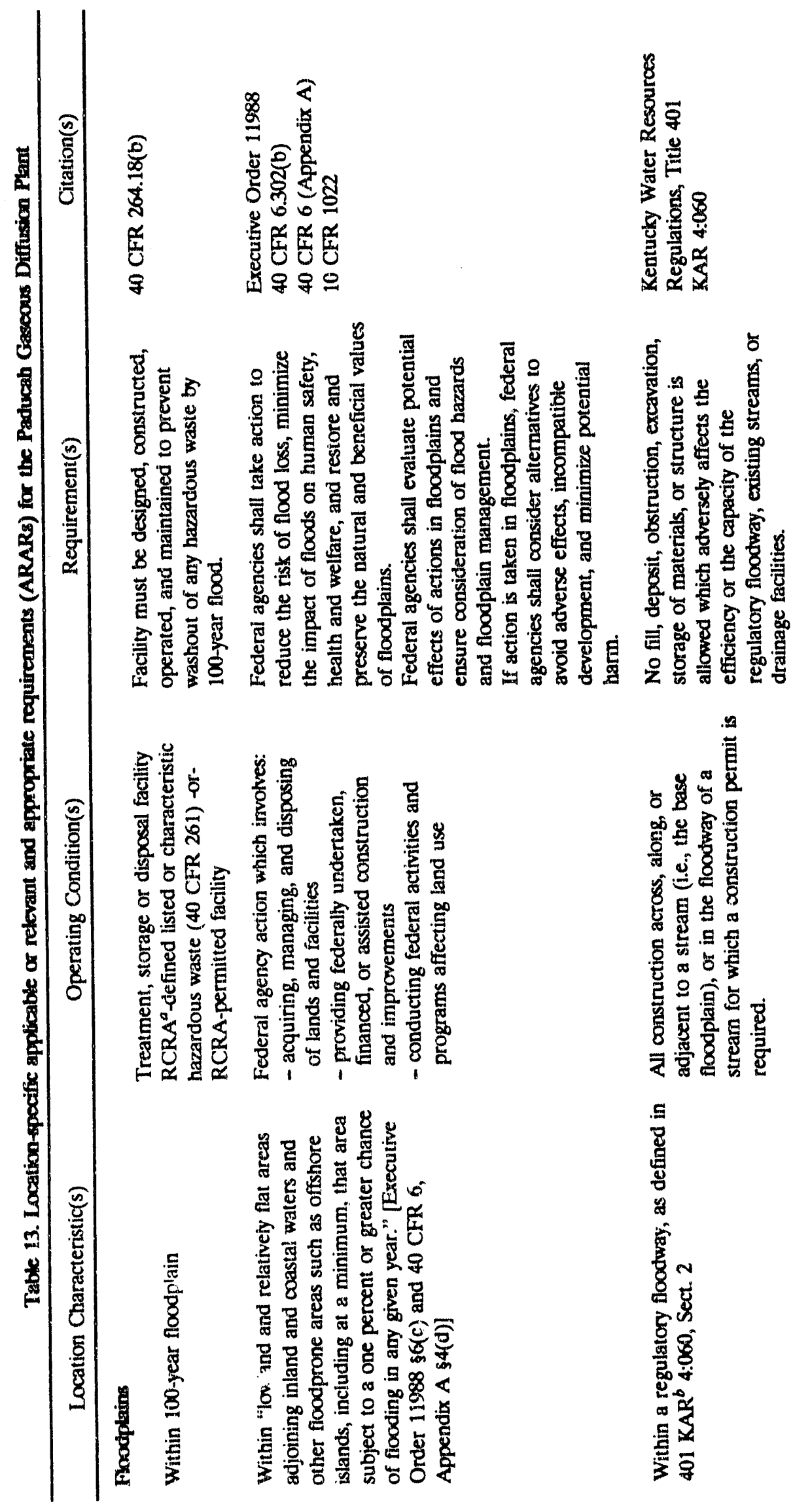




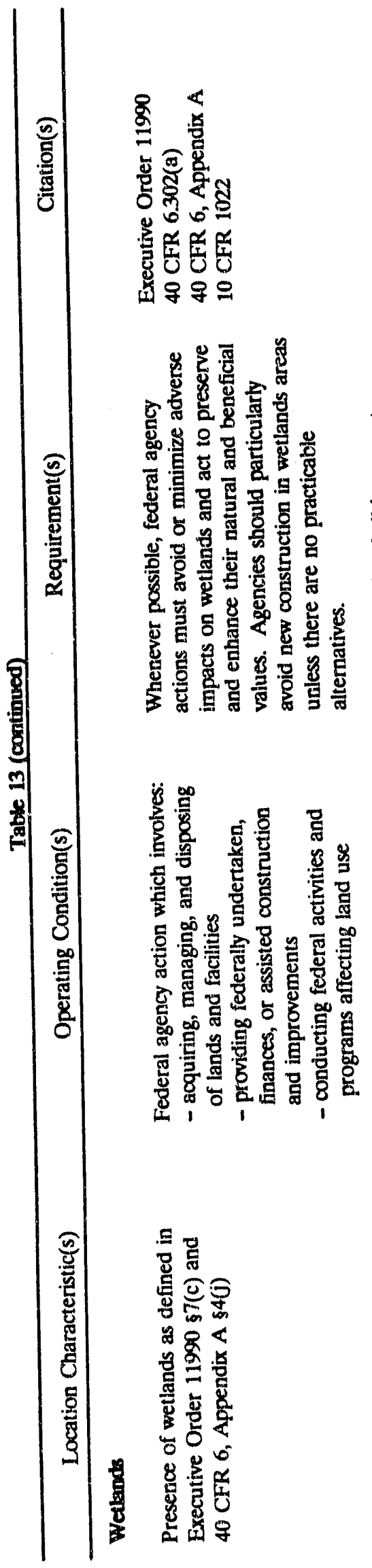

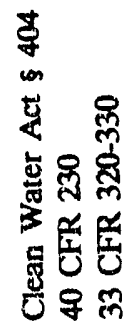
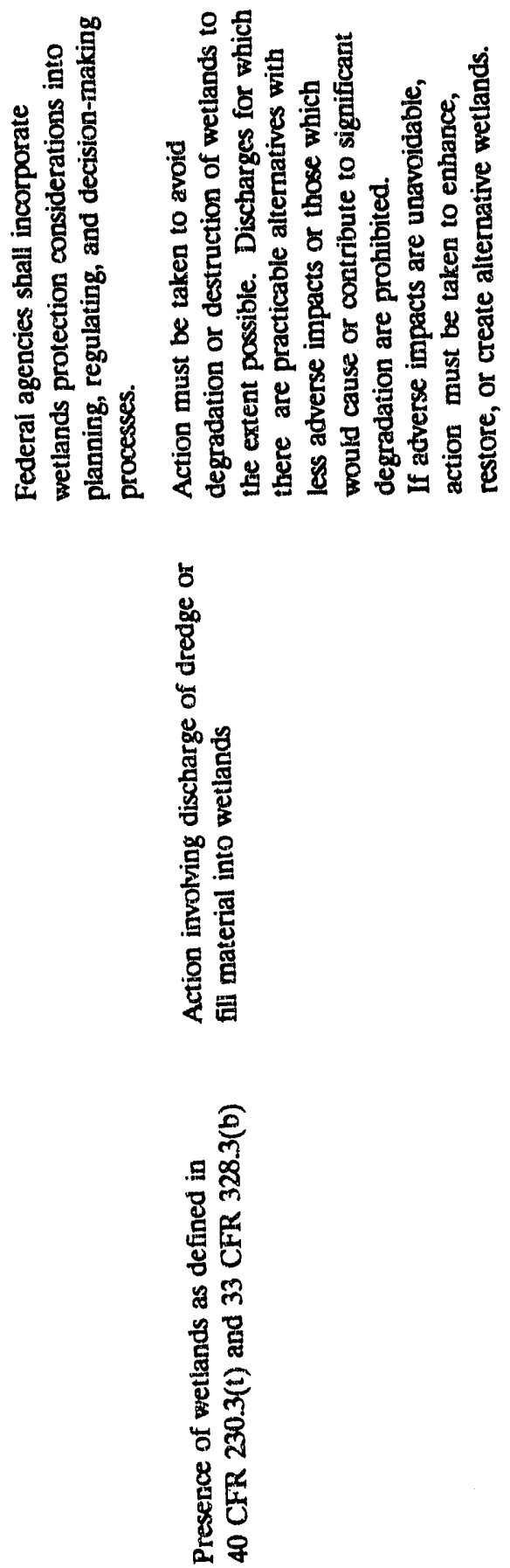


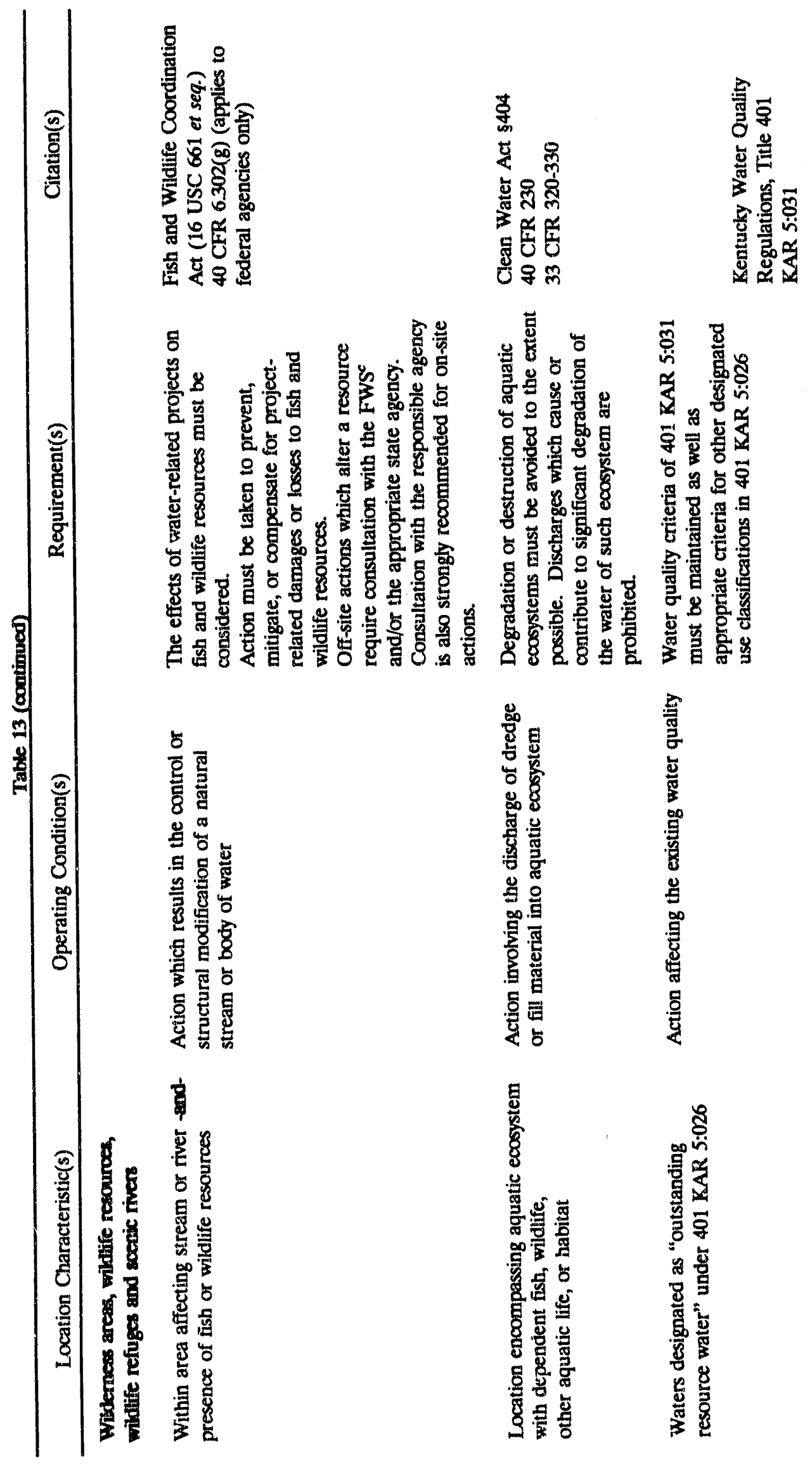




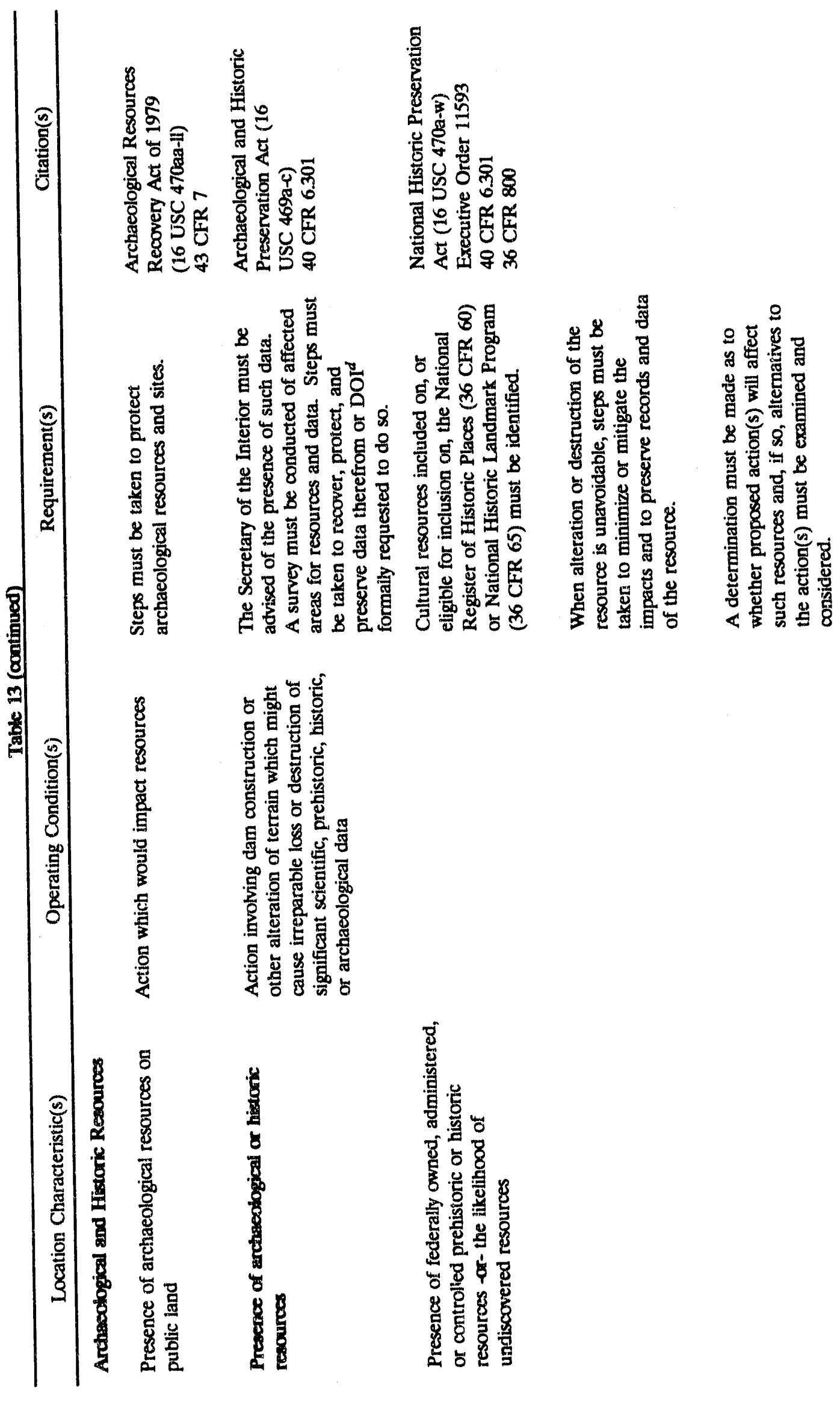



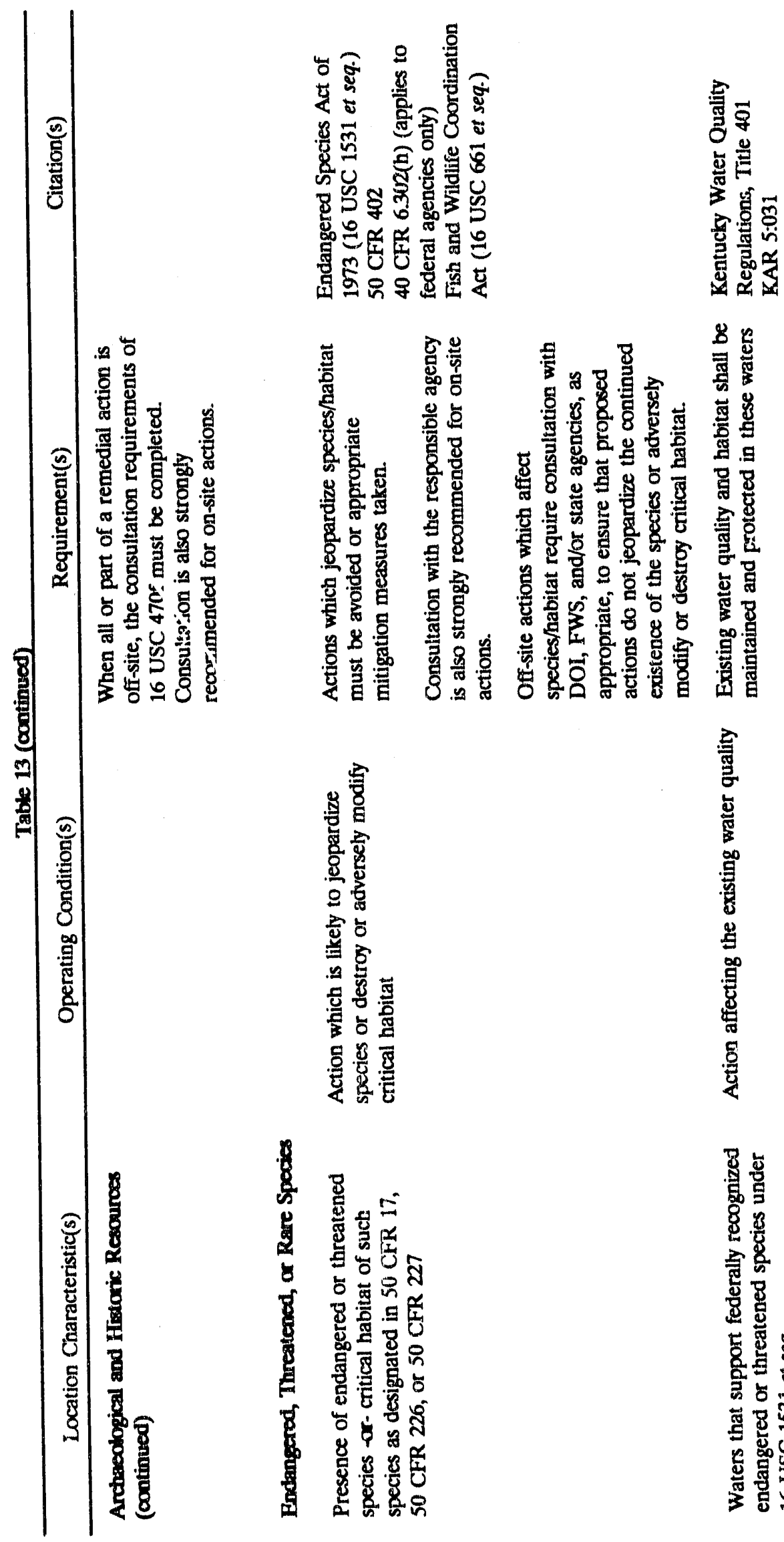

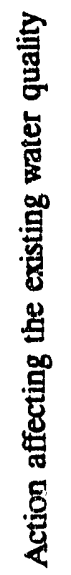
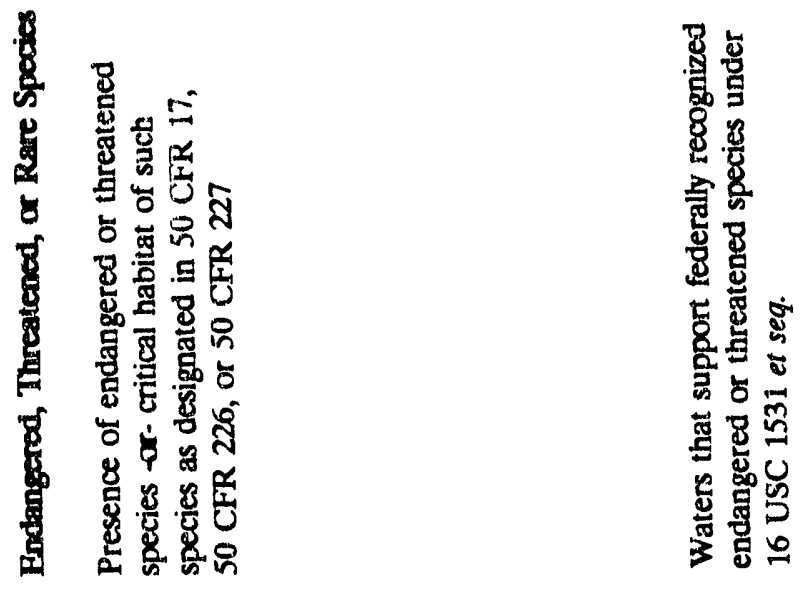


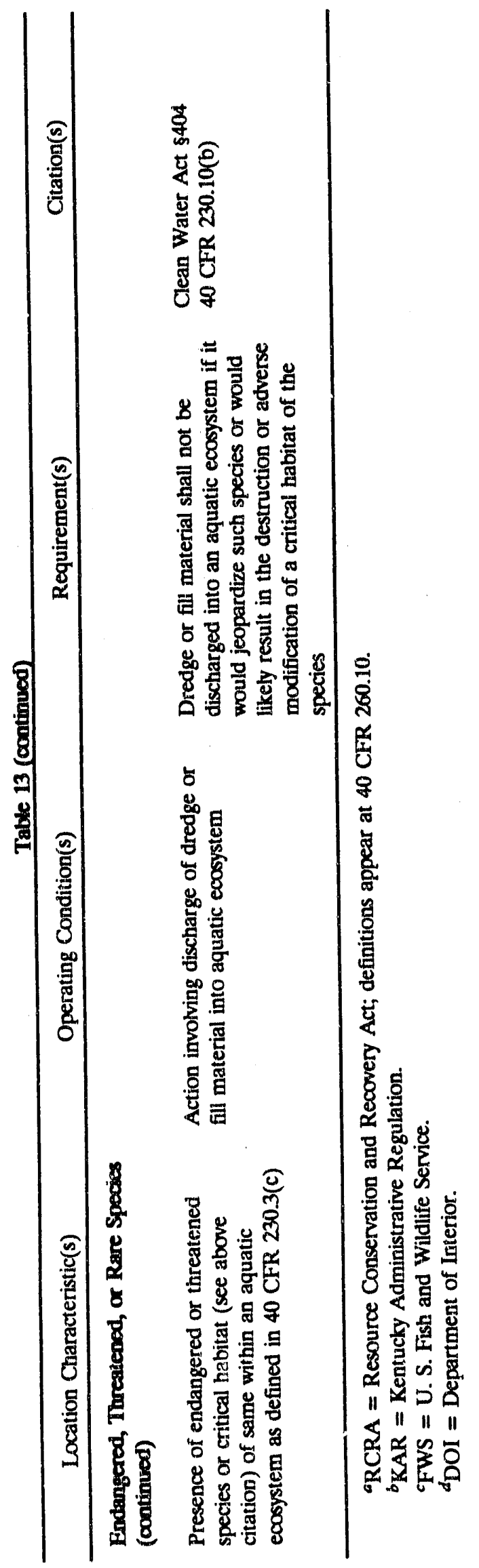


or state regulations specifically applicable to wildlife management areas. However, the Kentucky Department of Fish and Wildlife (KDFW) manages the area. In the event that any remedial activities occurring within the WKWMA would impact the area, consultation with the KDFW would be necessary (W. L. Davis 1991).

Two intermittent streams, Big Bayou Creek and Little Bayou Creek, flow through PGDP and discharge into the Ohio River, which is located 3.6 miles from the PGDP site. Surface runoff, process effluents, and stormwater systems from PGDP flow into Big Bayou Creek and Little Bayou Creek, both of which show chemical and radiological contamination. If any remedial action is taken which would impact the fish and wildlife resources in those creeks, regulations found in the Fish and Wildlife Coordination Act, CWA \$404, 33 CFR 320-330, and 40 CFR 230 may be ARAR (see Table 13).

\subsection{WETLANDS AND FLOODPLAINS}

The United States Fish and Wildlife Service identified several sites on the WKWMA as wetlands areas; however, this classification may change because of the new federal approach to identifying and delineating wetlands (W. L. Davis 1991). One of these areas, a tupolo swamp, has been designated as an area of ecological concern by the Kentucky Nature Preserves Commission (KNPC), in conjunction with the KDFW.

PGDP is not located within a 100-year floodplain; however, portions of the WKWMA are within the 330 - $\mathrm{ft}$ contour of a 100 -year floodplain. There is no information regarding the location of a 500-year floodplain in the area of PGDP (Jones 1991a).

If any remedial alternatives are selected that would impact tloodplains or wetland areas, the requirements found in Executive Order (EO) 11988 and EO 11990, 10 CFR 1022, and $\$ 404$ of CWA might be ARARs for PGDP (Table 13). In addition, 40 CFR 264.18(b) specifies that new TSD facilities must be sited so as to prevent washout from a 100-year flood (Table 13). Title 401 KAR 4:060 provides minimum standards to protect the state's floodprone areas (Table 13); certain activities are excluded from these regulations, providing they do not result in increases of flond elevations (401 KAR 4:060, Sect. 4). These include storage yards, certain sand and gravel operations, and dredging of stream material, providing that disposal of the dredged material is outside the floodplain area. The permitting process of the 401 KAR 6:060 regulations would not be considered ARAR for CERCLA cleanup at PGDP.

\subsection{HISTORIC SITES AND ARCHEOLOGICAL FINDINGS}

Some historic sites and/or archeological findings have been identified near the PGDP site (Jones 1991a). Between the WKWMA and the Ohio River, 21 archeological sites and several archeological findings have been recorded (Schenian 1991). There are also buildings at the adjacent Kentucky Ordnance Works and several old homesteads that could be placed on the National Register. In the WKWMA, several cemeteries and areas containing cultural artifacts have been identified (Jones 1991a). In addition, Building 340, within the security fence, may qualify for the National Register of Historic Places. A cultural resources study may be initiated by the PGDP NEPA Office and the building submitted as a candidate for listing (Jones 1991b). 
If any remedial action is taken that would cause irreparable harm, loss, or destruction of any prehistoric or historic archeological sites, regulations found in the Archaeological Resources Recovery Act, the Archaeological and Historic Preservation Act, and in the National Historic Preservation Act may be ARARs (See Table 13). There are no state regulations protecting historic or archeological sites.

\subsection{RARE, THREATENED, OR ENDANGERED SPECIES}

The Indiana bat (Myotis sodalis), a federally listed endangered species, has been reported in the WKWMA (Hendricks 1991). Although Indiana bats winter in caves, their breeding areas include mature stands of trees near water, especially rivers and wetlands (McCracken 1991).

There is no Kentucky Endangered Species Act or official state list of endangered and threatened species. However, the Kentucky Academy of Science (KAS) in conjunction with the KNPC has published a list of Kentucky animals and plants that are rare, threatened, endangered, or considered to be of "special concern" at the federal or state level as of 1986 (KAS-KNPC 1986). In addition, the KDFW maintains a database of threatened or endangered species listed by county and U. S. Geological Survey quadrant.

A recent survey at the WKWMA indicated the presence of state-listed threatened or endangered bats, birds, amphibians, and plants (see Table 14). There are also several species listed as of special concern by the state which have been collected in the WKWMA (Hendricks 1991). The results of this survey are found in a KNPC database. Metropolis Lake also contains several state-listed animals (see Table 14). Thu re is fairly good agreement between the McCracken County/Joppa Quadrangle list supplied by the KDFW and the KNPC database. However, three species listed by the KDFW as occurring in McCracken County have not been reported in the Nature Preserves Commission database. These are the lake chubsucker (Erimyzon sucetta), listed as state threatened, and the black buffalo (Ictiobus niger) and northern madtom (Noturus stigmosus), both listed as of "special concern" for the state. The black buffalo is found in large rivers and the northern madtom occurs in large creeks to moderate rivers; the lake chubsucker may be found in swampy habitat or oxbow lakes (Etnier 1991). None of these three species were found to occur in Little or Big Bayou Creeks during a recent stream survey (Ryon 1991), although the lake chubsucker was reported as rare in these creeks in an earlier survey.

The Kentucky Water Quality regulations designate streams, rivers, and surface waters that support endangered or threatened species as defined under the Endangered Species Act of 1973, as amended (16 U.S.C. 1531 et seq.), as "outstanding resource waters" (ORW). The presence of federally endangered or threatened species, or waters within a formally dedicated nature preserve, are used in designating ORWs (401 KAR 5:031, Sect. 7, he Ohio River, from river mile 940.7 to river mile 943.3 and river mile 966.3 to river mile 969.5 in McCracken and Ballard counties has been designated as ORW (401 KAR 5:026) because of the presence of a federally listed endangered clam (Lampsilis orbiculata), and as such is protected (401 KAR 5:031, Sect. 7). Metropolis Lake presents a unique floodplain habitat which is ecologically interesting and so has also been designated ORW (Schneider 1991). 
If a site investigation reveals the presence of breeding colonies of Indiana bats or any other federally listed threatened or endangered animals or plants in the vicinity of PGDP, the federal Endangered Species Act of 1973 may be ARAR (Table 13).

Tuble 14. Federally tisted and state-fisted threatenod and endangered spocies found at West Kentucky Widlife Management Area and Metropolis Iakce

\begin{tabular}{|c|c|c|}
\hline $\begin{array}{c}\text { Species name } \\
\text { (common name) }\end{array}$ & Federal listing ${ }^{b}$ & State listing ${ }^{b}$ \\
\hline \multicolumn{3}{|c|}{ West Kentucky Wildlife Mannagement Area } \\
\hline $\begin{array}{l}\text { Silphium lacinietum } \\
\text { (Compass plant) }\end{array}$ & - & $\mathbf{s}$ \\
\hline $\begin{array}{l}\text { Hyla cinerea } \\
\text { (Green tree frog) }\end{array}$ & - & $\mathrm{T}$ \\
\hline $\begin{array}{l}\text { Rana areolata } \\
\text { (Crawfish frog) }\end{array}$ & - & $\mathbf{S}$ \\
\hline $\begin{array}{l}\text { Lophodytes cucullatus } \\
\text { (Hooded merganser) }\end{array}$ & - & $\mathrm{E}$ \\
\hline $\begin{array}{l}\text { Vireo belli } \\
\text { (Bell's vireo) }\end{array}$ & - & s \\
\hline $\begin{array}{l}\text { Nycticeius hummeralis } \\
\text { (Evening bat) }\end{array}$ & - & $\mathrm{T}$ \\
\hline $\begin{array}{l}\text { Myotis septentrionalis } \\
\text { (Keen's Myotis) }\end{array}$ & - & $\mathbf{S}$ \\
\hline $\begin{array}{l}\text { Myotis sodalis } \\
\text { (Indiana bat) }\end{array}$ & $\mathrm{E}$ & $\mathrm{E}$ \\
\hline \multicolumn{3}{|c|}{ Medropclis Lake } \\
\hline $\begin{array}{l}\text { Orconectes lucifer } \\
\text { (Crayfish) }\end{array}$ & - & $\mathbf{E}$ \\
\hline $\begin{array}{l}\text { Esox niger } \\
\text { (Chain pickerel) }\end{array}$ & - & $\mathbf{S}$ \\
\hline $\begin{array}{l}\text { Hybognathus hayi } \\
\text { (Cypress minnow) }\end{array}$ & - & $\mathbf{E}$ \\
\hline $\begin{array}{l}\text { Notropis maculatus } \\
\text { (Tailgate shiner) }\end{array}$ & - & $\mathbf{T}$ \\
\hline $\begin{array}{l}\text { Lepomis punctatus } \\
\text { (Spotted sunfish) }\end{array}$ & - & $\mathrm{T}$ \\
\hline
\end{tabular}

${ }^{a}$ Kentucky Nature Preserves Commission database, September 1991 (Hendricks 1991).

${ }^{b} \mathrm{~S}=$ species of special concern; $\mathrm{T}=$ threatened species; $\mathrm{E}=$ endangered species; $\mathrm{C} 2=$ species under consideration for federal listing but for which insufficient data exist to support a listing. 


\section{ACTION-SPECIFIC ARARs}

Performance, design, or other action-specific requirements set controls or restrictions on particular kinds of activities related to the management of hazardous waste (52 FR 32496). Selection of a particular remedial action at a site will invoke the appropriate action-specific ARARs that may specify particular performance standards or technologies, as well as specific environmental levels for discharged or residual chemicals. Action-specific ARARs are established under RCRA, CAA, CWA, SDWA, and TSCA. These regulations are briefly summarized here and will be expanded as site-specific ARARs are developed.

Several Kentucky statutes also apply to remedial actions at PGDP. These include the air quality regulations found at $401 \mathrm{KAR}$, Chapters 50-65; the underground storage tank (UST) regulations at $401 \mathrm{KAR}$ 42:60 (corrective action) and 401 KAR 42:70 (closure); and the waste management regulations at $401 \mathrm{KAR}$ Chapters 30-49.

\subsection{RESOURCE CONSERVATION AND RECOVERY ACT}

RCRA has nine distinct sections (subtitles) that regulate various aspects of hazardous waste. Three of these, Subtitle C "Hazardous Waste Managennent," Subtitle D "Solid Waste Management," and Subtitle I "Regulation of Underground Storage Tanks," proride the regulatory guidance for RCRA and will be considered for ARAR selection for on "site cleanup at PGDP. In considering compliance of CERCLA remedial actions with RCRA requirements, the Subtitle $\mathrm{C}$ and Subtitle I regulations will most likely be applicable or relevant and appropriate. Although the Subtille $D$ regulations will not be legally applicable, they may prove relevant and appropriate to remediation of sites at PGDP.

\subsubsection{RCRA Subtitle C Regulations}

RCRA Subtitle $C$ regulates the generation, transportation, treatment, storage, and disposal of hazardous wasie. Standards for the management of hazardous waste at RCRApernitted TSD facilities were promulgated July 26, 1932, and appear as 40 CFR 264. Stanciards for interim TSD facilities are found in 40 CFR 265.

The Subtitle C Part 264 standards will be considered first as ARARs, and the interim standards will be considered only if Part 264 does not address a particular issue. In general, this text will address ine Part 264 rather than the Part 265 standards as they are the more stringent. Table 15 lists the pertinent subparts of the federal RCRA permitted and interim standards and the equivalent section of the Kentucky hazardous waste management regulations.

The administrative requirements of RCRA (e.g., permitting, reporting, and recordkeeping) are not required for on-site CERCLA remedial actions [CERCLA $\$ 121(\mathrm{e})$ ]. However, under CERCLA $\$ 121$ (d)(3), any removal or remedial action involving the off-site transfer of hazardous substances, pollutants, or contaminants must involve a facility in compliance with all RCRA substantive and administrative requirements. In the event that PGDP is placed on the NPL, it is assumed that CERCLA response actions will be coordinated with and supplement the corrective measures required and conducted by DOE under its current $\overline{\mathrm{K}} \mathrm{CK} \dot{\mathrm{s}}$ permit. 
The RCRA Part 264 regulations apply to particular waste management processes (eg., capping or incineration) or to general procedures (eg., treatment, disposal, or closure), and it may be necessary to combine the two types of categories to adequately address the actionspecific ARARs for a selected remedial action. These issues will be addressed on a sitespecific basis following selection of remedial alternatives.

Table 15. RCRA“ Subtitle C - standands for the treatment, storage, and disposal of hazardous waste

\begin{tabular}{lll}
\hline \multicolumn{1}{c}{ Federal } & \multicolumn{1}{c}{ Kentucky } & \multicolumn{1}{c}{ Cattegory } \\
\hline 40 CFR Part 264 & 401 KAR $^{\dagger} 34$ & \\
Subpart I & Sect. 180 & Containers \\
Subpart J & Sect. 190 & Tanks \\
Subpart K & Sect. 200 & Surface inpoundments \\
Subpart L & Sect. 210 & Waste piles \\
Subpart M & Sect. 220 & Land treatment \\
Subpart N & Sect. 230 & Landfills \\
Subpart O & Sect. 240 & Incinerators \\
Subpart X & Sect. 250 & Miscellaneous treatment units \\
40 CFR Part 265 & 401 KAR 35 & \\
Subparts I.O & Sects. $180-240$ & As listed above \\
Subpart P & Sect. 250 & Thermal treatment \\
Subpart $Q$ & Sect. 260 & Chemical, physical, and \\
Subpart R & Sect. 270 & biological treatment \\
\hline
\end{tabular}

${ }^{a} \mathrm{RCRA}=$ Resource Conservation and Recovery Act

${ }^{b} \mathrm{KAR}=$ Kentucky Administrative Code

\subsubsection{Land Disposal Restrictions}

For each hazardous waste, EPA has established treatment standards that are protective of human health and the environment when the wastes are land disposed. Land disposal includes placement in a landfill, surface impoundment, waste pile, land treatment facility, etc. In the final NCP, EPA reaffirms that movernent of waste within a unit does not constitute "land disposal" for purposes of applicatins of the RCRA LDR; however, waste consolidation from different units at a CERCLA site are subject to the restrictions (55 FR 8759).

These LDR apply only to RCRA hazardous waste placed after the effective prohibition date. Land diposal is acceptable for wastes that have been treated with the best demonstrated 
available technology (BDAT) set by EPA and meet the treatment standards. A final rule listing provisions for specific treatment standards and prohibiting land disposal has appeared. Table 16 lists the waste categories for which LDR have been promulgated, the Federal Register citation, and the corresponding section in 40 CFR 268. Title 401 KAR, Chapter 37, contains the Kentucky Waste Management Land Disposal Restrictions. These are similar to the RCRA LDR, and will be analyzed for site-specific disposal options, if necessary.

Table 16. Federal RCRA* land disposal regulations

\begin{tabular}{|c|c|c|}
\hline Waste category & Citation & 40 CFR Part \\
\hline $\begin{array}{l}\text { Spent-solvent and dioxin- } \\
\text { containing wastes }\end{array}$ & $\begin{array}{l}51 \text { FR } 40572 \\
\text { (November } 7,1986 \text { ) }\end{array}$ & $\begin{array}{l}268.30 \\
268.31\end{array}$ \\
\hline "California Lisı" wastes & $\begin{array}{l}\text { 52. FR } 25760 \\
\text { (July } 8,1987 \text { ) }\end{array}$ & 268.32 \\
\hline First third wastes ${ }^{b}$ & $\begin{array}{l}54 \text { FR } 31138 \\
\text { (August 17, 1988) }\end{array}$ & 268.33 \\
\hline Second third wastes ${ }^{b}$ & $\begin{array}{l}54 F R 26594 \\
\text { (June 23, 1989) }\end{array}$ & 268.34 \\
\hline Final third wastes ${ }^{b}$ & $\begin{array}{l}55 F R 22520 \\
\text { (June } 1,1990 \text { ) }\end{array}$ & 268.35 \\
\hline
\end{tabular}

${ }^{a}$ RCRA $=$ Resource Conservation and Recovery Act.

"All listed hazardous wastes identified under 40 CFR 261.

EPA has determined that the RCRA treatment standards are generally inappropriate or infeasible when applied to contaminated soil or debris (55 FR 8760). Therefore, EPA is proposing separate rulemakings to establish treatment standards for disposal of such contaminated soil and debris. The Advance Notice of Proposed Rulemaking (ANPRM) for debris appeared in 56 FR 24444, May 30, 1991; the NPRM January 9, 1992 (57 FR 958); final rule, June 1992. The proposed rule requires contaminated debris to be treated prior to land disposal using extraction, destruction, or immobilization technologies. Six different categories of debris and ten different categories of contaminants are specified in the rule. As with mixed waste, debris contaminated with both hazardous and radioactive waste must comply with the treatability standards for contaminated debris as well as those under the AEA. Disposal of treated debris and resultant wastes are discussed in the proposed rule.

The ANPRM for soil appeared October 24, 1991 (56 FR 55160); the NPRM is scheduled for May 1992; final rulemaking May 1993. These will be analyzed as ARARs or TBC when available. In the interim, EPA has devcloped guidance for obtaining and complying with a treatability variance for soil and debris that are contaminated with RCRA hazardous wastes for which treatment standards have already been set (OSWER Directive 9347.3-06FS, July 1989). Alternate treatment levels are presented for structural functional groups of organics and for ten inorganics based on actual treatment of soil and best management practices for 
debris. These will be considered as $\mathrm{TBC}$ guidance when remedial alternatives are selected and more information becomes available on waste types.

\subsubsection{Corrective Action Requirements}

Several sections of RCRA require promulgation of corrective action regulations; when finalized, these requirements will most likely be ARARs for CERCLA remedial actions. Currently, only the 40 CFR 264 Subpart F regulations on groundwater protection at units regulated by RCRA are potential ARARs to CERCLA corrective actions within facility boundaries, and these include groundwater protection standards [40 CFR 264.92 and 264.94 (see Sect. 2.1.1)] and groundwater monitoring requirements (40 CFR 264.97). The corrective action standards developed under RCRA $\$ 3004$ (u) will be applicable to any CERCLA cleanup site that is also an active or a formerly active RCRA facility with an active SWMU; thus, the standards will also be applicable to a CERCLA site where disposal is currently occurring. In addition, RCRA $\$ 3004(v)$ authorizes cleanup beyond facility boundaries.

Requirements for corrective action for SWMUs have been proposed (55 FR 30798, July 27, 1990). A final rule is expected to appear in January 1993. EPA proposes a risk-based approach to establish media cleanup standards for surface water, groundwater, soil, and air. Thes: standards are to be established at concentrations that ensure protection of human health and the environment and are to be set for each media of concern during the remedy selection process. Target cleanup levels may initially be set at the RCRA action levels, but modified as appropriate to reflect site-specific exposure assumptions (55 FR 30826).

\subsubsection{RCRA Subtitle I Regulations}

RCRA $\$ 9003$ requires promulgation of regulations pertaining to detection, prevention, and correction of releases from USTs; implementing regulations appear in 40 CFR 280.60, "Release Response and Corrective Action for UST Systems Containing Petroleum or Hazardous Substances," and 40 CFR 280.70, "Out-of-Service UST Systems and Closure," and will be considered as ARARs for cleanup of USTs at PGDP. The corresponding state UST regulations are found at $401 \mathrm{KAR} 42: 060$ and 42:070, respectively, and cite the 40 CFR 280 regulations as governing requirements. 40 CFR 280.66 specifies that corrective action plans designed to protect human health and the environment must be submitted to the state "implementing agency" (UST Program, Division of Waste Management, Kentucky Department for Environmental Protection) for approval. The state must be notified of permanent closure plans not taken in response to corrective action (40 CFR 280.71); suggested cleaning and closure procedures are recommended in 40 CFR 280.71 and may be considered TBC guidance for closure of tanks at PGDP.

\subsubsection{RCRA Subtitle D Regulations}

EPA has promulgated a final rule establishing a new 40 CFR Part 258, which sets forth revised minimum federal criteria for municipal solid waste landfills ( 56 FR 50978, October 9 , 1991). 'These include closure and postclosure care requirements (40 CFR 258.60 and 258.61). Although not legally applicable to remedial action sites found at PGDP, they may prove relevant and appropriate for closure of some of the sites. These regulations will be analyzed as site-specific ARARs are addressed. 
In addition, the Kentucky state and federal RCRA regulations governing solid waste management facilities may be relevant and appropriate. Specifically, 40 CFR 257.3-3 prohibits non-point-source contamination of surface waters from solid waste management facilities. 40 CFR 257.3-3 also prohibits point-source discharges of pollutants into surface waters which are in violation of the requirements of the NPDES (401 KAR 37.030 Sect. 4). 40 CFR 257.34 prohibits contamination of an underground drinking water source above SDWA MCLs (401 KAR 37.030 Sect. 5).

\subsubsection{Federal vs State RCRA Regulations}

Table 17 gives a preliminary list of action-specific ARARs established under RCRA and various other statutes discussed below. Action alternatives are listed alphaberically, followed by a summary of the requirements found in the particular statute cited. The prerequisites for applicability are also given; however, the regulations must be related to site-specific conditions to determine whether the regulation cited is legally applicable or relevant and appropriate. This table does not represent a complete listing of action-specific ARARs; analysis of the regulations on a site-specific basis will be necessary to ensure a comprehensive summary.

Kentucky received final authorization of its hazardous waste program on January 31, 1985 (50 FR 2550), and has since received authorization for several revisions to its program (53 FR 41164, October 20, 1988; 54 FR 1940, January 18, 1989; 54 FR 10986, March 16, 1989). The Kentucky Department for Environmentai Protection, Division of Waste Management, is responsible for regulating hazardous waste. The standards for owners and operators of TSD facilities are found at 401 KAR Chapters 30-49. The Kentucky hazardous waste management regulations closely resemble the federal RCRA regulations (Government Institutes, Inc. 1991), and are listed in Table 17 with the appropriate federal RCRA citation.

\subsection{CLEAN AIR ACT}

The primary ambient air quality standards were established by the Kentucky Division of Air Quality to define levels of air quality which are necessary to protect human health. The implementing regulations are found in 401 KAR 53:010 and are listed in Appendix A of that statute. However, as mentioned previously (Sect. 2.2), these air quality standards apply only to major sources and are unlikely to be ARAR for CERCLA cleanup actions. Similarly, the state regulations governing emissions from existing sources (401 KAR 61 and 401 KAR 63:021) will not generally be considered as ARARs for CERCLA cleanup.

The Kentucky Air Quality regulations contain General Standards of Performance governing fugitive emissions (401 KAR 63:010). Fugitive emissions are emissions of any air contaminant into the open air other than from a stack or air pollution control device. Reasonable precaution must be taken to prevent particulate matter from becoming airborne during handling, processing, construction, road grading, land clearing, etc. Site remedial activities that generate dust will be subject to the provisions of this rfigulation.

The CAA (\$111) requires EPA to promulgate standards for new sources of air emissions. This requirement has been implemented in 40 CFR 60 through the New Source Performance Standards (NSPS) that are based on the best demonstrated technology (BDT) and set minimum federal emission limitations on classes of facilities. The NSPS are probabiy not legally applicable to cleanup of CERCLA sites, but will be considered as potentially relevant 
and appropriate for cleanup at PGDP on a site-specific basis. NESHAPs have been discussed as potential "hybrid" ARARs (see Sects. 2.2.1 and 3.1), which will be considered as remedial alternatives are selected.

\subsection{CLEAN WATER ACT}

The regulatory aspects of the CWA include site-specific pollutant limitations and performance standards designed to restore and maintain the chemical, physical, and biological integrity of the nation's surface waters. The National Pollutant Discharge Elimination System (NPDES) permit program includes applicable effluent standards, monitoring requirements, and conditions for discharge. Kentucky has an EPA-authorized state program that is equivalent to the federal program (401 KAR 5:050-080). Although NPDES permits are not necessary for on-site discharges from CERCLA sites, the substantive CWA NPDES requirements must be complied with. KPDES permit No. KY0004049 regulates discharges from PGDP via 16 outfalls into Big and Little Bayou creeks. The effluent limitations established in this permit will be considered ARARs for discharges resulting from remedial actions at PGDP.

Direct discharge of dredge-and-fill material into navigable waters is regulated under CWA $\$ 404$ and implemented through 33 CFR 320-330 and 40 CFR 230. CERCLA remedial actions that may be considered dredge-and-fill activities include dredging sediments from contaminated water bodies, disposing of contaminated soil or materials in surface water, capping a site, building berms and levees to contain wastes, excavating for the containment of effluent, and dewatering a site to obtain adequate flow (EPA 1988).

The Corps of Engineers issues dredge-and-fill permits for activities regulated under $\$ 10$ of the Rivers and Harbors Act and \$404 of the CWA. CERCLA \$121(e) stipulates that permits are not required for on-site CERCLA cleanup; however, the applicability of these regulations for off-site remediation will be addressed on a site-specific basis.

\subsection{SAFE DRINKING WATER ACT}

Part C of Title XIV of the SDWA authorizes the establishment of an underground injection control (UIC) permit program designed to prevent contamination of underground sources of drinking water. An underground drinking water source is defined in the UIC regulations (40 CFR 146.3) as an aquifer or its portion that supplies drinking water for human consumption, contains a sufficient supply of groundwater to supply a public water system, and contains fewer than $10,000 \mathrm{mg}$ total dissolved solids per liter of water. Although permits are not necessary for CERCLA cleanup [CERCLA \$121(e)], technical criteria and standards applicable to the various classes of underground injection wells (defined in 40 CFR 146.5) appear in 40 CFR 146, and may be ARARs for CERCLA cleanup at PGDP. Kentucky does not have a UIC Program in place; therefore, the SDWA UIC regulations are implemented through the EPA Region IV UIC Section. The UIC standards will be analyzed on a sitespecific basis if underground injection is a remedial option. 


\subsection{TOXIC SUBSTANCES CONTROL ACT}

Specific TSCA standards exist for incineration or alternate disposal of liquid and nonliquid waste and for articles and soils containing PCBs, and these will be addressed during the site-specific RI/FS process. TSCA storage requirements for PCBs specify that PCBs and PCB-containing items at concentrations of greater than $50 \mathrm{ppm}$ must be disposed of within 1 year after being placed in storage (40 CFR 761.65). This presents a problem for radionuclide-contaminated $\mathrm{PCB}$ wasies that may be generated during cleanup at PGDP because of the lack of treatment and mixed-waste disposal capabilities. However, DOE and EPA are currently negotiating the unauthorized storage of these mixed-PCB wastes.

There are also structural requirements for facilities and containers used for storage. These requirements will be addressed on a site-specific basis for those PGDP sites requiring remediation of PCBs or PCB-contaminated materials or soil.

\subsection{FEDERAL INSECTICIDE, FUNGICIDE, AND RODENTICIDE ACT}

The Federal Insecticide, Fungicide, and Rodenticide Act delegates authority to EPA to regulate the disposal and storage of excess pesticides and pesticide containers. To that end, EPA has published nonregulatory procedures for disposal of pesticides in 40 CFR 165, Subpart C. Although not an ARAR, these recommendations might provide guidance for cleanup of pesticides and pesticide containers at PGDP and will be analyzed on a site-specific basis, if necessary. EPA is currently updating these guidelines to make them consistent with current regulations and technologies. Regulatory initiatives for 40 CFR 165 will be published in three phases. An NPRM for Phase I, concerning suspended and cancelled pesticides, will be published in early 1992. The NPRM for Phase II, concerning container design, residue removal storage, containment, and excess, is due in 1993, and the Phase III NPRM is due in 1994.

\subsection{OCCUPATIONAL SAFETY AND HEALTH ADMINISTRATION}

Title I, \$126(b) of CERCLA mandated that OSHA promulgate standards for regulation of employee health and safety during hazardous waste operations at RCRA or CERCLA sites and during emergency response to hazardous substance releases. The final regulations for "Hazardous Waste Operations and Emergency Response" (29 CFR 1910) have appeared in 54 FR 9294 (Final Rule, March 6, 1989; effective date, March 1990). These regulations are designed to protect workers involved in cleanup operations at uncontrolled hazardous waste sites and to provide for worker protection during initial site characterization and analysis, monitoring activities, materials handling activities, training, and emergency response. These regulations do not apply to those workers who would not be exposed. Federal construction activities involving no potential for hazardous substance exposure are covered by the OSHA standards in 29 CFR 1926, "Federal Service Contracts," and 29 CFR 1910, "General Industry."

In general, however, the OSHA standards are not considered to be ARARs for CERCLA remedial action, and will not be addressed unless they contain provisions pertinent to protection of the environment. 


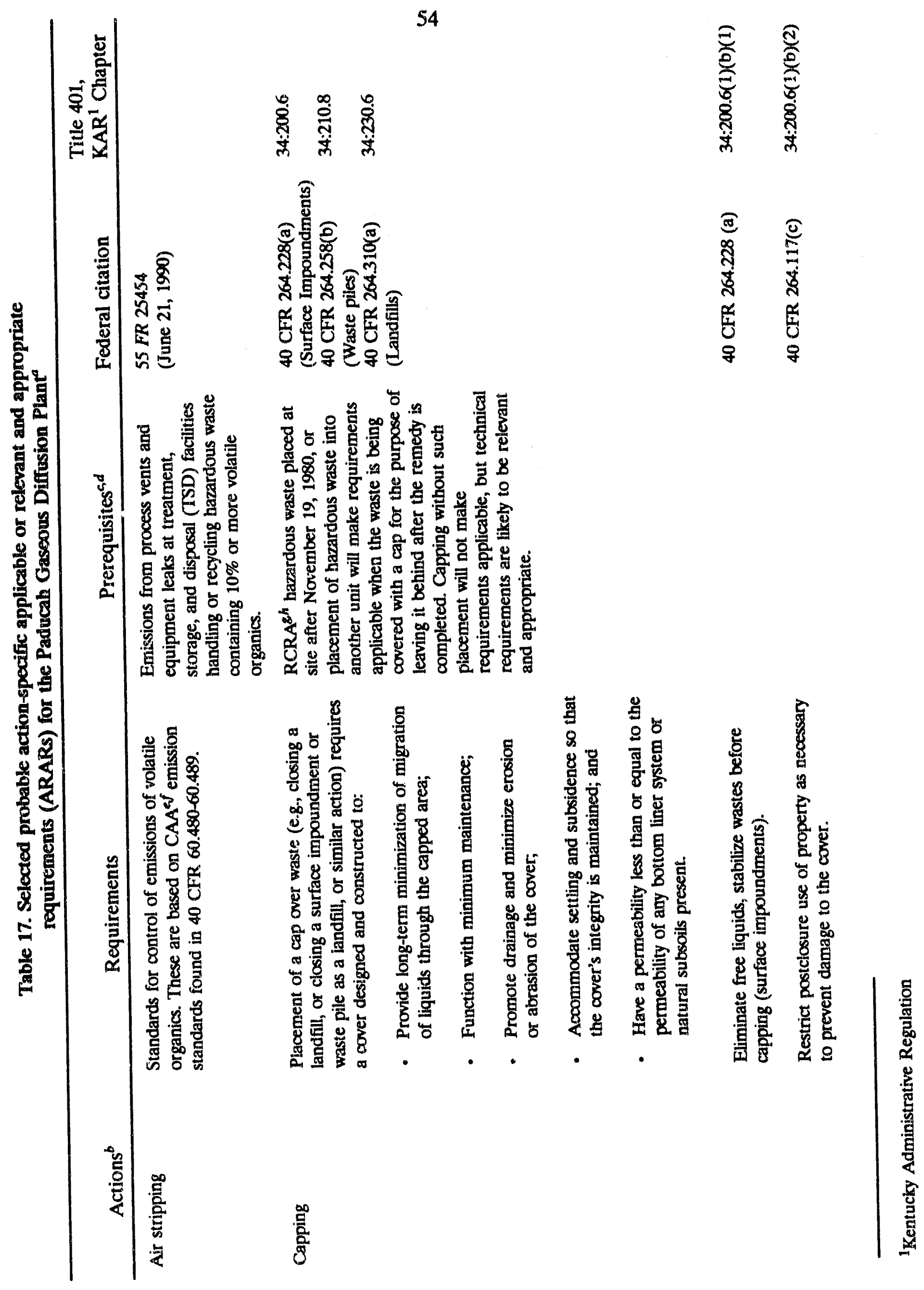




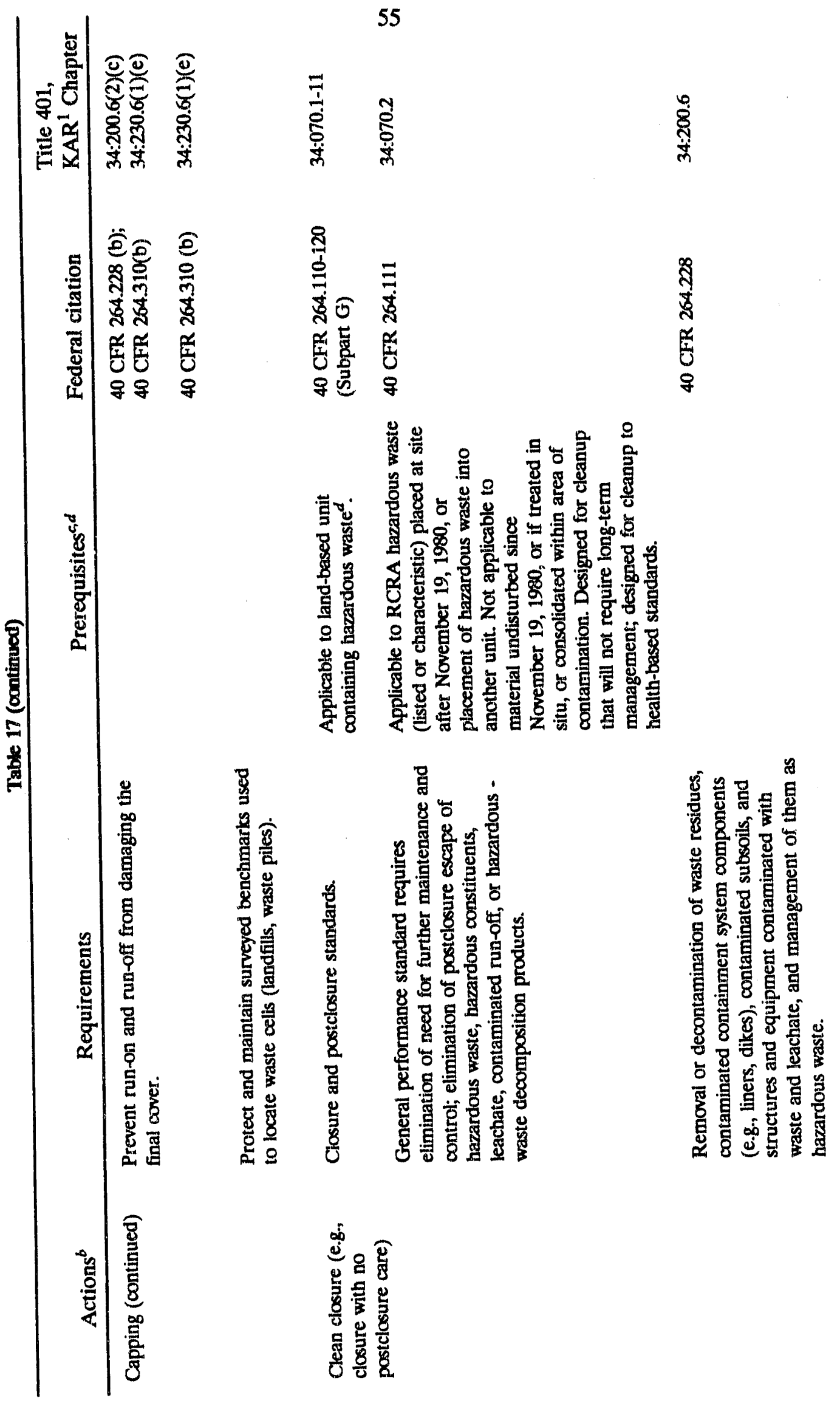




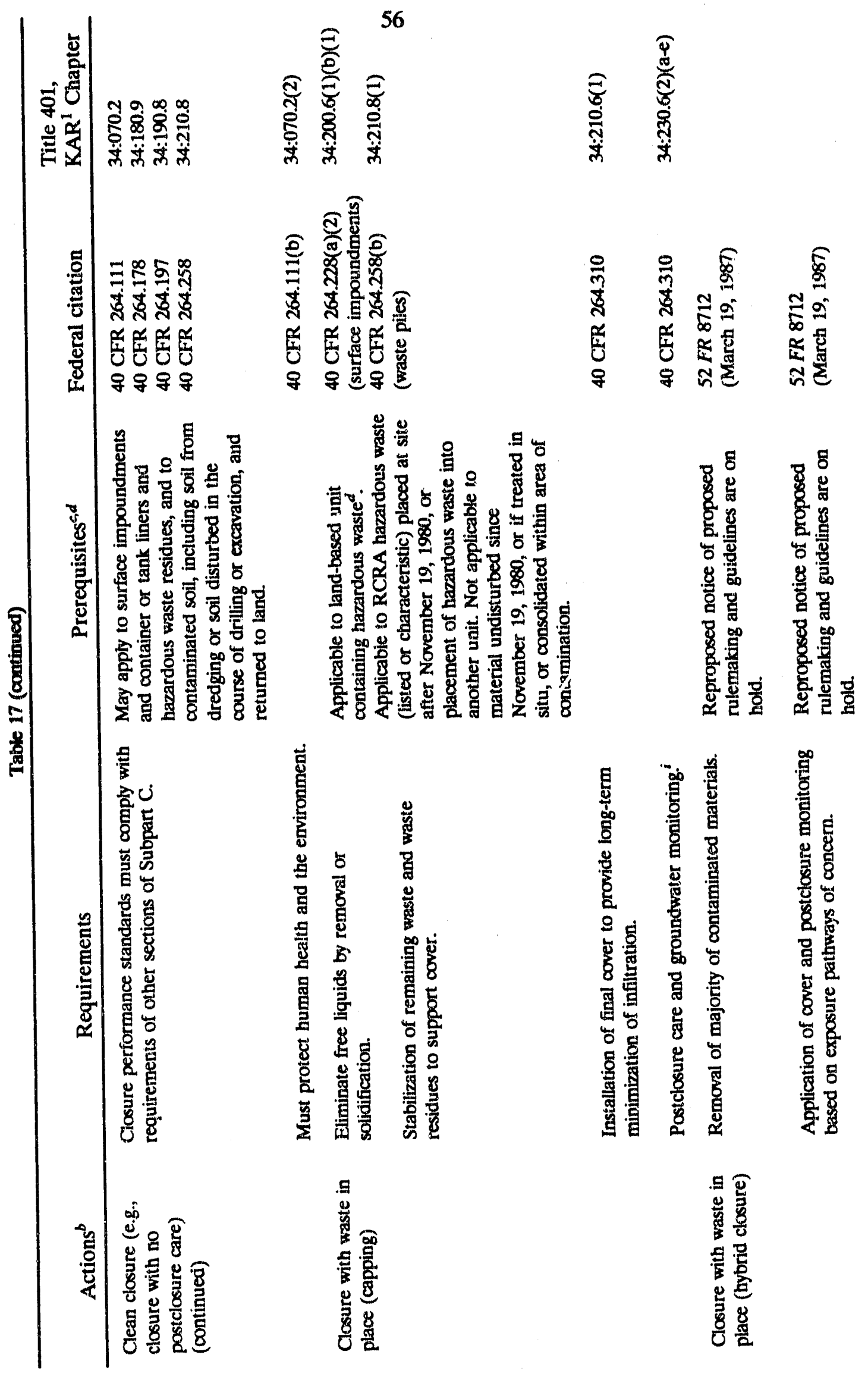




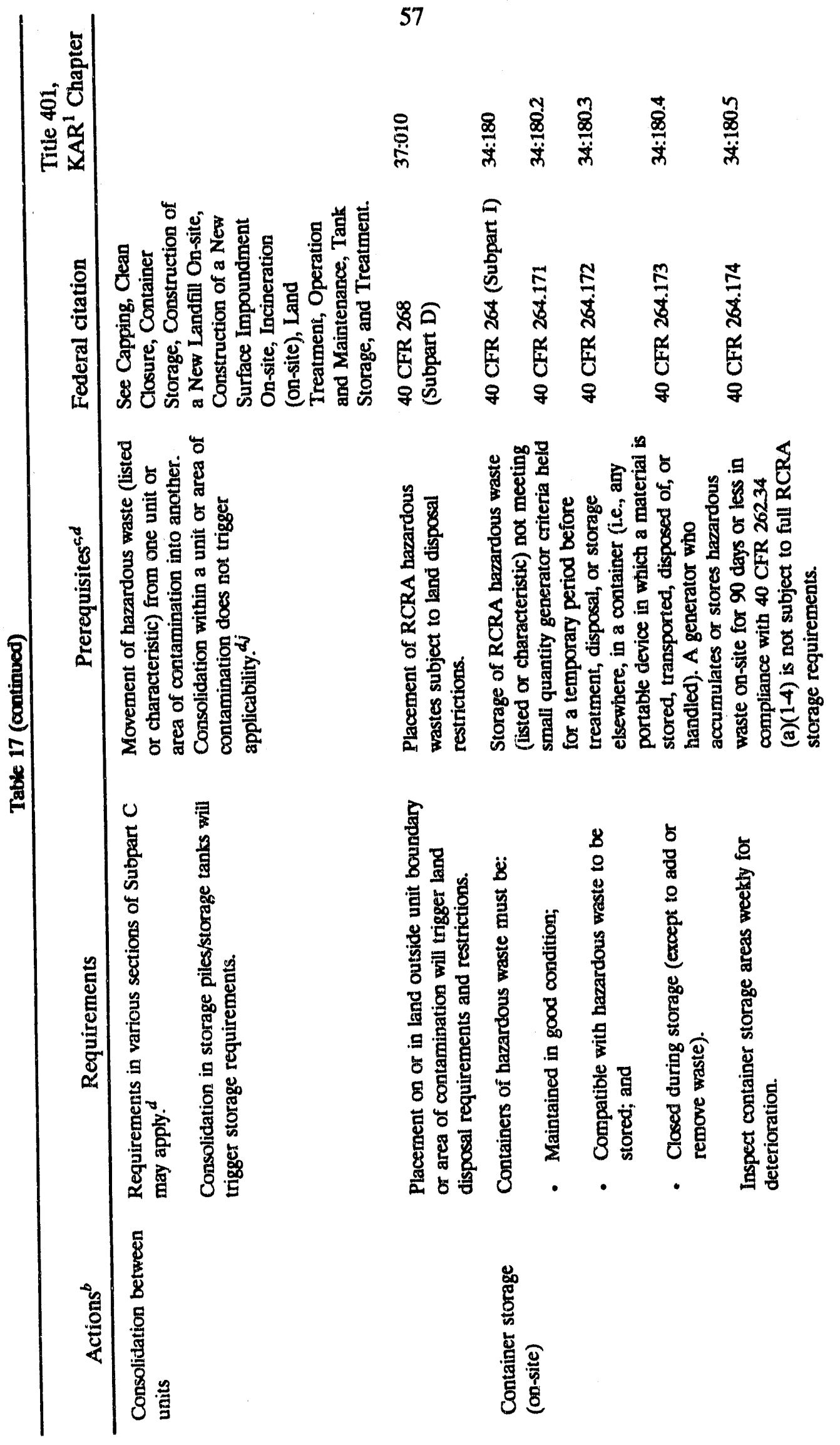




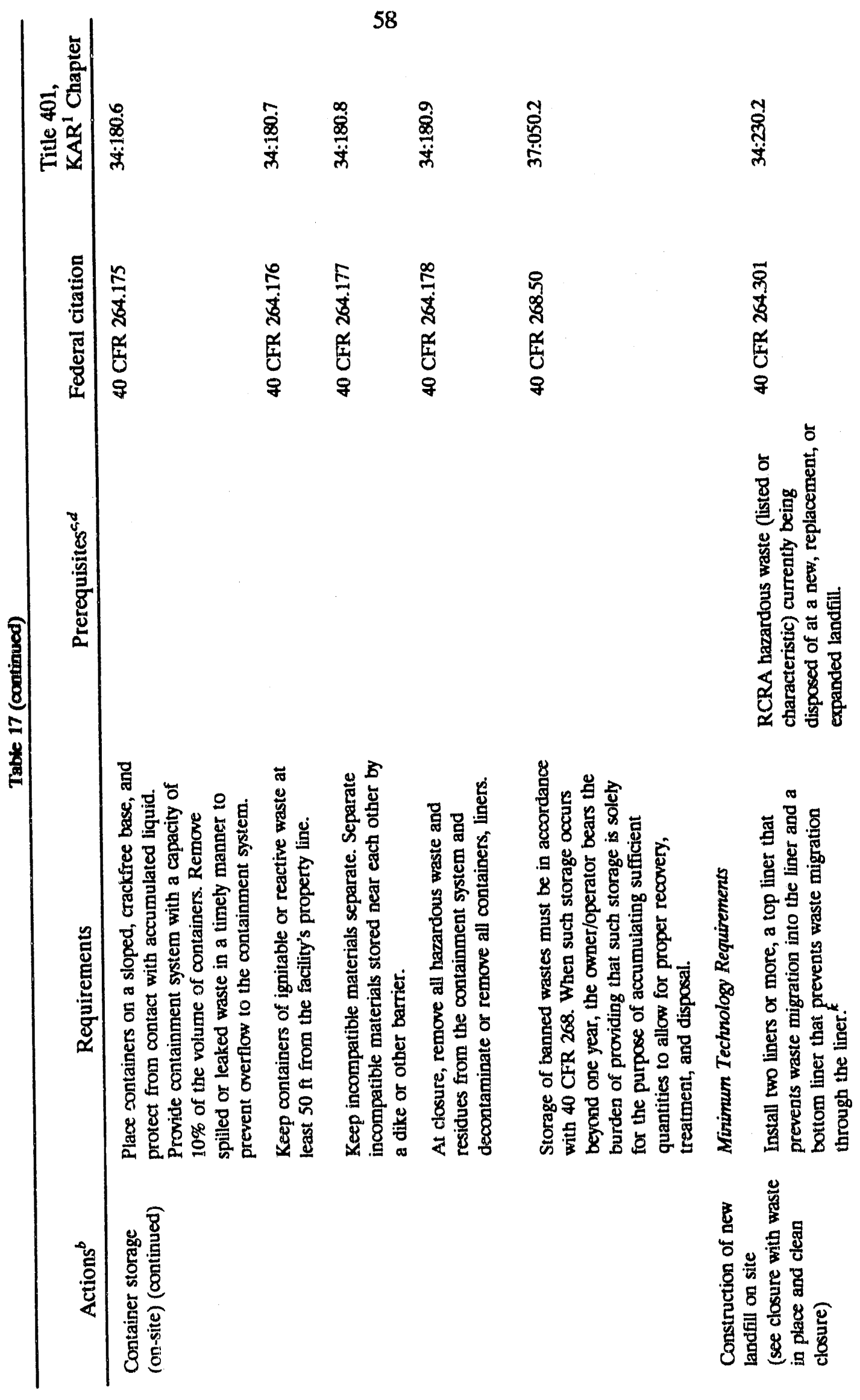




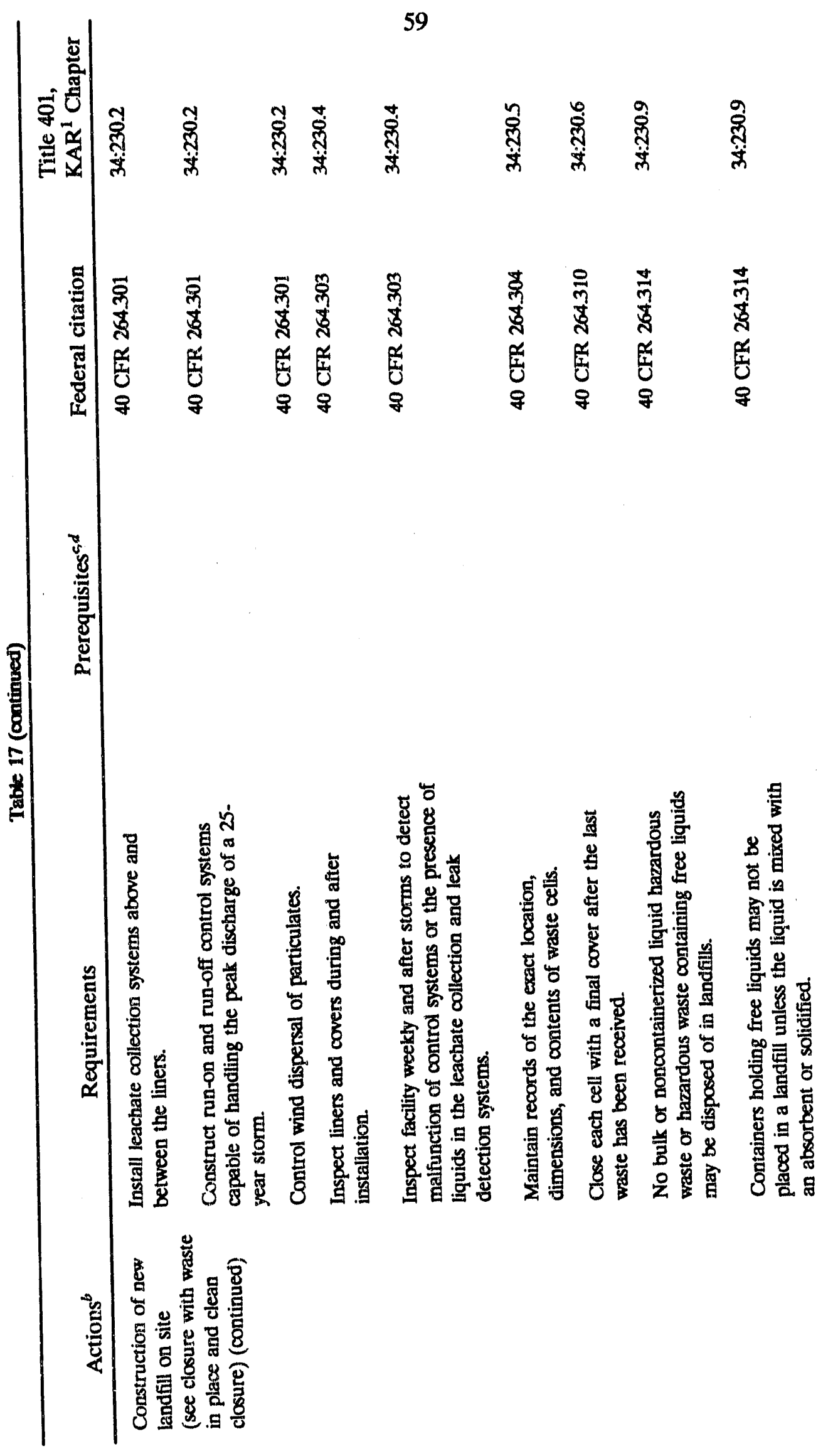




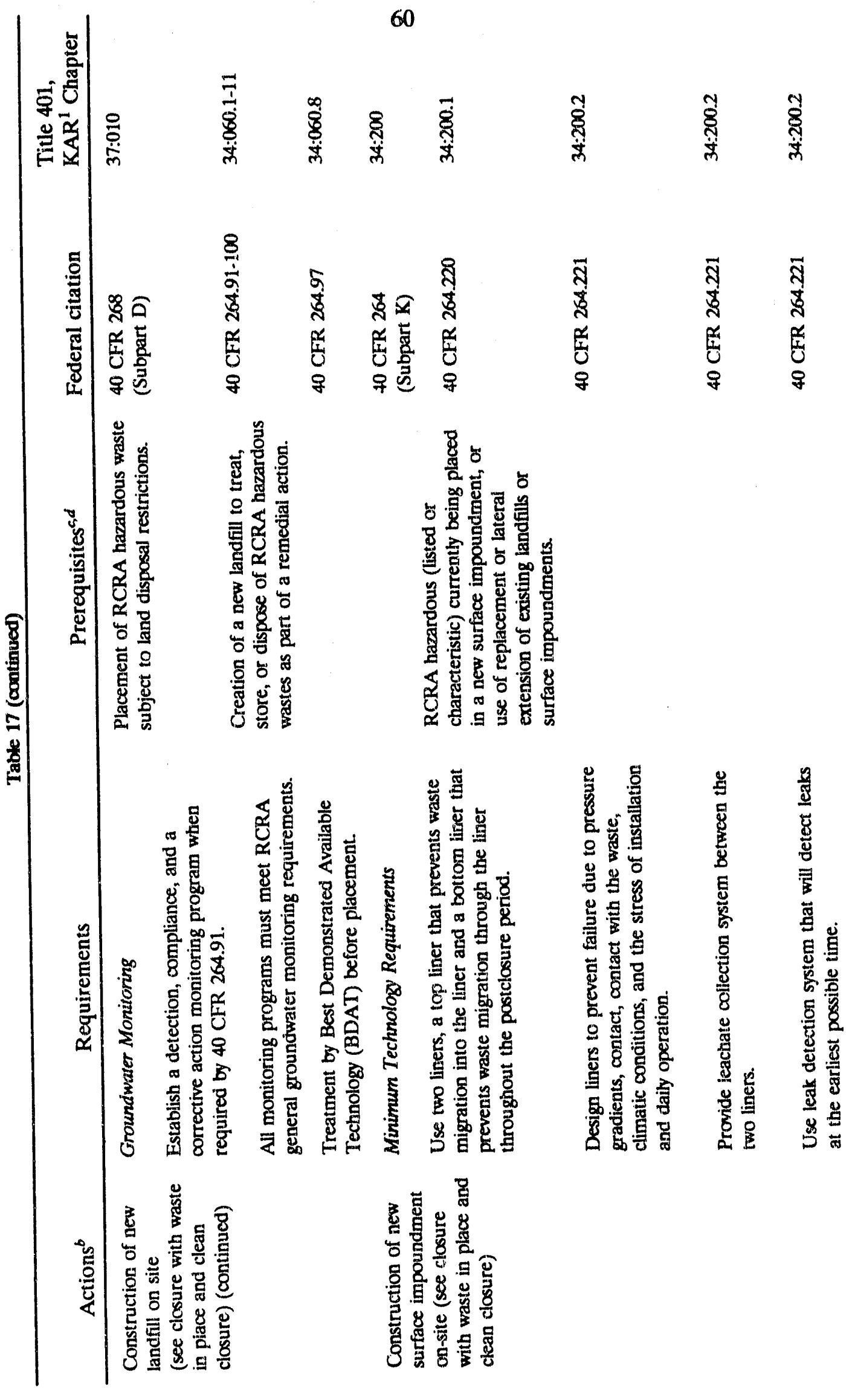




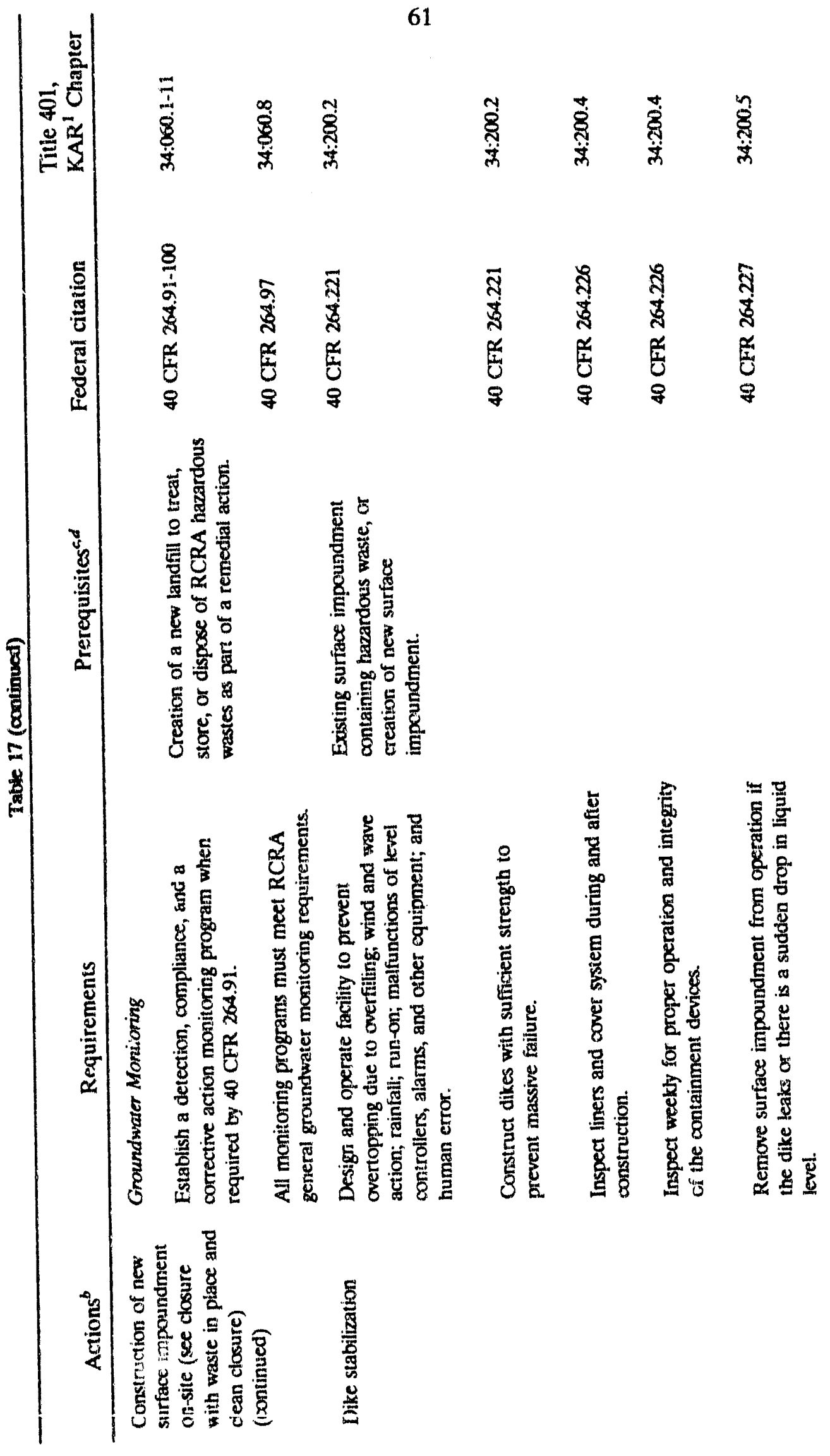




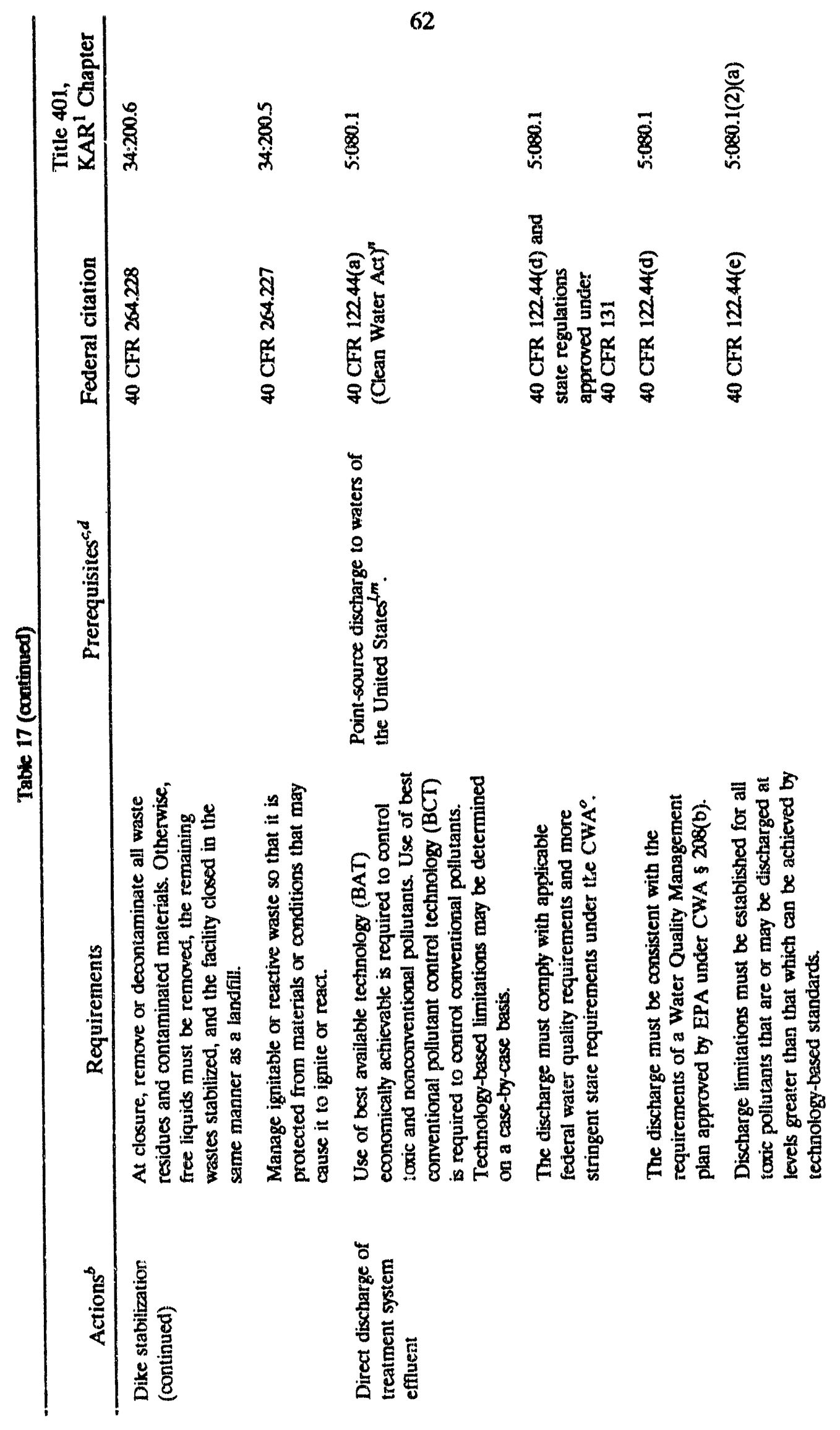




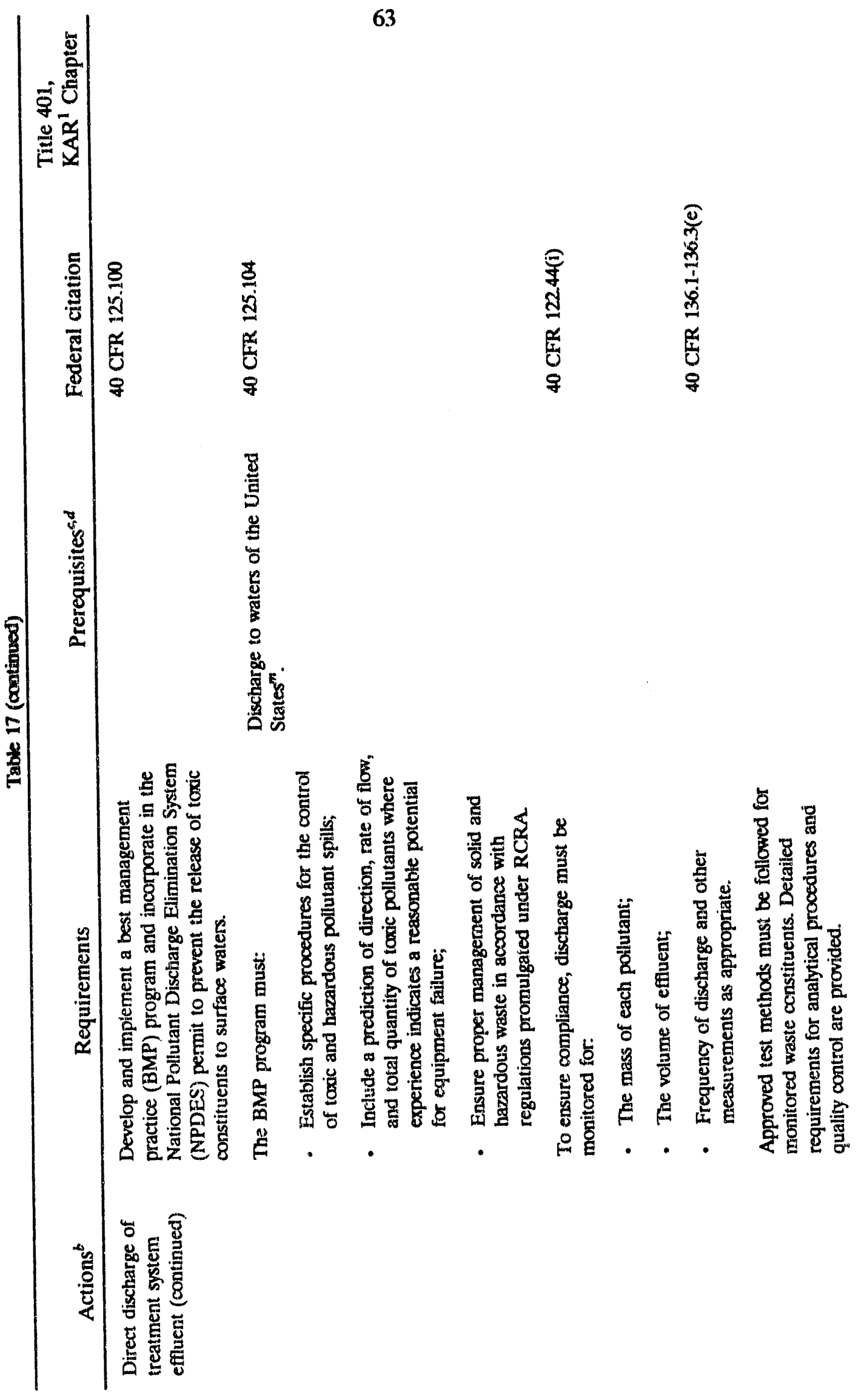




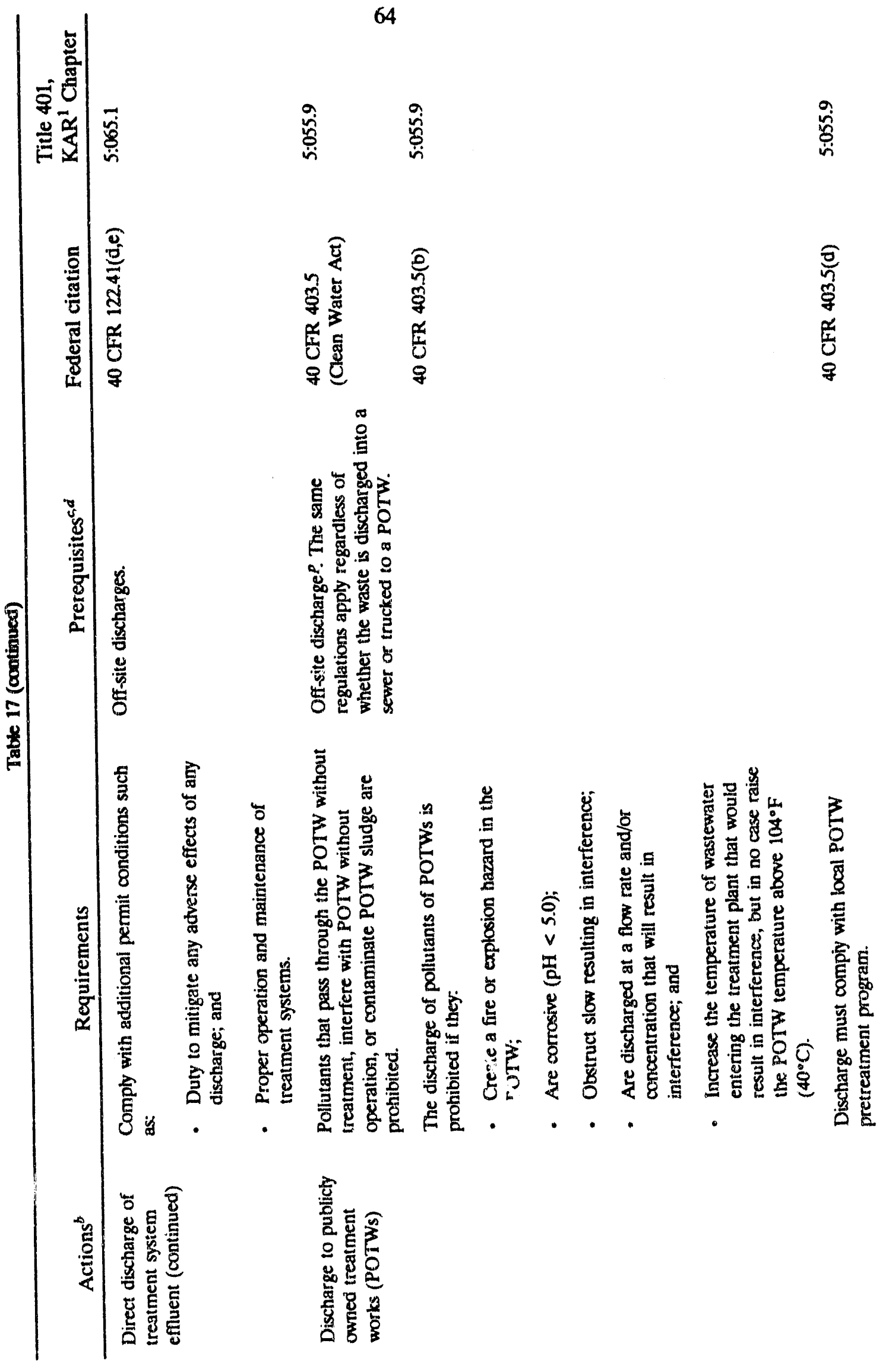




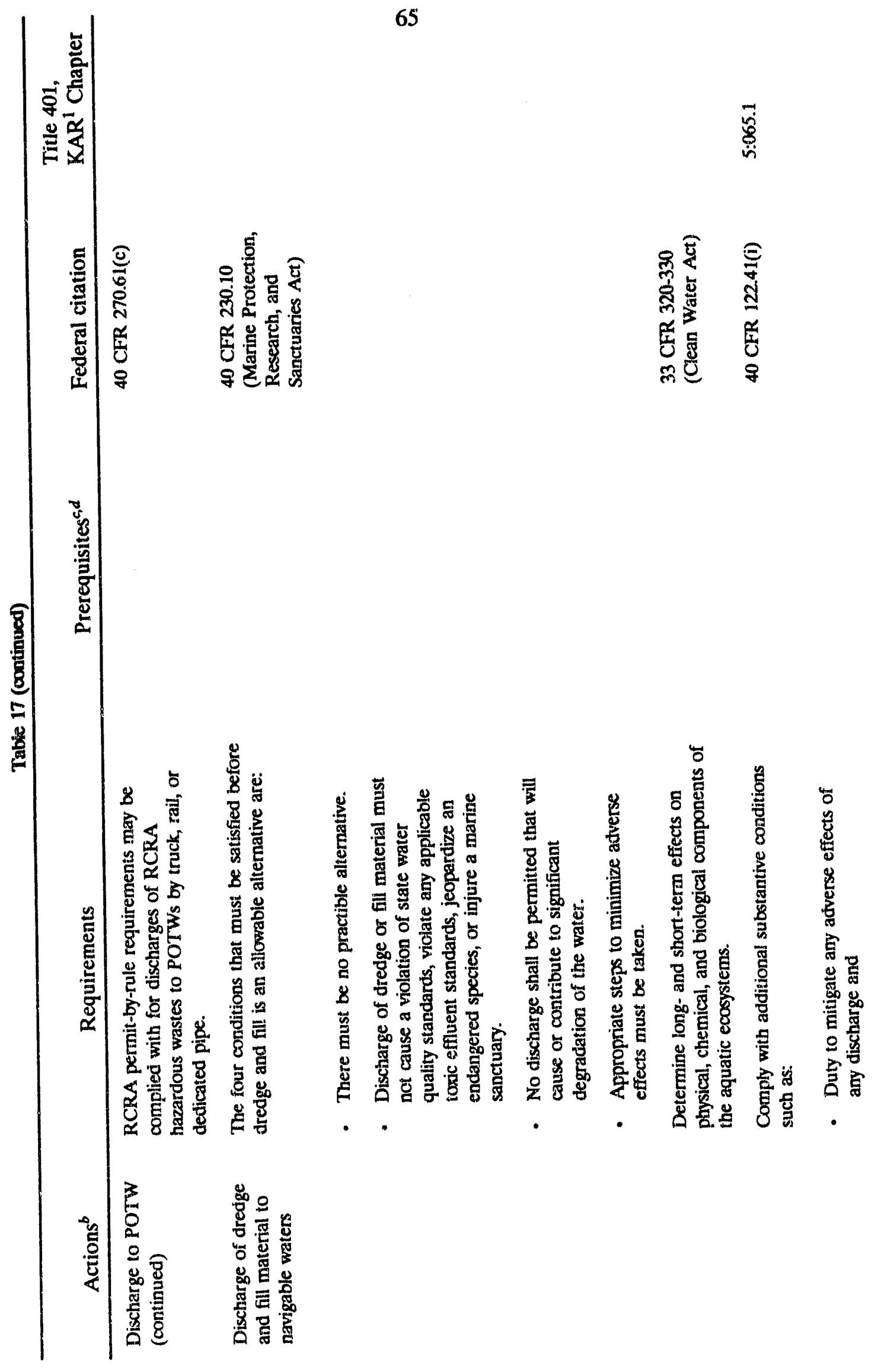




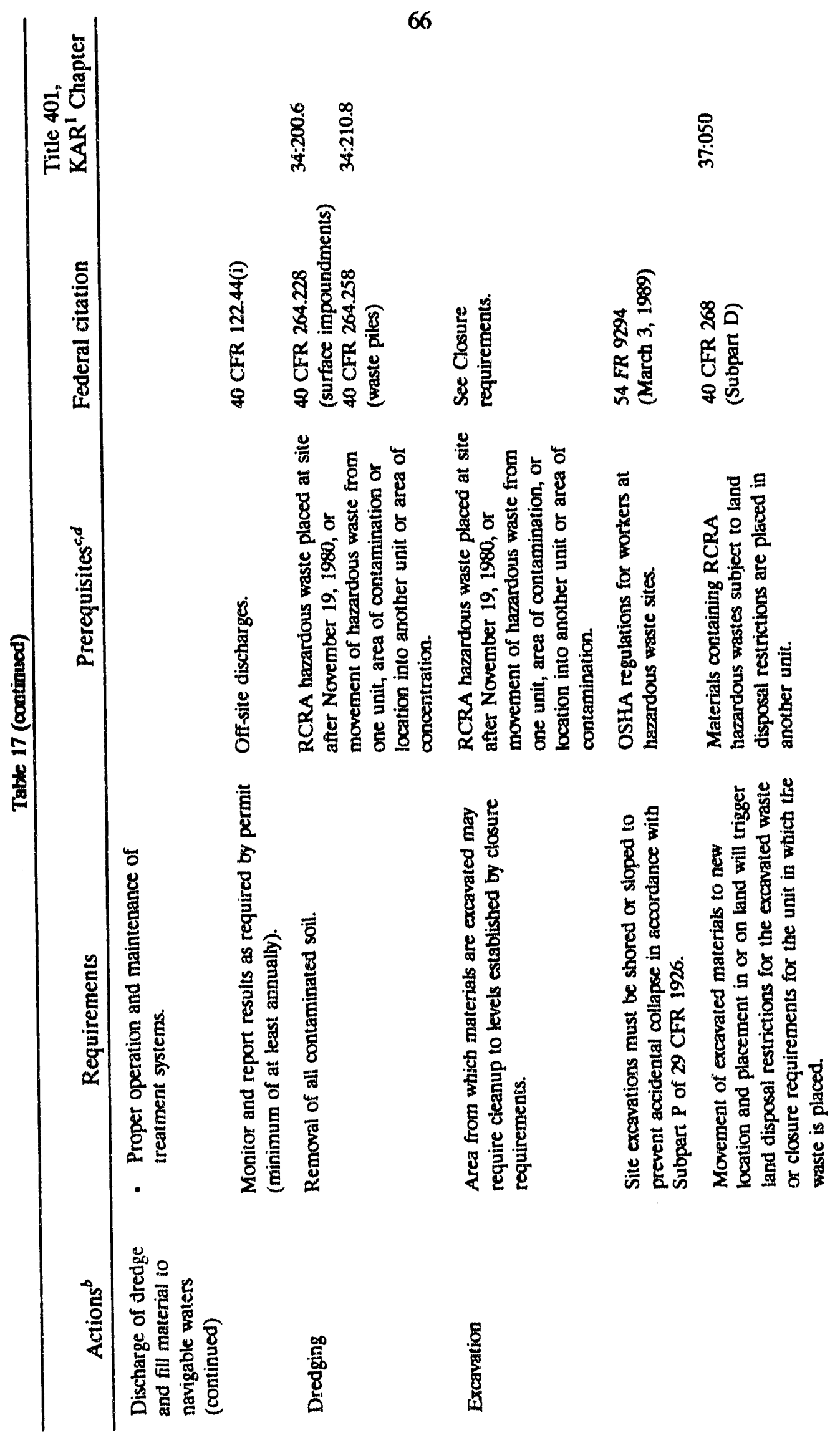




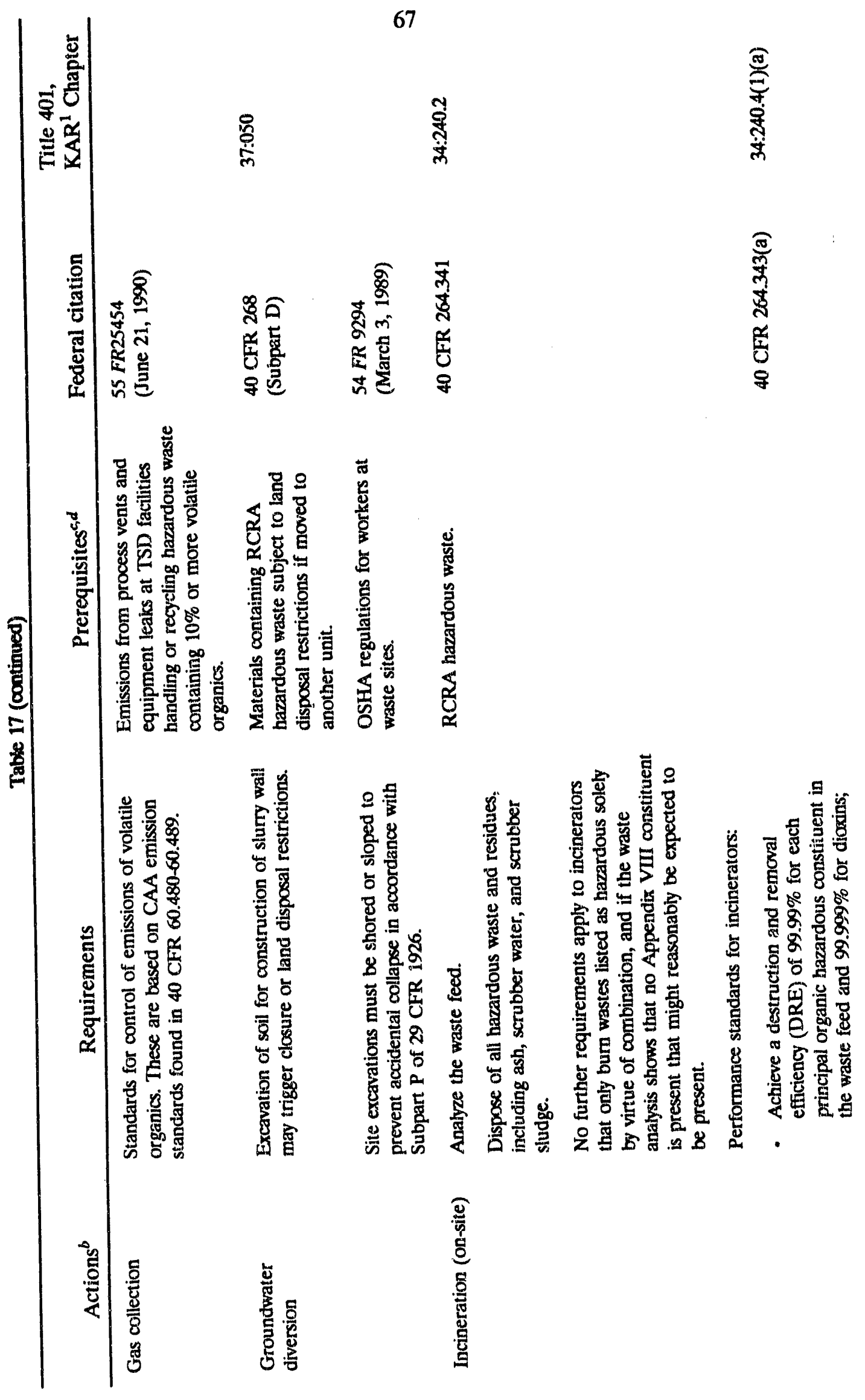




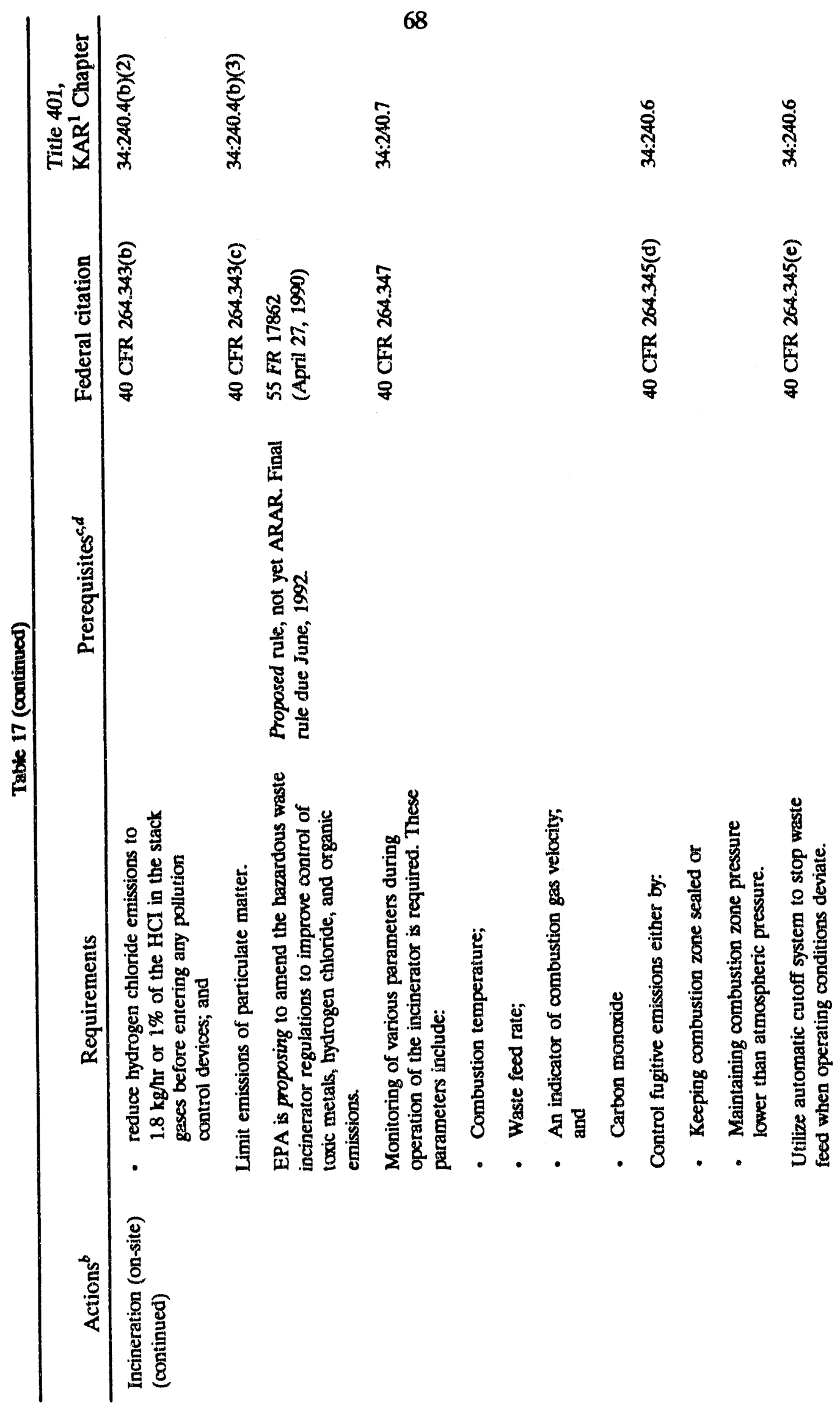




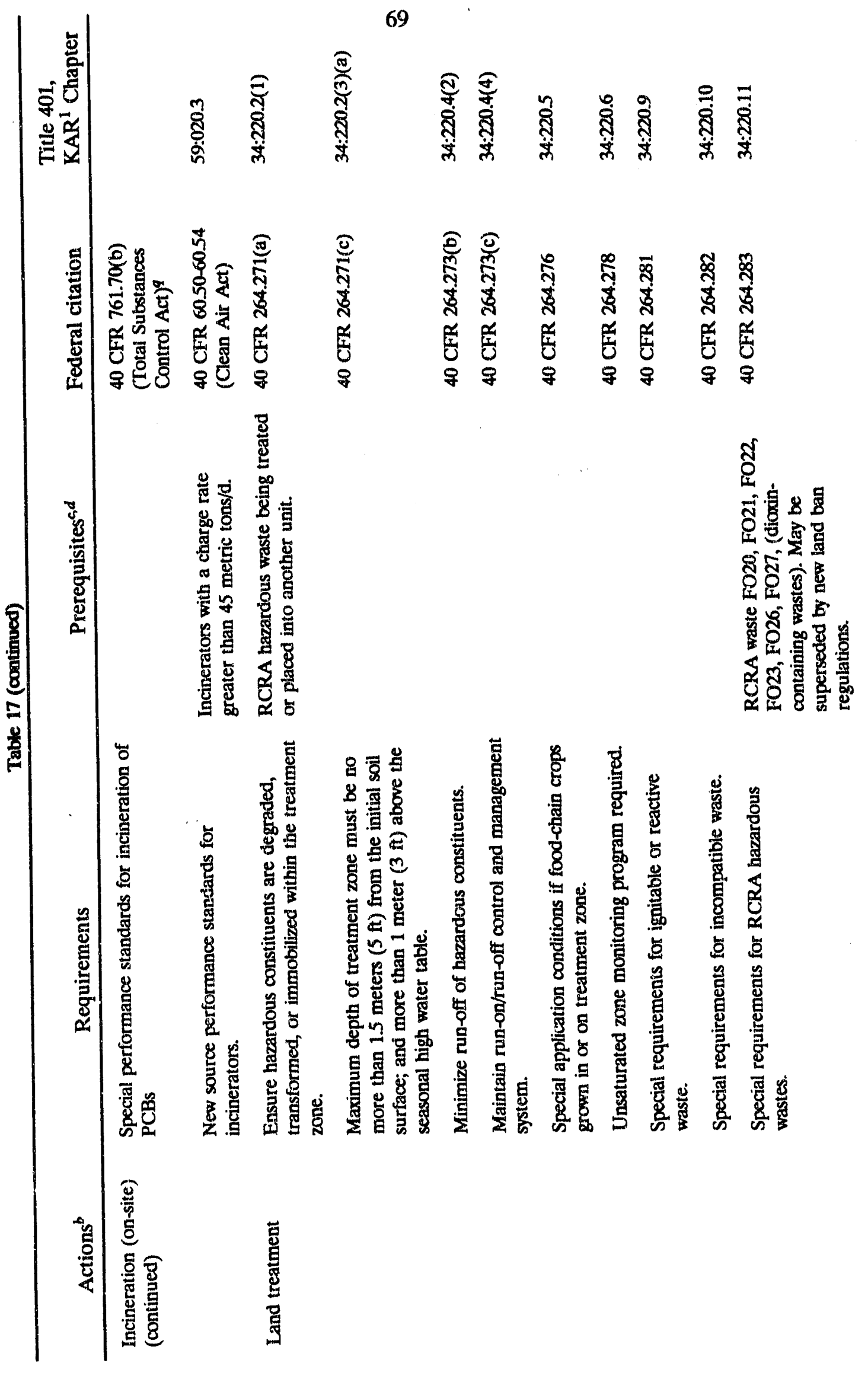




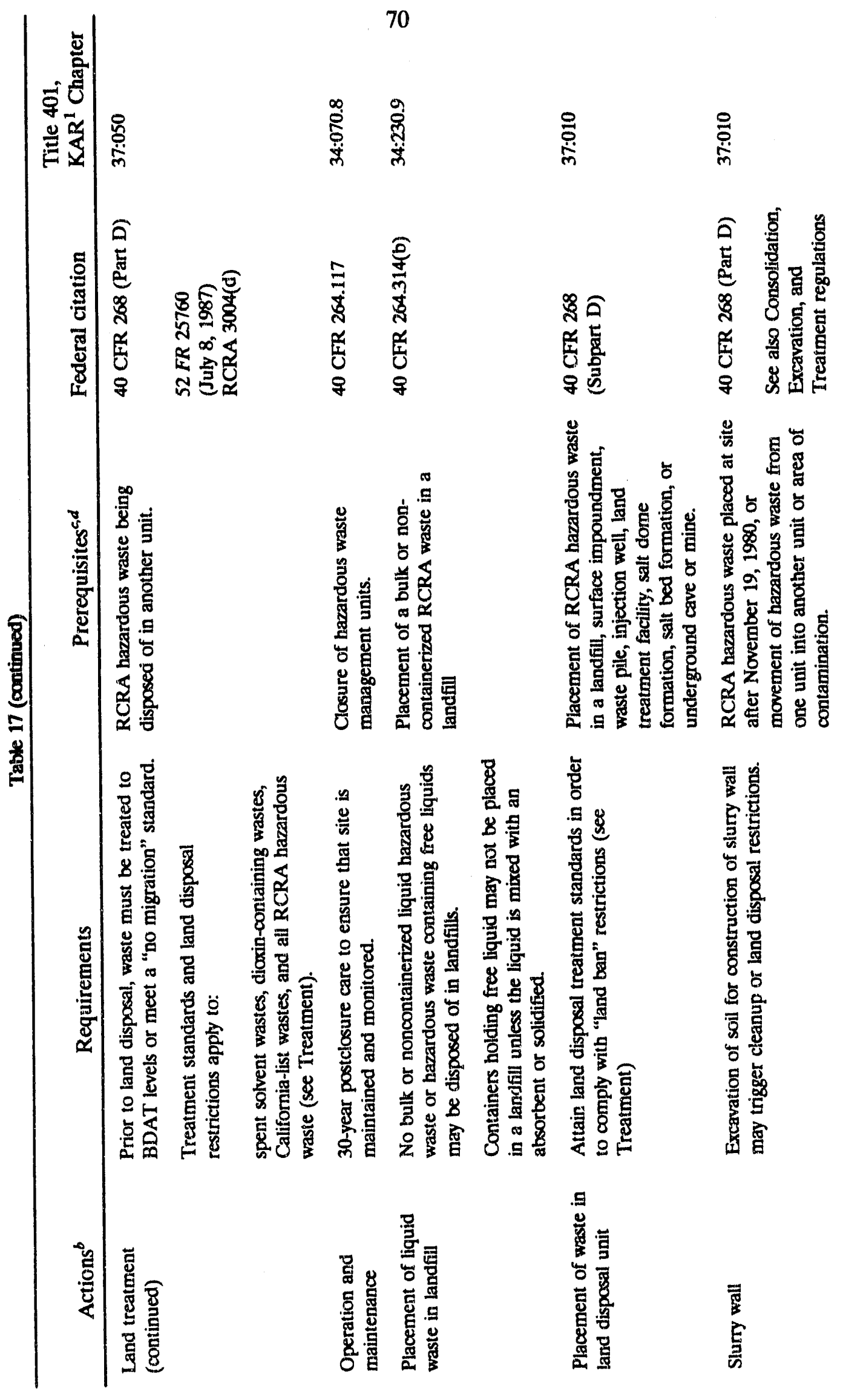




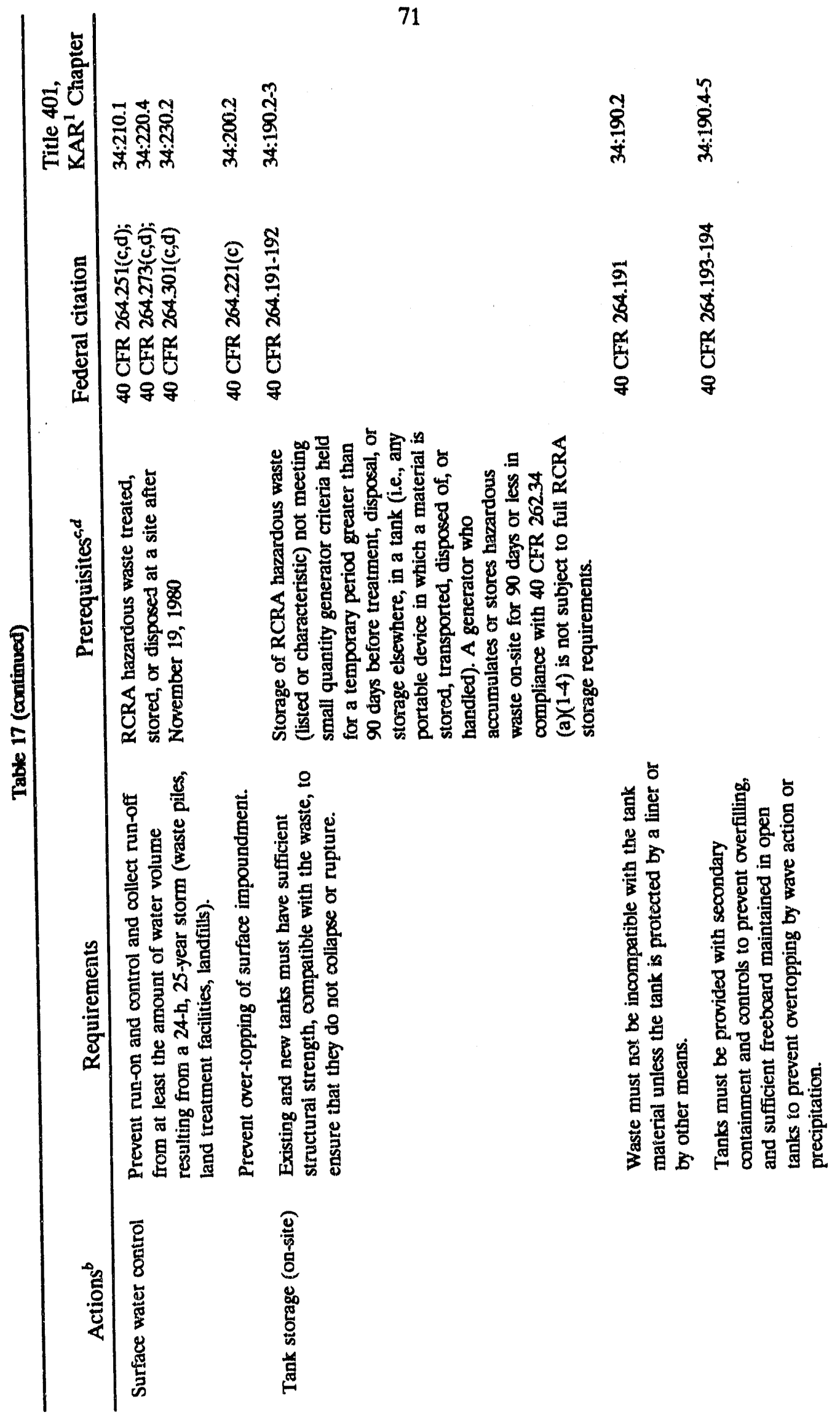




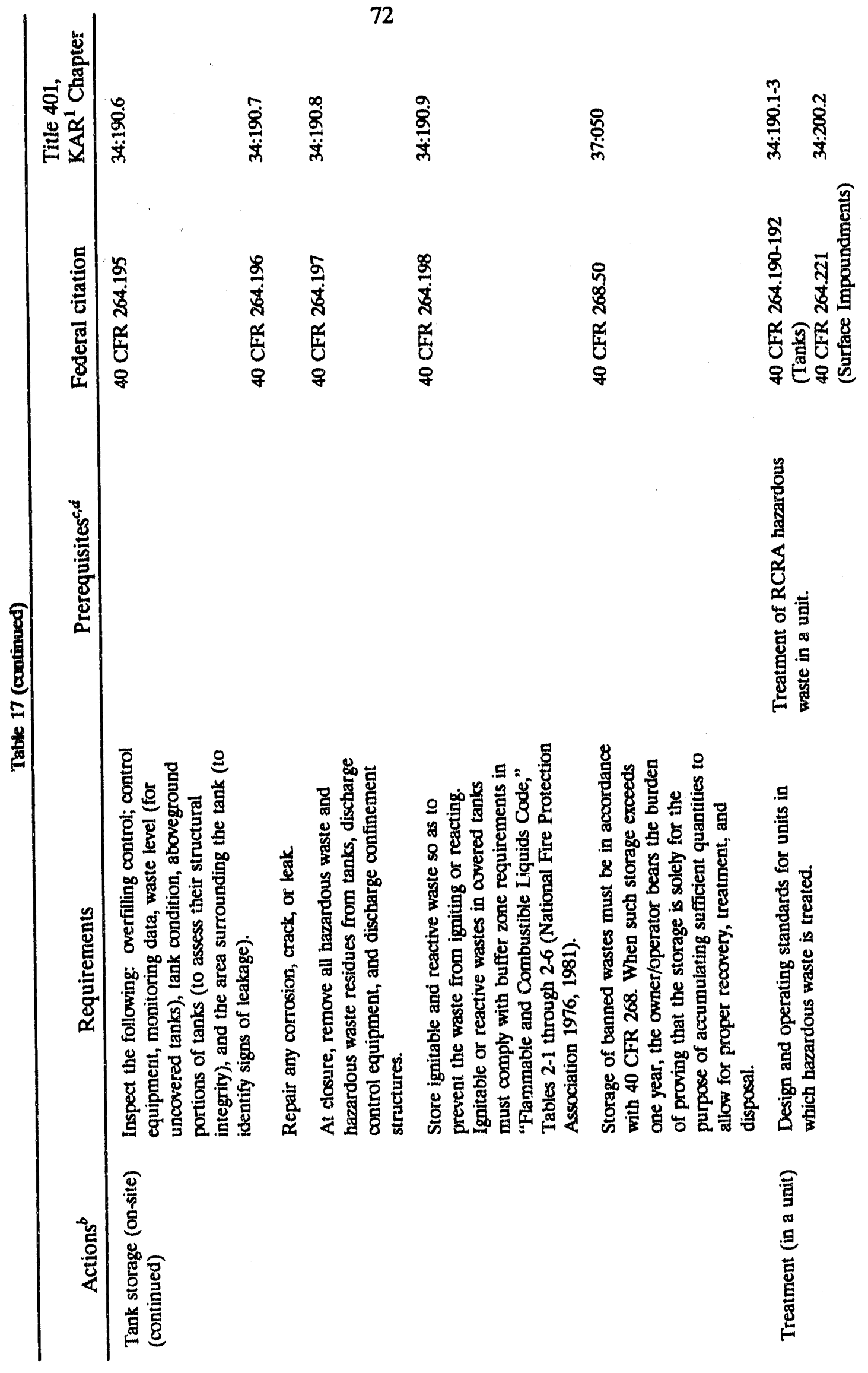




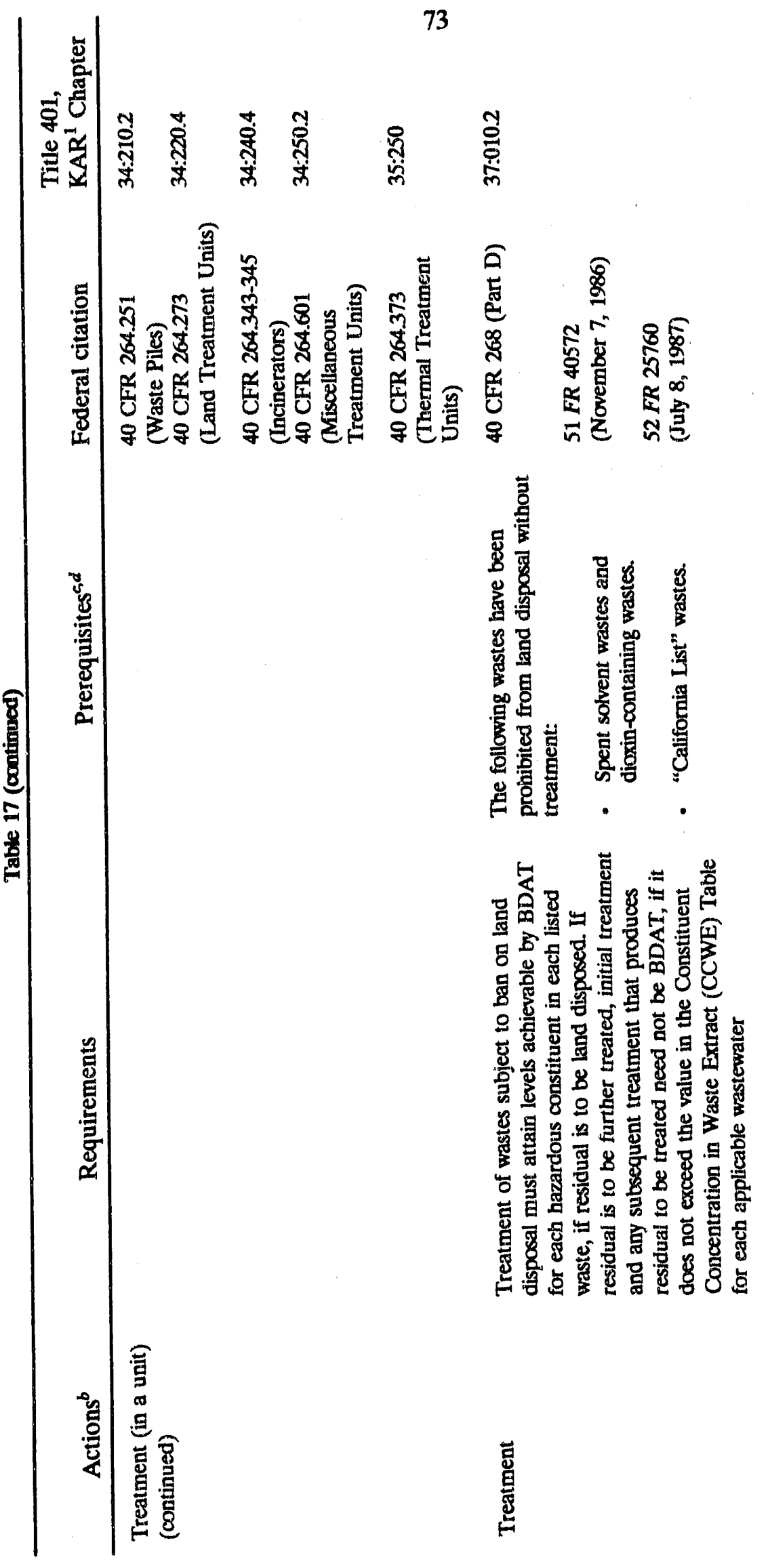




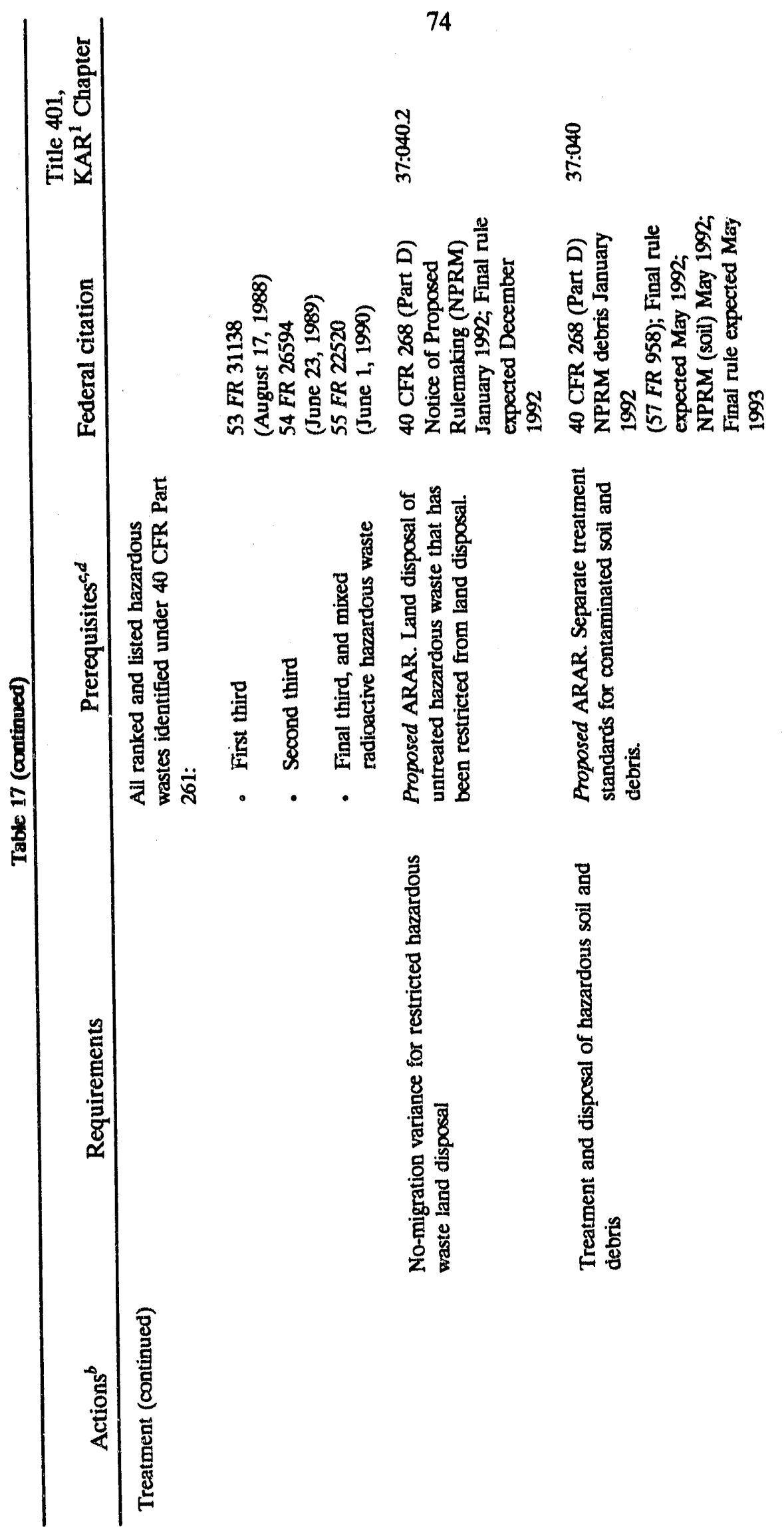




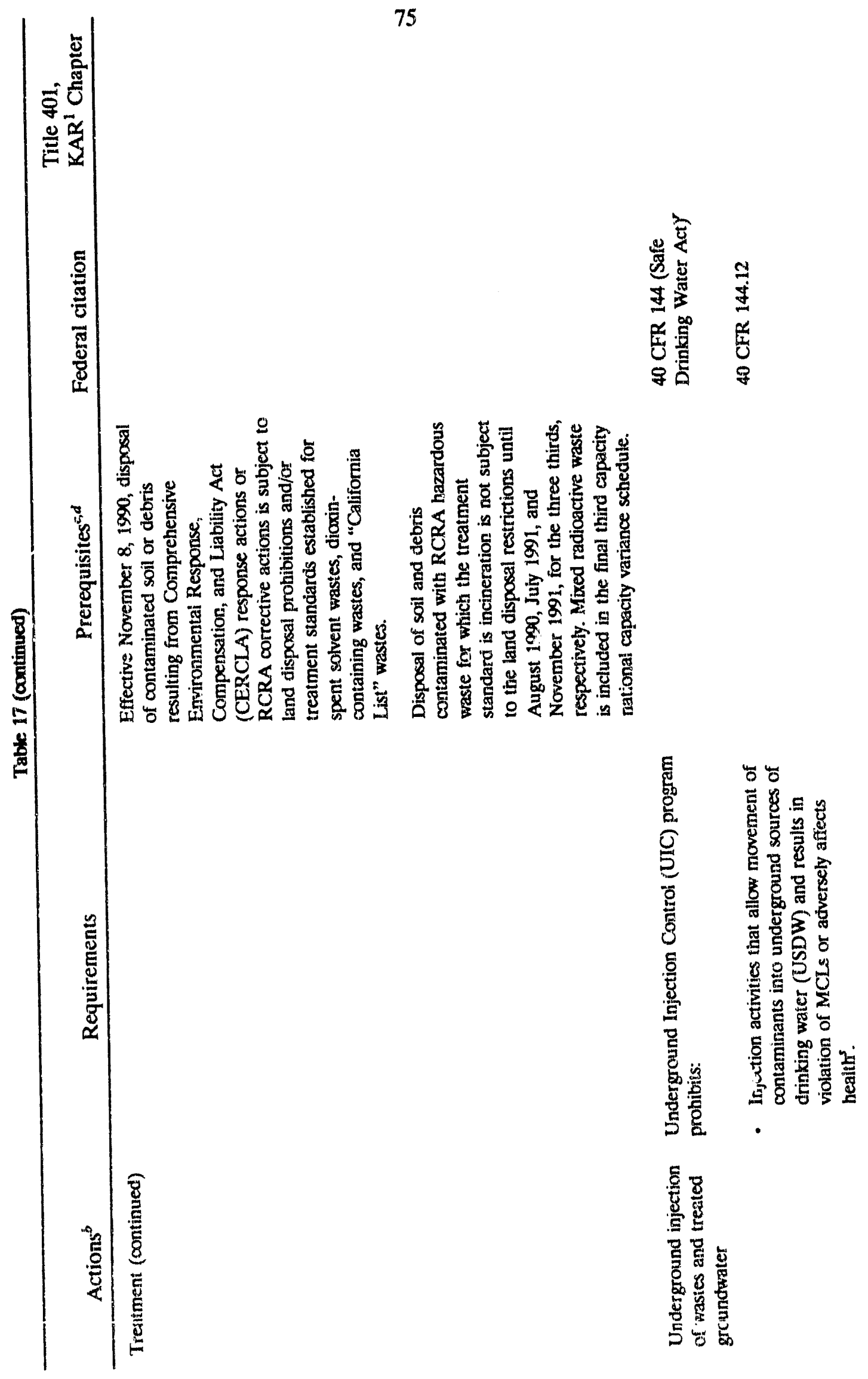




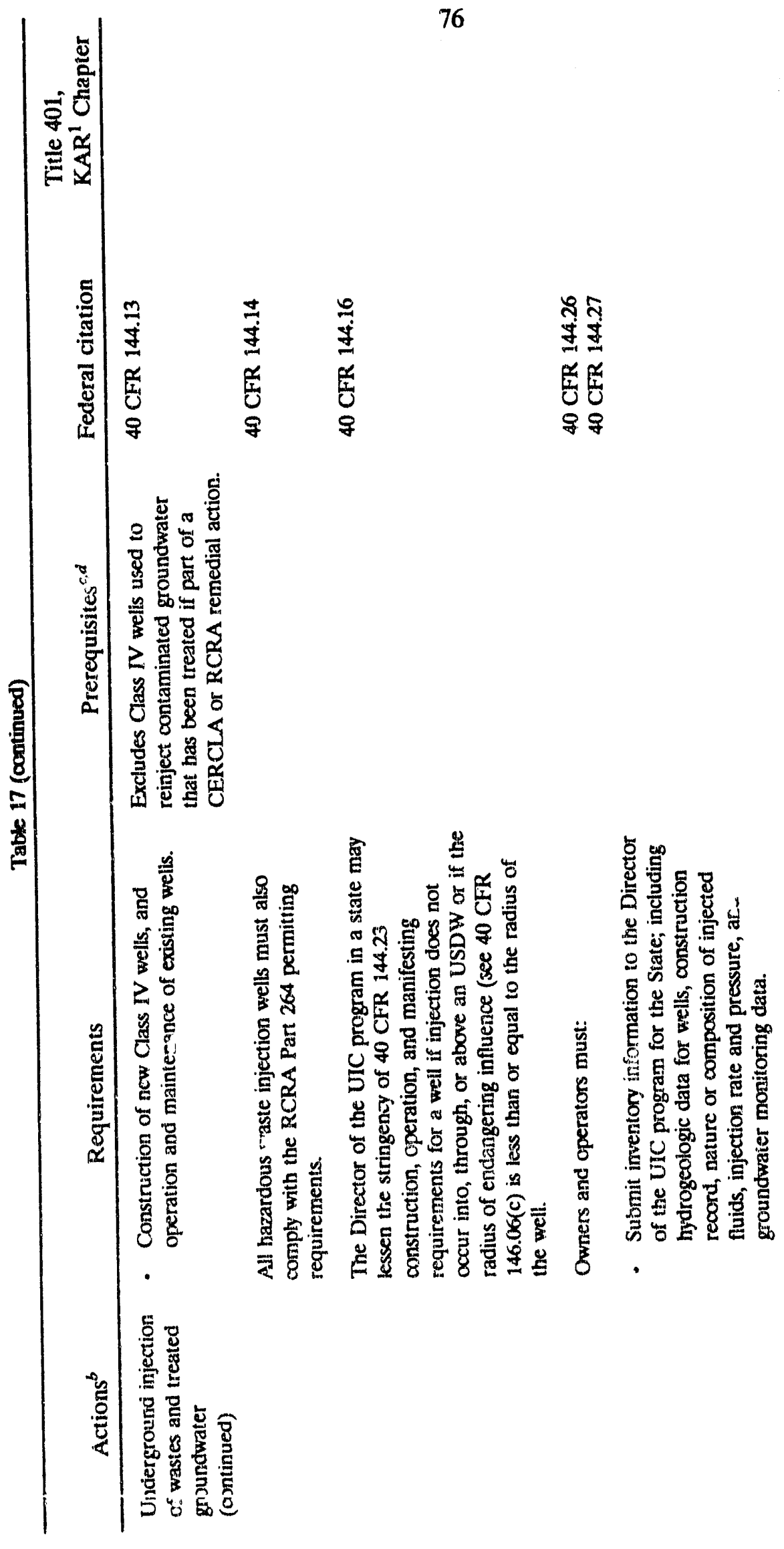




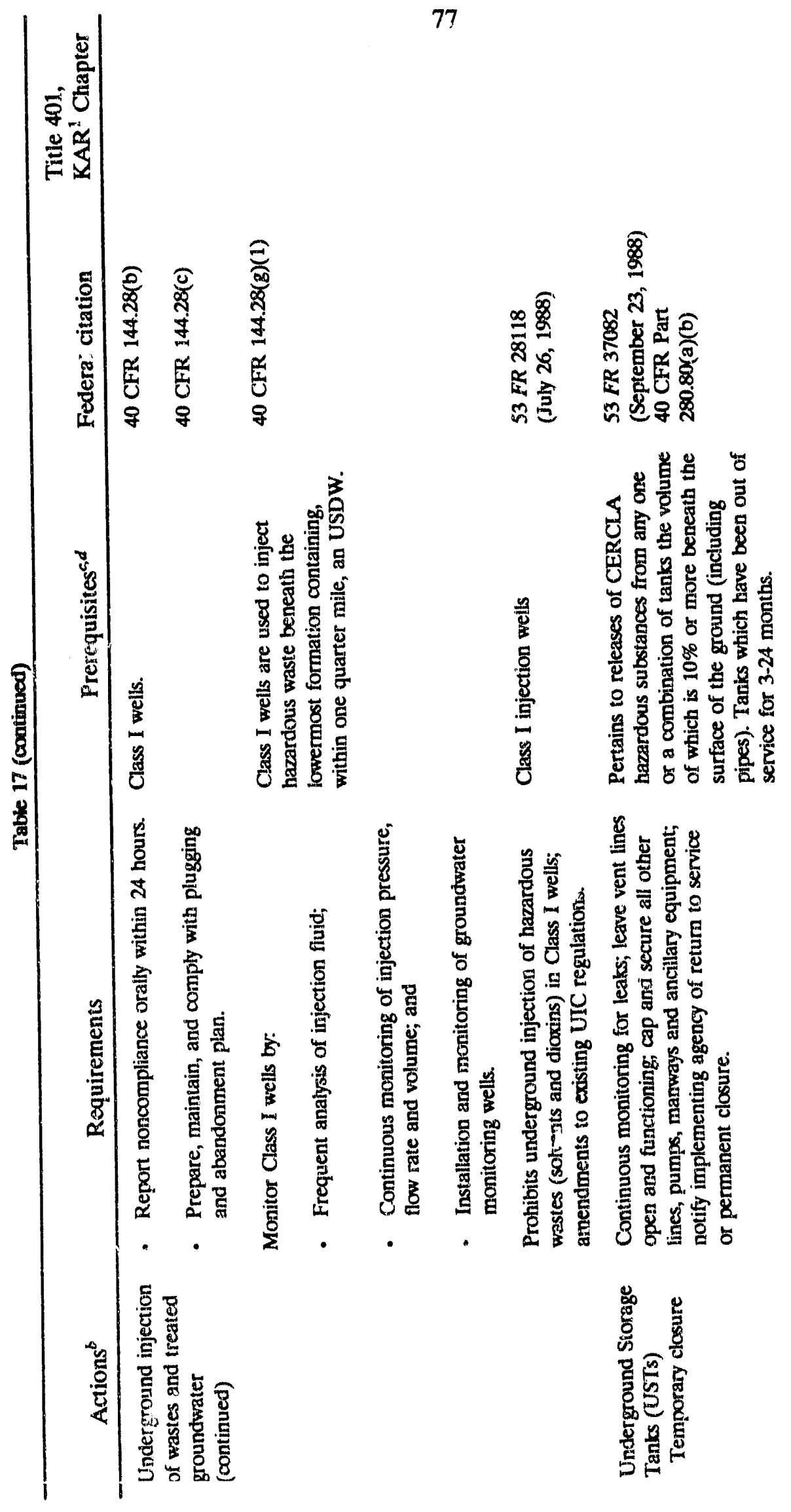




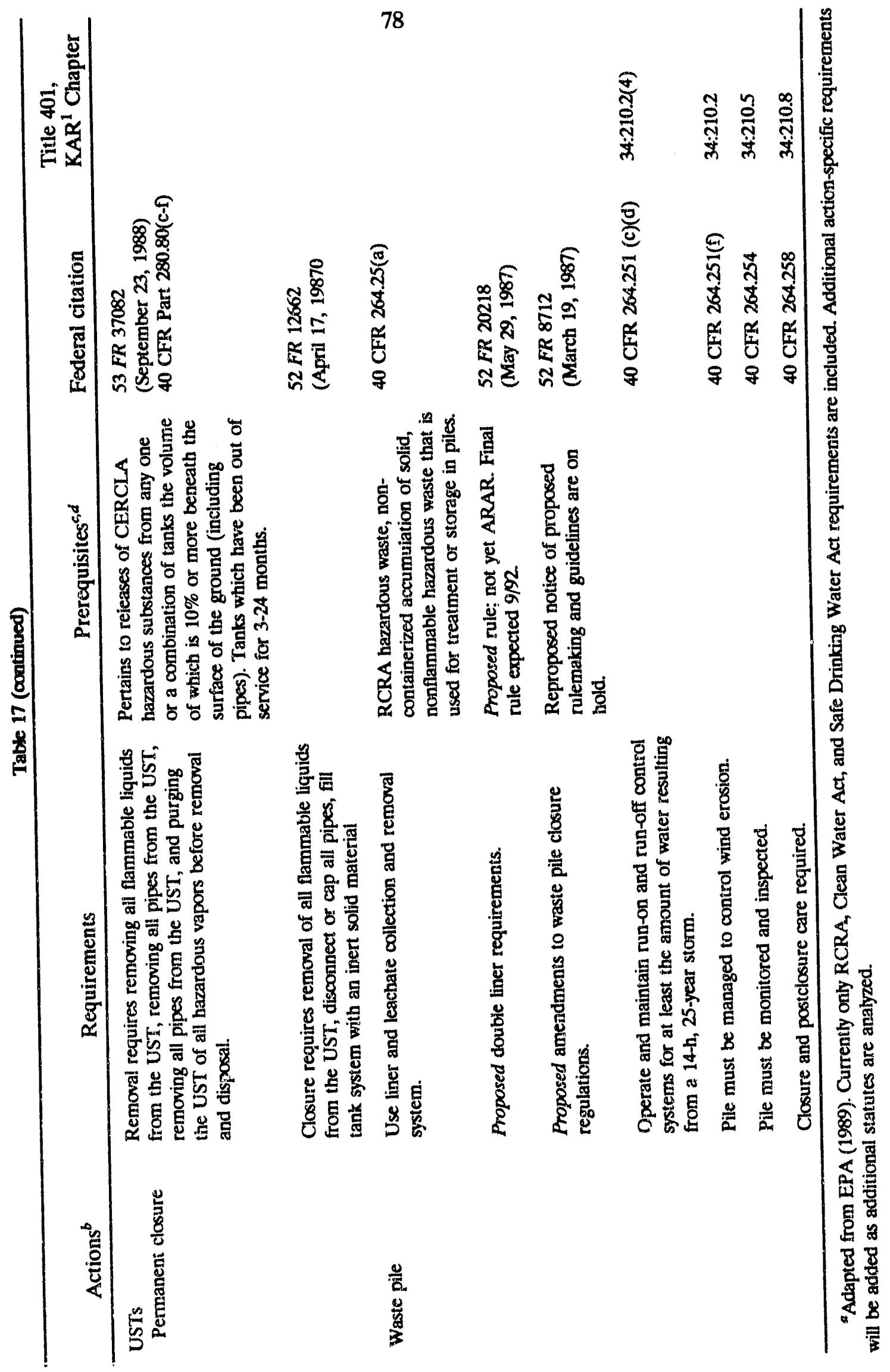




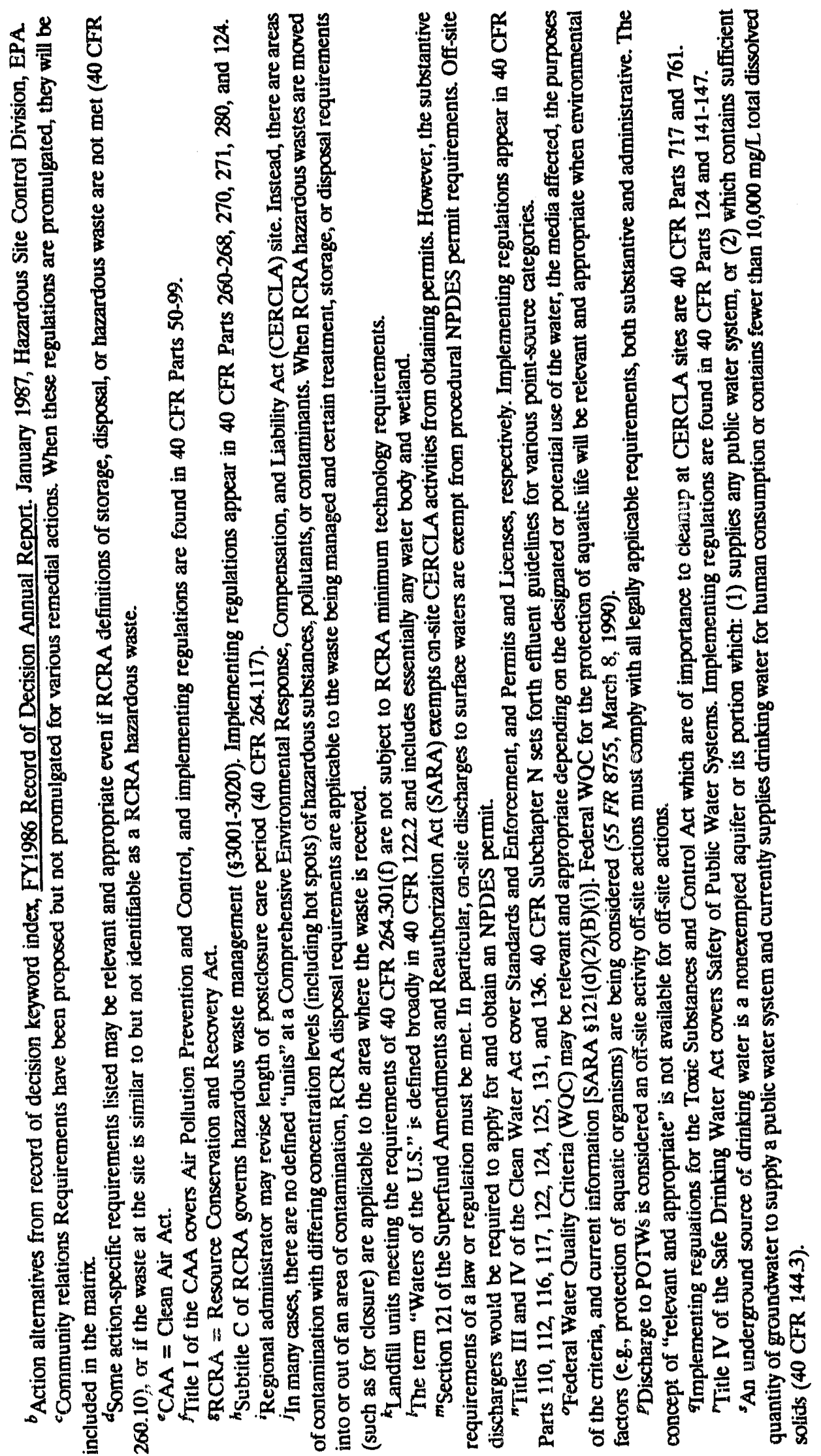




\section{REFERENCES}

Anderson, Terry. September 4, 1991. Kentucky Office of Water Quality, personal communication to E. L. Etnier.

Davis, Kenneth R. September 6, 1991. Hydrologist, Environmental Safety and Health Division, Paducah Gaseous Diffusion Plant, personal communication to L. A. Eaton.

Davis, Wayne L. September 6, 1991. Environmental Section Chief, Kentucky Department of Fish and Wildlife, personal communication to E. L. Etnier.

EPA (U.S. Environmental Protection Agency). 1988. CERCLA Compliance with Other Laws Manual, Draft Guidance. Vol. I, OSWER Directive 9234.1-01, EPA Office of Emergency and Remedial Response, Washington, D.C.

EPA (U.S. Environmental Protection Agency). 1989. CERCLA Compliance with Other Laws Manual: Part II. Clean Air Act and other Environmental Statutes and State Requirements, OSWER Directive 9234.1-02, EPA Office of Emergency and Remedial Response, Washington, D.C.

EPA (U.S. Environmental Protection Agency). August 1990. Guidance on Remedial Actions for Superfund Sites with PCB Contamination, OSWER Directive 9355.4-01, EPA Office of Emergency Remedial Response, Washington, D.C.

EPA (U.S. Environmental Proiection Agency). 1991a. Region IV Criteria Charts (October).

EPA (U.S. Environmental Protection Agency). 1991b. Integrated Risk Information System (IRIS), EPA/600/8-86/032a (current as of February 1990), EPA Office of Health and Environmental Assessment, Cincinnati, Ohio.

EPA (U.S. Environmental Protection Agency). 1991c. Health Effects Assessment Summary Tables. First Quarter, FY 1991, OERR 9200.6-303-(91-1), EPA Office of Emergency and Remedial Response, Washington, D.C.

Etnier, D. A. December 4, 1991. Professor of Zoology, University of Tennessee, Knoxville, Tennessee, personal communication to E. L. Etnier.

Government Institutes, Inc. 1991. Kentucky Environmental Law Handbook, Rockville, Maryland.

Hendricks, William September 10, 1991. Environmental Biologist, Kentucky State Nature Preserves Commission, database September 1991, personal communication to E. L. Etnier.

Houlberg, L. M., G. T. Hawkins, M. S. Salk December 1991. Environmental Regulatory Update Table, July, ORNL-6658/R11, Martin Marietta Energy Systems, Inc., Oak Ridge National Laboratory.

Jeffs, Vicki September 6, 1991. Kentucky Radiation Control Branch, Radioactive Materials Section, personal communication to E. L. Etnier. 
Jones, Vicki August 30, 1991a. National Environmental Policy Act (NEPA) Coordinator, Paducah Gaseous Diffusion Plant, Paducah, Kentucky, personal communication to L. A. Eaton.

Jones, Vicki December 4, 1991b. National Environmental Policy Act (NEPA) Coordinator, Paducah Gaseous Diffusion Plant, Paducah, Kentucky, personal communication to E. L. Etnier.

Kentucky Academy of Sciences, Kentucky Nature Preserves Commission (KAS-KNPC) 1986. "Endangered, Threatened, and Rare Plants and Animals of Kentucky," Trans. Kentucky Acad. Sci. 47:83-97.

McCracken, Gary September 6, 1991. Department of Zoology, University of Tennessee, personal communication to L. A. Eaton.

Ryon, M. G. October 1, 1991. Environmental Sciences Division, Oak Ridge National Laboratory, personal communication to $\mathrm{E}$. L. Etnier.

Schenian, Pam September 6, 1991. Murray State Archeology Department, Murray, Kentucky, personal communication to L. A. Eaton.

Schiefferle, Ralpi September 4, 1991. Kentucky Office of Drinking Water, personal communication to L. A. Eaton.

Schneider, Clifford September 10, 1991. Kentucky Office of Water Quality, personal communication to E. L. Etnier.

Taylor, Tuss September 6, 1991 and October 24, 1991. Kentucky Division of Waste Management, personal communication to E. L. Etnier. 
KY/ER-8

\section{DISTRIBUTION}

1. L. V. Asplund

2. L. D. Bates

3. C. A. Belnatz

4. L. B. Cobb

5. D. G. Cope

6. M. F. P. DeLozier

7. N. W. Durfee

8. L. A. Eaton

9-12. E. L. Etnier

13. M. W. Francis

14. C. G. Giltner

15. C. D. Goins

16. L. M. Houlberg

17. C. A. Hudson

18. K. G. Kahl

19. S. Leone

20. P. Y. Lu
21. J. A. Martin

22. J. C. Massey

23. J. Maudlin

24. E. P. McDonald

25. B. J. Montgomery

26. P. T. Owen

27. S. Polston

28. H. Pulley

29. R. H. Ross

30. G. E. Rymer

31. D. W. Swindle

32. R. K. White

33. K. R. Stokes

34. C. L. Young

35. Paducah Library

36. ER Document Management Center

37-41. Paducah Records Retention

42. R. C. Edwards, Department of Energy Oak Ridge Field Office, Paducah, Kentucky, Building C100, DOE Office

43-46. W. E. Murphie, Department of Energy, Office of Environmental Restoration, Eastern Area D\&D Branch, EM-423 (GTN), Washington, DC 20545

47-66. R. C. Sleeman, Department of Energy Oak Ridge Field Office, P.O. Box 2001, Oak Ridge, TN 37831-8541

67-68. D. C. Booher, Department of Energy Oak Ridge Field Office, Paducah, Kentucky, Building C100, DOE Office

69. John Young, Camp Dresser McKee, 701 Scarboro Road, Suite 3005, Oak Ridge, TN 37830

70. David E. Grambrell, MK Ferguson, P.O. Box 2001, Oak Ridge, TN 37831-2001

71. David L. Jones, EBASCO, 111 Union Valley Road, Oak Ridge, TN 37830

72-73. Office of Scientific and Technical Information, P.O. Box 62, Oak Ridge, TN 37831 

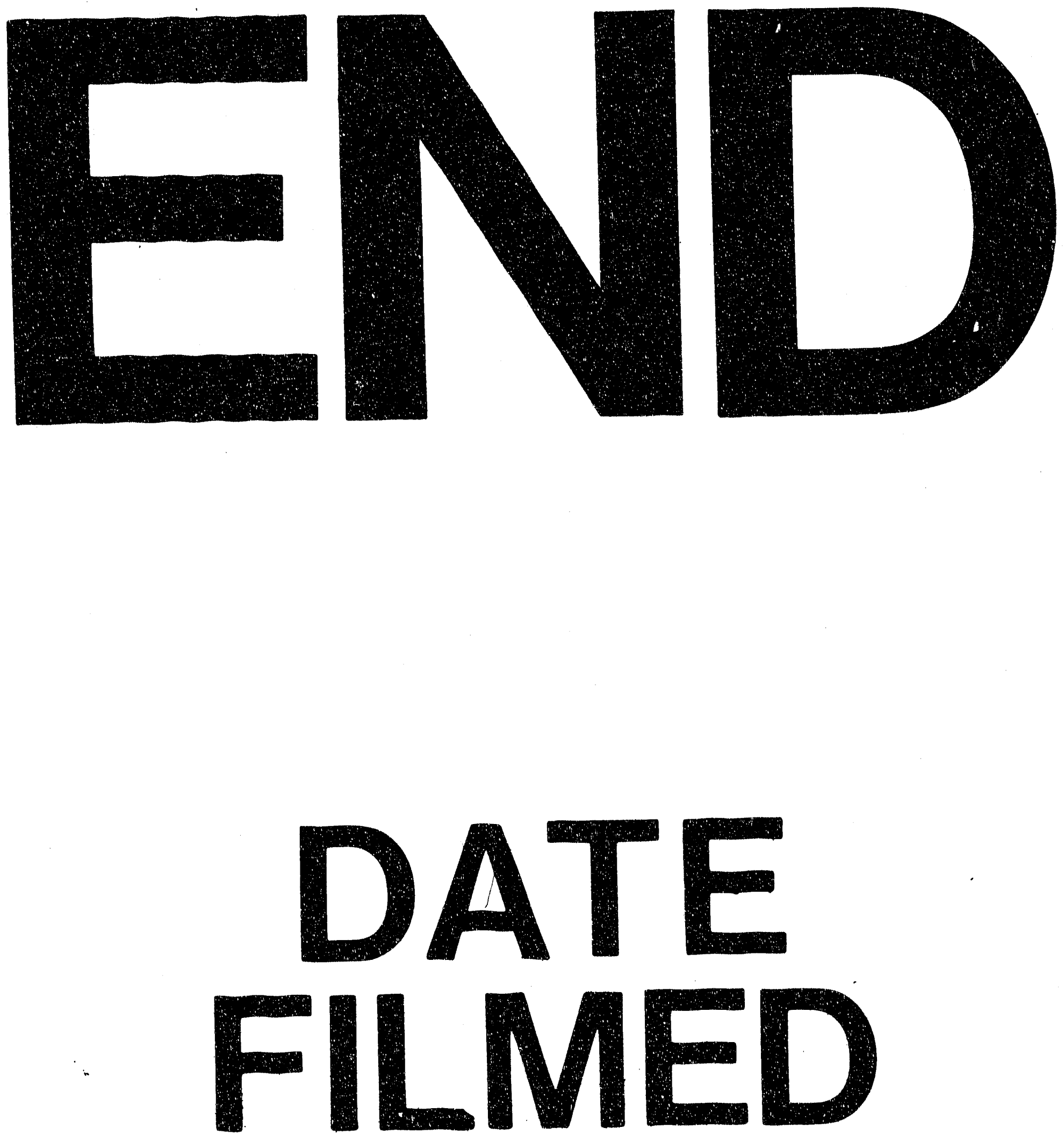

$\underline{=}$

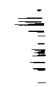

$\Rightarrow$

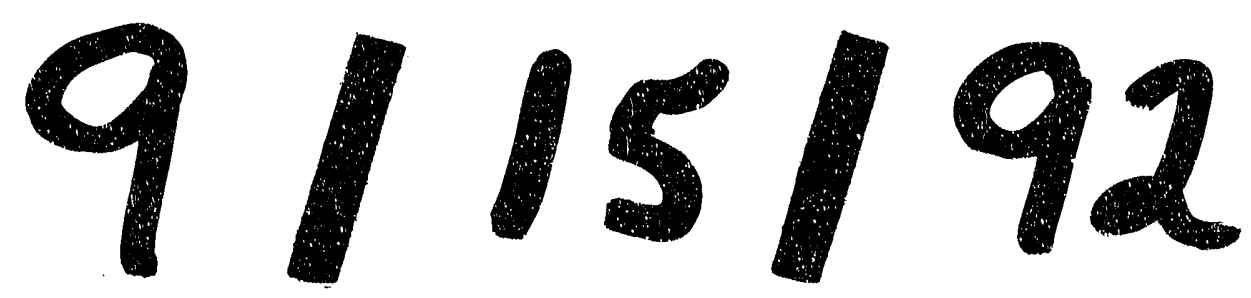


